\title{
Organic Crystals for THz Photonics
}

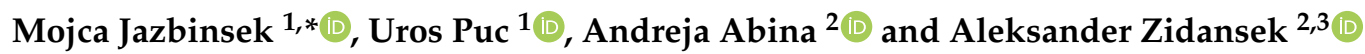 \\ 1 School of Engineering, Zurich University of Applied Sciences, CH-8401 Winterthur, Switzerland; \\ uros.puc@zhaw.ch \\ 2 Jožef Stefan International Postgraduate School, SI-1000 Ljubljana, Slovenia; andreja.abina@mps.si (A.A.); \\ aleksander.zidansek@mps.si (A.Z.) \\ 3 Jožef Stefan Institute, Ljubljana and Department of Physics, University of Maribor, SI-2000 Maribor, Slovenia \\ * Correspondence: mojca.jazbinsek@zhaw.ch; Tel.: +41-58-934-7772
}

Received: 29 January 2019; Accepted: 23 February 2019; Published: 1 March 2019

\begin{abstract}
Organic crystals with second-order optical nonlinearity feature very high and ultra-fast optical nonlinearities and are therefore attractive for various photonics applications. During the last decade, they have been found particularly attractive for terahertz $(\mathrm{THz})$ photonics. This is mainly due to the very intense and ultra-broadband THz-wave generation possible with these crystals. We review recent progress and challenges in the development of organic crystalline materials for THz-wave generation and detection applications. We discuss their structure, intrinsic properties, and advantages compared to inorganic alternatives. The characteristic properties of the most widely employed organic crystals at present, such as DAST, DSTMS, OH1, HMQ-TMS, and BNA are analyzed and compared. We summarize the most important principles for THz-wave generation and detection, as well as organic $\mathrm{THz}$-system configurations based on either difference-frequency generation or optical rectification. In addition, we give state-of-the-art examples of very intense and ultra-broadband $\mathrm{THz}$ systems that rely on organic crystals. Finally, we present some recent breakthrough demonstrations in nonlinear $\mathrm{THz}$ photonics enabled by very intense organic crystalline $\mathrm{THz}$ sources, as well as examples of $\mathrm{THz}$ spectroscopy and $\mathrm{THz}$ imaging using organic crystals as $\mathrm{THz}$ sources for various scientific and technological applications.
\end{abstract}

Keywords: organic nonlinear optics; organic crystals; terahertz waves; electro-optics; differencefrequency generation; optical rectification; THz spectroscopy; THz imaging; DAST; DSTMS; OH1; HMQ-TMS; BNA

\section{Introduction}

Terahertz $(\mathrm{THz})$ waves have recently gained a considerable interest due to a wide variety of interesting applications ranging from fundamental scientific investigations to various industrial implementations. For example, $\mathrm{THz}$ waves are used to study fundamental carrier dynamics phenomena and phonon/vibrational modes of various materials systems [1] and even of single molecules [2]. On the other hand, there is a wide interest in $\mathrm{THz}$ imaging and spectroscopy for industrial materials testing, security, medical applications, food, construction, and the pharmaceutical industry [1]. This development has been made possible by the wide variety of $\mathrm{THz}$ sources that have become available during the last few decades, such as quantum cascade lasers, Gunn lasers, semiconductor antennas, as well as sources that are based on nonlinear optical (NLO) effects. While many of these sources cover only a limited $\mathrm{THz}$ range or can emit at particular $\mathrm{THz}$ frequencies only, the NLO-based sources are of particular interest because they can cover the complete $\mathrm{THz}$ frequency band (frequencies from $0.1 \mathrm{THz}$ to beyond $10 \mathrm{THz}$ ). The NLO-based sources are either based on the NLO process of difference frequency generation or optical rectification. The NLO materials' requirements for both of these types are similar; they need to have a high second-order NLO 
susceptibility and should possibly satisfy the phase-matching condition between the fundamental optical pump waves and the generated $\mathrm{THz}$ waves. For this purpose, mainly three different material systems are considered, semiconducting NLO crystals such as zinc telluride (ZnTe) or gallium phosphide $(\mathrm{GaP})$, the conventional inorganic NLO crystals such as lithium niobate $\left(\mathrm{LiNbO}_{3}\right)$ and organic NLO materials.

The purpose of this review is to discuss the progress in the development of $\mathrm{THz}$ sources and applications based on organic NLO crystals. Section 2 presents the origins of NLO response in organic materials, the intrinsic differences between organic and inorganic NLO materials, and the relations between the relevant microscopic and macroscopic properties of organic NLO crystals. We present and compare the linear and the nonlinear optical properties of some of the presently most widely used organic crystals for THz photonics, including DAST, DSTMS, OH1, HMQ-TMS, and BNA. Section 3 presents theoretical fundamentals of $\mathrm{THz}$-wave generation via difference-frequency generation and via optical rectification. We summarize $\mathrm{THz}$ incoherent and coherent detection techniques that are most widely employed in combination with organic $\mathrm{THz}$ sources. The important phase-matching requirements for efficient THz-wave generation and detection based on NLO principles are also discussed. We compare $\mathrm{THz}$ refractive indices and $\mathrm{THz}$ absorption properties of most widely used organic crystals in relation to their phase-matching characteristics. Section 4 presents some of the most successful examples of organic-based THz sources in terms of power or bandwidth, both the narrowband tunable organic $\mathrm{THz}$ sources based on difference-frequency generation and broadband $\mathrm{THz}$ sources based on optical rectification. Section 5 summarizes various photonics application demonstrations based on organic crystalline $\mathrm{THz}$ sources. These are for example important fundamental demonstrations in the exciting new field of nonlinear $\mathrm{THz}$ photonics, as well as various non-destructive $\mathrm{THz}$ spectroscopy and $\mathrm{THz}$ imaging approaches, interesting for a variety of future scientific and industrial applications.

\section{Second-Order Organic Nonlinear Optical (NLO) Crystals}

The intrinsic NLO response of an organic material depends on the core molecular structure and microscopic nonlinearity of the constituting NLO molecules, their orientational distribution in a particular macroscopic system and the interactions between the molecules. For the particular NLO system configuration such as e.g., for THz-wave generation, the orientation of the molecules relative to the interacting electromagnetic waves and the macroscopic dimensions are essential. In this section, we discuss the involved physical processes and the state-of-the-art material parameters achievable in NLO organic crystals.

\subsection{NLO Effects in Organic Materials}

NLO organic materials are based on the nonlinearity of the constituting molecules. An electric field $\mathbf{E}$ induces a charge redistribution in an organic molecule. This induces a dipole moment $\mathbf{p}$ (in addition to the permanent dipole moment $\mu$ of a molecule), which is most commonly described by the power series as a function of the electric field $\mathbf{E}$

$$
p_{i}=\epsilon_{0} \alpha_{i j} E_{j}+\epsilon_{0} \beta_{i j k} E_{j} E_{k}+\epsilon_{0} \gamma_{i j k l} E_{j} E_{k} E_{l}+\ldots,
$$

where the usual Einstein summation convention holds and where $\epsilon_{0}$ is the electric constant, $\alpha_{i j}$ the linear polarizability, $\beta_{i j k}$ the first hyperpolarizability and $\gamma_{i j k l}$ the second hyperpolarizability of the molecule. Note that, in this review, we use, unless explicitly stated, SI units and the above defined power series expansion, which is the same as in Ref. [3]. Note that several different formulations are used for the above equation in both SI and cgs unit systems, so care must be taken when comparing the results from different sources [3-5]. While all molecules exhibit $\alpha_{i j}$ and $\gamma_{i j k l}$, the first hyperpolarizability $\beta_{i j k}$ is responsible for the asymmetric response to an external field and is only possible in asymmetric molecules. For molecules with non-zero $\beta_{i j k}$, the second-order NLO effects such as second-harmonic 
generation, electro-optic effect, optical rectification, and THz-wave generation are possible and are of interest in this review.

The conventional design of organic molecules to achieve a high first hyperpolarizability $\beta_{i j k}$ is based on a $\pi$-conjugated bridge with strong donor and acceptor substituents at the end of the molecule to promote the asymmetric response and has been extensively studied [6-13]. Figure 1a illustrates the simple physical mechanism responsible for the NLO response of an asymmetric molecule $\left(\beta_{i j k} \neq 0\right)$ to an external electric field: the electronic cloud favors the acceptor A over the donor D. As a consequence, the amplitude of the induced dipole moment depends on the polarity of the applied electric field. Note that the NLO response is usually significant only for very strong electric fields $\mathbf{E}$.

(a)

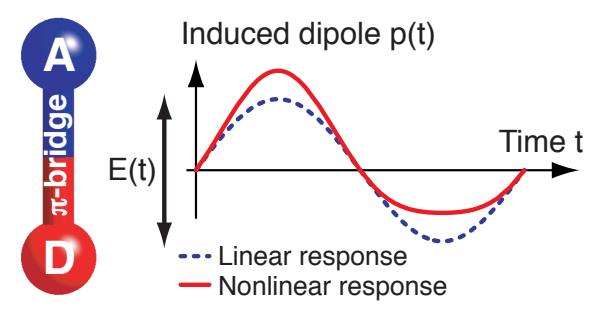

(b)

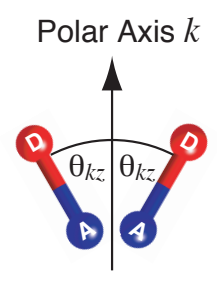

(c) Centrosymmetric structure

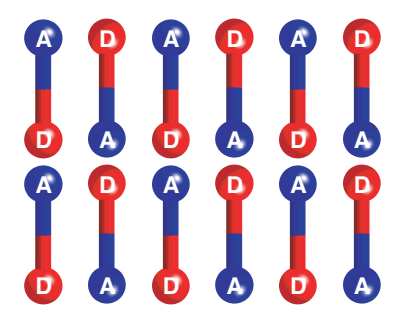

(e) Non-centrosymmetric structure

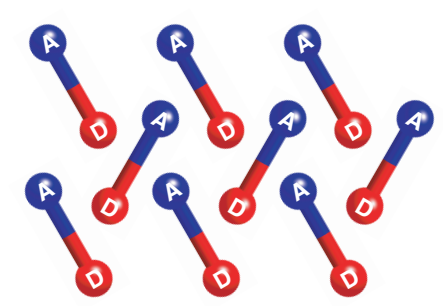

(d) Non-centrosymmetric structure
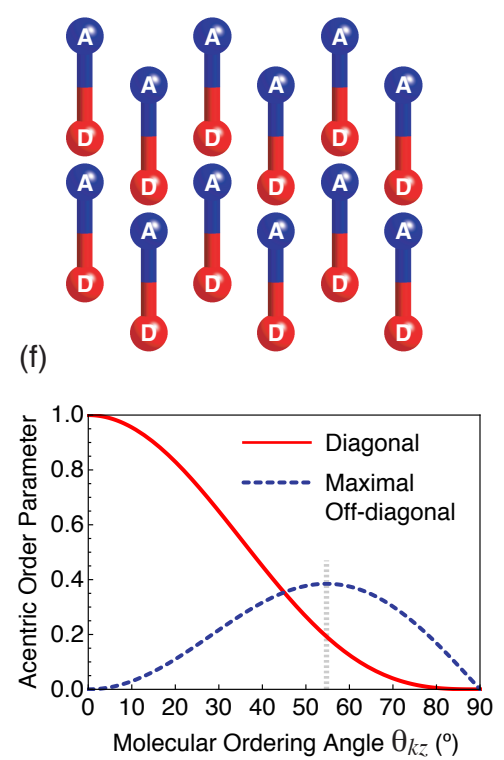

Figure 1. Schematics of: (a) induced dipole moment $p(t)$ of a donor(D)-acceptor(A) substituted $\pi$-conjugated nonlinear optical (NLO) molecule (chromophore) upon application of an external sinusoidal electric field $E(t)$; (b) NLO molecules in the simple oriented-gas model. The molecular-ordering angle $\theta_{k z}$ is the angle between the charge-transfer axis $z$ of the molecule and the polar axis $k$ of the crystal; (c) an example of a centrosymmetric crystal structure with $\theta_{k z}=90^{\circ}$, resulting in zero macroscopic second-order NLO susceptibility $\chi^{(2)}$. Examples of non-centrosymmetric structures with (d) perfectly parallel chromophores with $\theta_{k z}=0^{\circ}$, maximizing the diagonal component of the $\chi^{(2)}$ tensor and with (e) zig-zag-chromophore orientation with $0<\theta_{k z}<90^{\circ}$ with both diagonal and non-diagonal components of $\chi^{(2)}$; (f) the acentric order parameter for the diagonal $\left(\cos ^{3} \theta_{k z}\right)$ and non-diagonal ( $\cos \theta_{k z} \sin ^{2} \theta_{k z}$ ) components of $\chi^{(2)}$ as a function of $\theta_{k z}$ for simplest 1D-chromophores with one dominant first hyperpolarizability component $\beta_{z z z}$.

Many of the state-of-the-art NLO molecules only possess one dominant component of the $\beta_{i j k}$

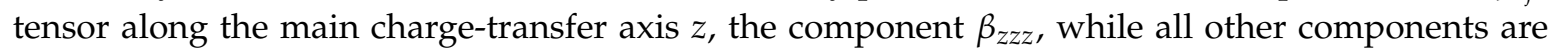
close to zero, which is the so-called 1D-NLO-chromophore approximation [14]. Note that other molecular-design approaches such as the octupolar approach are also being investigated, so that in 
general we need to consider the full tensorial nature of the first hyperpolarizability $\beta_{i j k}$. The first hyperpolarizability of NLO chromophores can be evaluated theoretically using quantum chemical calculations [6,15] or measured experimentally, mostly using the Electric Field Induced Second Harmonic Generation (EFISH) [16] or the hyper-Rayleigh scattering (HRS) technique [17].

The macroscopic NLO material response is in general (also for inorganic materials) described using an analogous power series for the induced material polarization $\mathbf{P}$ as a function of the applied electric field $\mathbf{E}$

$$
P_{i}=\epsilon_{0} \chi_{i j}^{(1)} E_{j}+\epsilon_{0} \chi_{i j k}^{(2)} E_{j} E_{k}+\epsilon_{0} \chi_{i j k l}^{(3)} E_{j} E_{k} E_{l}+\ldots,
$$

where $\chi^{(1)}$ is the linear susceptibility, while $\chi^{(2)}$ and $\chi^{(3)}$ are the nonlinear susceptibilities of the second and the third order, respectively. The susceptibility tensor coefficients are all dependent on the frequency $f$ (or the angular frequency $\omega=2 \pi f$ ) of the involved electric fields $\mathbf{E}$.

As seen in Equation (2), the NLO effects of the second-order, which are of interest in this review, allow interaction of two electric fields $\mathbf{E}\left(\omega_{1}\right)$ and $\mathbf{E}\left(\omega_{2}\right)$ to induce a macroscopic polarization at a combination of their frequencies $\omega_{3}$, which is the source of the generated electric field $\mathbf{E}\left(\omega_{3}\right)$. Figure 2 shows schematic illustrations of two basic second-order NLO processes: sum-frequency generation and difference frequency generation.

(a)

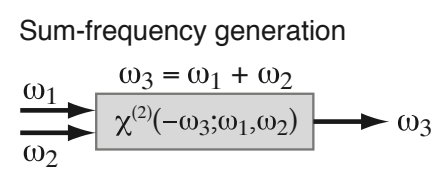

Difference-frequency generation

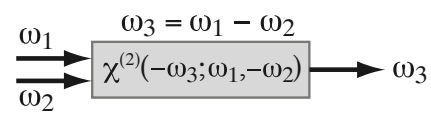

(b) Conservation of photon energy (frequency matching)
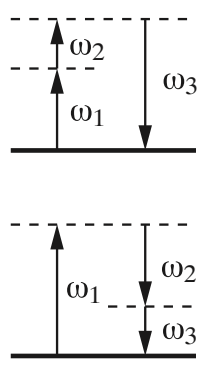

(c) Conservation of photon momentum (phase matching)
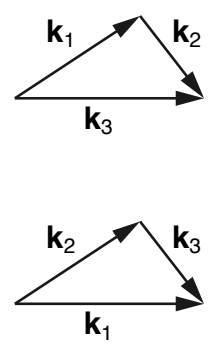

Figure 2. (a) schematics of the two basic parametric second-order NLO processes: sum-frequency generation and difference-frequency generation with $(\mathbf{b})$ the corresponding energy-level diagram and (c) wavevectors $\mathbf{k}_{i}$ that satisfy the phase-matching condition.

In the sum-frequency generation process, the generated frequency is the sum of the two input frequencies $\omega_{3}=\omega_{1}+\omega_{2}$. From the quantum optics point of view, an NLO material allows interactions between photons, so that the total photon energy is conserved $\hbar \omega_{3}=\hbar \omega_{1}+\hbar \omega_{2}$ and the total photon momentum is conserved $\hbar \mathbf{k}_{3}=\hbar \mathbf{k}_{1}+\hbar \mathbf{k}_{2}$ ( $\hbar$ is the reduced Planck constant and $\mathbf{k}_{i}$ the wave vector of the corresponding optical wave). The momentum conservation is in the field of NLO commonly known as the phase-matching condition. In Figure 2c, a general non-collinear phase-matching geometry is presented, while practically often a collinear geometry is preferred, in which all wave vectors are parallel. Any phase mismatch decreases the efficiency of the NLO conversion process. Phase matching requirements relevant for $\mathrm{THz}$-wave generation are discussed in Section 3.4, while the reader is referred to general books on NLO for phase-matching approaches for other processes (see e.g., Refs. [18-20]). The schematic energy-level diagram in Figure $2 b$ illustrates the photon energy conservation with the electronic ground state indicated by the solid line and the virtual states indicated by the dashed lines. The corresponding $\chi^{(2)}$ NLO susceptibility depends on the frequencies of the involved fields, depending on how close these virtual levels are to the real electronic levels. In case one of the virtual levels is close to a real level, the NLO susceptibility is said to be resonantly enhanced. The frequency dependence of the NLO susceptibility for sum-frequency generation is here denoted as $\chi^{(2)}\left(-\omega_{3} ; \omega_{1}, \omega_{2}\right)$, where $\omega_{3}$ is the frequency of the generated electric field. This description holds for the so-called parametric NLO processes, for which the photon energy is conserved and there is no electronic transition within the material involved. For non-parametric 
processes such as, e.g., the two photon-absorption, the imaginary part of the NLO susceptibility should also be considered [19]. A special case of sum-frequency generation is the well-known second-harmonic generation process with $\omega_{1}=\omega_{2}=\omega$, where the generated frequency is twice the pump optical frequency $\omega_{3}=2 \omega$ and only a single-frequency pump optical field is required.

The difference-frequency generation process, also called an optical parametric amplification process, in which generated frequency $\omega_{3}$ presents the difference of the pump frequencies $\omega_{1}-\omega_{2}$, can be explained by the absorption of the first photon at $\omega_{1}$ to a virtual state and then a two-photon emission process, stimulated by the presence of the field at $\omega_{2}$ (see Figure $2 \mathrm{~b}$ ). The field at $\omega_{2}$ can be also spontaneously generated; in this case, the process is referred to as the optical parametric generation. An appropriate optical resonator can be added to an optical parametric generator to enhance the emission; this case is referred to as the optical parametric oscillator.

A special case of difference-frequency generation is the optical rectification process, for which $\omega_{1} \approx \omega_{2} \approx \omega$ and the generated field is a (quasi-)static field at zero (or close to zero) frequency $\omega_{3} \approx 0$. THz-wave generation by difference-frequency generation or optical rectification results in a field at the THz frequency $\omega_{3} \ll \omega_{1}, \omega_{2}$ as discussed in more detail in Section 3 .

For THz-wave generation and electro-optic detection, the relevant processes involve static or quasi-static fields (including THz fields), i.e., oscillating at frequencies much lower than the optical frequencies. The electro-optic effect is most often described as a field-induced change of the refractive index $n$ at $\omega$ as

$$
\Delta\left(\frac{1}{n^{2}}\right)_{i j}=r_{i j k} E_{k}
$$

where the usual summation convention holds and where $r_{i j k}$ is the electro-optic tensor and can be related to the second-order susceptibility as

$$
r_{i j k}(\omega)=-\frac{2 \chi_{i j k}^{(2)}(-\omega ; \omega, 0)}{n_{i}^{2}(\omega) n_{j}^{2}(\omega)},
$$

where $n_{i}(\omega)$ are the frequency-dependent refractive indices along the corresponding dielectric axis $i$.

The linear susceptibility tensor $\chi^{(1)}$ and the related relative permittivity tensor $\epsilon=\chi^{(1)}+1$ describe the common linear polarization response, which depends on the frequency of the applied field $\mathbf{E}(\omega)$. Figure 3 schematically shows the frequency dependence of the relative permittivity $\epsilon(\omega)$. The relative permittivity at low frequencies $\epsilon^{T}$ (zero-stress relative permittivity) contains different contributions from the material polarization response: from acoustic phonon vibrations $\epsilon^{a}$, from optical phonon vibrations $\epsilon^{o}$, which also includes molecular vibrations in organic materials, and from electronic vibrations $\epsilon^{e}$

$$
\epsilon^{T}=\epsilon^{a}+\epsilon^{o}+\epsilon^{e}=\epsilon^{a}+\epsilon^{S}
$$

where $\epsilon^{S}=\epsilon^{o}+\epsilon^{e}$ is the clamped (zero-strain) relative permittivity and $\epsilon^{e}$ determines the refractive index in the optical range as $\epsilon^{e}=n^{2}$. Analogously, the nonlinear susceptibilities $\chi^{(2)}$ and $\chi^{(3)}$ also contain contributions from different possible material polarization responses $[6,21,22]$. The electrons can follow the fastest electric-field oscillations, therefore the electronic polarizability contributes to the linear and the nonlinear susceptibility up until the band-to-band transitions (electronic excitations). The optical phonon vibrations and the molecular vibrations contribute to the polarization response below about the $\mathrm{THz} /$ far-infrared range. The acoustic lattice vibrations can only follow relatively slow electric field oscillations below about the $\mathrm{MHz}$ range.

Due to the different origin of the polarizability in organic and inorganic materials, the frequency dispersion of $\chi^{(i)}(i=1,2$ or 3$)$ is essentially different, which is important for applications. For example, for the most well-known organic crystal DAST (4- $N, N$-dimethylamino- $4^{\prime}-N^{\prime}$-methyl-stilbazolium tosylate), the optical refractive index along the polar axis $a$ is about 2.1 and in the THz range it is about 2.3. The square root $\sqrt{\epsilon^{T}}$ is also about 2.3 [22,23], which points to the very low dispersion 
from the low to the optical frequencies. For the most well-known inorganic $\mathrm{NLO}$ crystal $\mathrm{LiNbO}_{3}$, the optical refractive index along the polar $c$ axis is about 2.2 and in the $\mathrm{THz}$ range it is about 5 , which points to the much larger contribution of lattice vibrations to the polarizability of this crystal. This is also observed in the dispersion of the NLO susceptibility $\chi^{(2)}$ : while the electro-optic coefficient (involving quasi-static fields) is of the same order of magnitude for DAST $\left(r_{111}=48 \mathrm{pm} / \mathrm{V}\right.$ at $1535 \mathrm{~nm}$ ) and $\mathrm{LiNbO}_{3}\left(r_{333}=30 \mathrm{pm} / \mathrm{V}\right.$ at $\left.1064 \mathrm{~nm}\right)$, the difference is much larger for the NLO coefficient for second-harmonic generation, involving only the optical fields $\left(d_{111}=290 \mathrm{pm} / \mathrm{V}\right.$ at $1535 \mathrm{~nm}$ for DAST and $d_{333}=34 \mathrm{pm} / \mathrm{V}$ at $1064 \mathrm{~nm}$ for $\left.\mathrm{LiNbO}_{3}\right)[21,22,24]$. This shows that the origin of the linear and the nonlinear optical susceptibility in organic materials is essentially electronic and therefore extremely fast (femtoseconds). This is an important advantage for high-speed electro-optic modulation $[6,25,26]$ and also for $\mathrm{THz}$-wave generation and detection: due to the approximate matching of the $\mathrm{THz}$ and the optical refractive index, it is much easier to achieve the phase matching needed for highly efficient response, as discussed in Section 3.4.

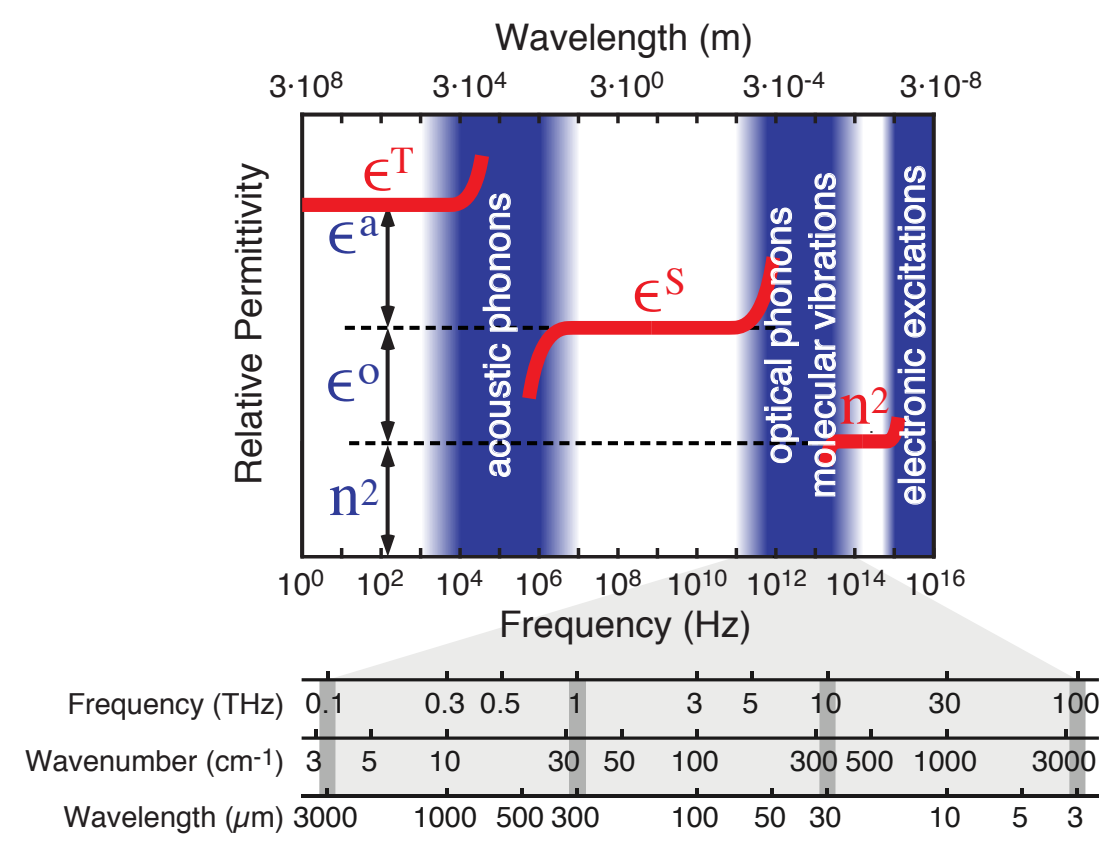

Figure 3. Schematics of the material linear polarization response as a function of frequency of the external electric field. For organic materials, the contributions of $\epsilon^{o}$ and $\epsilon^{a}$ to the static relative permittivity $\epsilon^{T}$ are much less important compared to inorganic materials, which is an important advantage for high-speed electro-optic modulation and THz-photonics applications (see text). The scale below shows the THz frequency range in frequency $f$, wavelength $\lambda$, and spectroscopic wavenumber $1 / \lambda$.

\subsection{Organic Single Crystalline Materials}

To achieve a macroscopic second-order NLO response in organic materials with non-zero $\chi^{(2)}$, the constituting asymmetric NLO chromophores have to be distributed non-centrosymmetrically. This is by far not trivial because these molecules usually possess a high permanent dipole moment and they tend to orient antiparallel, as illustrated in Figure 1c, which cancels the macroscopic $\chi^{(2)}$ response. There have been many possibilities proposed to achieve a macroscopic non-centrosymmetric order of NLO molecules. Most commonly, a non-centrosymmetric order is achieved by incorporating NLO chromophores in a polymer matrix and then pole the material by applying a very strong static electric field (just below the electric breakdown) above the glass transition temperature to align these molecules, based on their relatively high permanent dipole moment $\mu$. The advantage of this approach is that it is in principle applicable for all chromophores with a permanent dipole moment, including those with the highest first hyperpolarizabilities $\beta$ [6]. On the other hand, it is 
extremely challenging to achieve a desired long-term orientational stability of the non-centrosymmetric order at room temperature [6]. For $\mathrm{THz}$ photonics applications, the achievable material thickness of polymer-based materials is also very limited [27]. For highly-efficient THz-wave generation, the thickness of the $\mathrm{THz}$ generation material (organic or inorganic) in the range of $0.1-1 \mathrm{~mm}$ is required [28]. Another possibility to obtain an efficient macroscopic second-order active NLO organic material is by crystallization, offering the highest possible chromophore density, the best possible long-term orientational stability and sufficient sample thickness $(\sim 0.1-1 \mathrm{~mm}$ range), which is the approach discussed in this review. Additional to the much superior temporal stability of crystals compared to poled polymers, their photochemical stability may be better by several orders of magnitude [29], which is particularly important for THz-wave applications, where high optical power densities are used and therefore a high damage threshold is required. On the other hand, only a very small ratio of known chromophores can crystallize in a non-centrosymmetric crystalline space group and there seems to be a limit in the conjugation length (i.e., the first hyperpolarizability strength $\beta$ ) that can still lead to a non-centrosymmetric alignment $[15,30]$. The chromophores, besides having a large $\beta$, need to be usually specially designed to promote a non-centrosymmetric alignment $[15,30]$. In addition, even for the chromophores that naturally self assemble into a non-centrosymmetric structure, the growth of high optical quality crystals large enough and with desired morphology may be too challenging to allow for practical applications. For all these reasons, although there are endless possibilities for molecular engineering to obtain organic NLO crystals, there have been only a few identified that lead to crystals that can be potentially used for practical NLO applications and some of these are presently commercially available. The commercial availability of these crystals has helped to considerably expand the research possibilities particularly for THz-wave generation with organic NLO crystals during the last couple of years, which has additionally motivated the continued research and development of novel and optimized organic NLO crystals for THz photonics applications.

The relation between the macroscopic and the molecular NLO coefficients is complex because the interactions between the neighboring molecules influence the first hyperpolarizability $\beta$ in the crystalline form $[11,31,32]$. However, often the macroscopic second-order nonlinearities of organic materials can be reasonably well explained by the nonlinearities of the constituent molecules by the so-called oriented-gas model [14]. This relation is in particular simple for the chromophores in the 1D approximation, for which only one dominant tensor element $\beta=\beta_{z z z}$ can be considered, which is the case of most state-of-the-art organic NLO crystals. For example, the maximal diagonal element for the NLO susceptibility along the polar axis $k$ of the crystal can be expressed as

$$
\chi_{k k k}^{(2)}=N f_{\text {local }} \beta_{k k k}^{\text {eff }}=N f_{\text {local }}\left\langle\cos ^{3} \theta_{k z}\right\rangle \beta_{z z z}
$$

The above relation reveals the most important contributions to the NLO effect in organic materials: the chromophore number density $N$, the local-field factor $f_{\text {local }}$, the molecular nonlinearity $\beta_{z z z}$ along the main charge-transfer axis of the chromophore, and the orientational factor, the so-called acentric order parameter $\left\langle\cos ^{3} \theta_{k z}\right\rangle$, which is averaged over all molecules in a macroscopic system. For crystalline materials, the angle $\theta_{k z}$ is the angle between the main charge transfer axis $z$ of the chromophores and the polar axis $k$ of the crystal, as illustrated in Figure $1 b$. Note that, for simplicity and practical relevance, we define here the polar axis $k$ along the effective charge-transfer direction of the crystal, which may be, in low symmetry crystals (monoclinic and triclinic) slightly off (for highly nonlinear crystals, not more than a few degrees), the real polar axis defined by the effective permanent dipole moment of the crystal.

The effective first hyperpolarizability coefficients $\beta_{i j k}^{\text {eff }}$ in the crystal can be introduced to consider the first hyperpolarizability tensor components $\beta_{x y z}$ of all single chromophores in the crystalline unit cell projected to a suitable Cartesian coordinate system of the crystal based on the oriented-gas model as $[14,15]$

$$
\beta_{i j k}^{\text {eff }}=\frac{1}{n} \sum_{s} \sum_{x y z} \cos \left(\theta_{i x}^{s}\right) \cos \left(\theta_{j y}^{s}\right) \cos \left(\theta_{k z}^{s}\right) \beta_{x y z}
$$


where $n$ denotes the number of chromophores in the unit cell, $s$ the site in the unit cell and $\theta_{i m}^{s}$ are the angles between the dielectric axes $i(i=1,2,3)$ of the crystal and the molecular axis $m(m=x, y, z)$ of the chromophore. The macroscopic second-order susceptibility tensor components can be then evaluated as

$$
\chi_{i j k}^{(2)}=N f_{\text {local }} \beta_{i j k}^{\text {eff }}
$$

Note that both the effective first hyperpolarizability components $\beta_{i j k}^{\text {eff }}$ as well as the local-field factors $f_{\text {local }}$ strongly depend on the frequencies of the interacting electric fields.

The effective first hyperpolarizability components vanish in case of a centrosymmetric crystalline arrangement $\left(\beta_{i j k}^{\text {eff }}=0\right)$ like the alignment illustrated in Figure 1c. For perfectly parallel chromophore alignment along the $k$-axis as illustrated in Figure $1 \mathrm{~d}$ and 1D chromophore approximation, the diagonal component along the polar axis is maximal $\beta_{k k k}^{\text {eff }}=\beta_{z z z}$ and all other tensor components of $\beta_{i j k}^{\text {eff }}$ are zero. For an orientation with $0<\theta_{k z}<90^{\circ}$ and in 1D chromophore orientation as illustrated in Figure 1e, the maximal diagonal effective first hyperpolarizability component is $\beta_{k k k}^{\text {eff }}=\beta_{z z z} \cos ^{3} \theta_{k z}$ and the maximal off-diagonal effective first hyperpolarizability component is $\beta_{i i k}^{\text {eff }}=\beta_{z z z} \cos \theta_{k z} \sin ^{2} \theta_{k z}$. The dependence of maximal diagonal and maximal off-diagonal components of $\beta_{i j k}^{\text {eff }}$ are illustrated in Figure 1f. Note that in case the chromophores are additionally distributed in the azimuthal angle around the polar axis, another projection needs to be considered for different off-diagonal effective first hyperpolarizability components [14]. As seen in Figure 1f, to maximize the diagonal NLO susceptibility $\chi_{k k k}^{(2)}$ along the polar axis $k$, the projection angles $\theta_{k z}$ should be close to zero, i.e., the charge transfer axes of the molecules should be close to parallel. To maximize the off-diagonal susceptibility elements $\chi_{i k k}^{(2)}$ the molecules should make an angle of about $54.7^{\circ}$ with respect to the polar axis [14]. For electro-optic and $\mathrm{THz}$ photonics applications, for the majority of cases, the large diagonal NLO coefficient is desired and therefore the molecular orientation with small or zero $\theta_{k z}$ is optimal.

The dispersion of the macroscopic NLO susceptibilities is usually modeled using Equation (8), by assuming a simple two-level model [33] for the dispersion of first hyperpolarizabilities $\beta_{z z z}$ and local field factors $f_{\text {local }}[8,9]$. The dispersion depends on the frequencies of all involved interacting electric fields, therefore the NLO susceptibilities are different for different NLO processes. For the linear electro-optic effect, the dispersion of the dominant first hyperpolarizability component is in the two-level model given by

$$
\beta_{z z z}(-\omega ; \omega, 0)=\frac{\omega_{e g}^{2}\left(3 \omega_{e g}^{2}-\omega^{2}\right)}{3\left(\omega_{e g}^{2}-\omega^{2}\right)^{2}} \beta_{0}
$$

and for the second-harmonic generation by

$$
\beta_{z z z}(-2 \omega ; \omega, \omega)=\frac{\omega_{e g}^{4}}{\left(\omega_{e g}^{2}-4 \omega^{2}\right)\left(\omega_{e g}^{2}-\omega^{2}\right)} \beta_{0},
$$

where $\omega_{\text {eg }}$ denotes the resonant angular frequency of the electronic transition from the ground $(g)$ to the excited (e) state and a dispersion-free first hyperpolarizability $\beta_{0}$ presents the first hyperpolarizability extrapolated to infinite wavelengths $(\omega \rightarrow 0)$, away from electronic resonances. The simple two-level model describes well the dispersion away from electronic resonances. More sophisticated models are available for modeling the dispersion closer or at electronic resonances such as the homogeneously damped two-level model [34,35], the homogeneously broadened vibronic model $[34,36]$ and the inhomogeneously broadened vibronic model [36].

The dispersion-free first hyperpolarizability $\beta_{0}$ is a very useful parameter when comparing the nonlinearity of molecules with different resonant frequencies $\omega_{e g}$. In the conventional two-level model [33], the dispersion-free first hyperpolarizability $\beta_{0}$ depends on $\omega_{e g}$, the difference $\Delta \mu=\mu_{e}-\mu_{g}$ 
between the excited $\mu_{e}$ and the ground-state $\mu_{g}$ dipole moments and the transition dipole moment $\mu_{\text {eg }}$ as

$$
\beta_{0}=\frac{3}{2 \epsilon_{0} \hbar^{2}} \frac{\Delta \mu \mu_{e g}^{2}}{\omega_{e g}^{2}} .
$$

The inverse-square dependence of $\beta_{0}$ on $\omega_{\text {eg }}$ is often referred to as the nonlinearity-transparency trade-off: the chromophores with a red-shifted absorption maximum (lower transmission in the visible wavelength range, lower $\omega_{\text {eg }}$ ) typically present a higher $\beta_{0}$. This is strongly related to the best phase-matching range for $\mathrm{THz}$-wave generation with state-of-the-art organic materials, as discussed later on.

The macroscopic second-order susceptibility coefficients $\chi_{i j k}^{(2)}$ can be evaluated by the conventional Maker-fringe technique and electro-optic measurements $[6,9,37,38]$. For these measurements, high optical quality plane-parallel samples with polished surfaces and possibly with different orientations are required and the refractive index dispersion should be also measured with a relatively high precision (see an example in Ref. [39]), which is very time-consuming. For newly developed organic NLO crystals, simpler screening methods are therefore employed to evaluate their potential for NLO applications.

A relatively simple Kurtz and Perry second-harmonic generation powder test can be performed to screen the new materials [40]. The test can be done qualitatively only to determine if the material is non-centrosymmetric and estimate the order of magnitude for the $\chi^{(2)}$-effects, but also more qualitatively including the measurements of the second-harmonic generation efficiency as a function of powder size and as a function of pump wavelength, which may indicate a possible phase-matching enhancement [40]. For highly nonlinear organic crystals that typically present a red-shifted absorption edge in the visible (above ca. $600 \mathrm{~nm}$, see the following section), the coherence length for second-harmonic generation is relatively short (in the order of $1 \mu \mathrm{m}$ ), much smaller than the typical powder size, which gives then a relatively good estimate for the macroscopic susceptibility based on the powder test (see, e.g., Figure 15 in Ref. [15]).

Another possibility to estimate the $\chi^{(2)}$-potential of novel materials is to evaluate first the molecular nonlinearities $\beta_{x y z}$ by quantum chemical calculations, determine the crystal structure by $X$-ray diffraction and then use the above-mentioned oriented-gas model according to Equations (7) and (8). Because of the additional influence of intermolecular interactions and local field corrections, the theoretical approximation with the effective first hyperpolarizability tensor $\beta_{i j k}^{\text {eff }}$ only roughly explains the actually measured NLO and electro-optic coefficients but is still well suited to compare different materials and their potential for NLO applications [15].

More recently, several groups have used newly developed organic NLO crystals directly for THz-wave generation experiments and compared the generated $\mathrm{THz}$ fields with some standard material such as ZnTe; some examples are mentioned in Section 4.2. This method is also relatively convenient in case an appropriate $\mathrm{THz}$ system is available because the basic requirements for the sample quality for THz-wave generation are relatively low compared to the requirements for the optical characterization. This is because the (surface) quality requirements scale with the wavelength, which is about two orders of magnitude larger for THz compared to the optical waves.

\subsection{Optical and NLO Properties of Organic Crystals}

Figure 4 shows the crystal structures and the dominant sample morphology of some of the most well investigated organic NLO crystals for THz-wave generation during the last decade: the stilbazolium salts DAST and DSTMS (4-N,N-dimethylamino- $4^{\prime}-N^{\prime}$-methyl-stilbazolium 2,4,6-trimethylbenzenesulfonate), the non-ionic configurationally locked polyene crystal $\mathrm{OH} 1$ (2-(3-(4-hydroxystyryl)-5,5-dimethylcyclohex-2-enylidene)malononitrile) and the quinolinium salt HMQ-TMS (2-(4-hydroxy-3-methoxystyryl)-1-methylquinolinium 2,4,6-trimethylbenzenesulfonate). The relevant crystallographic properties and the microscopic/macroscopic NLO properties of 
these crystals are listed in Table 1, which includes another widely investigated material BNA (N-Benzyl-2-methyl-4-nitroaniline). Examples of refractive index dispersion and electro-optic coefficient dispersion in the optical range are shown in Figures 5 and 6. In the following subsections, we discuss these properties in more detail for the selected ionic and non-ionic organic NLO crystals.

(a) DAST
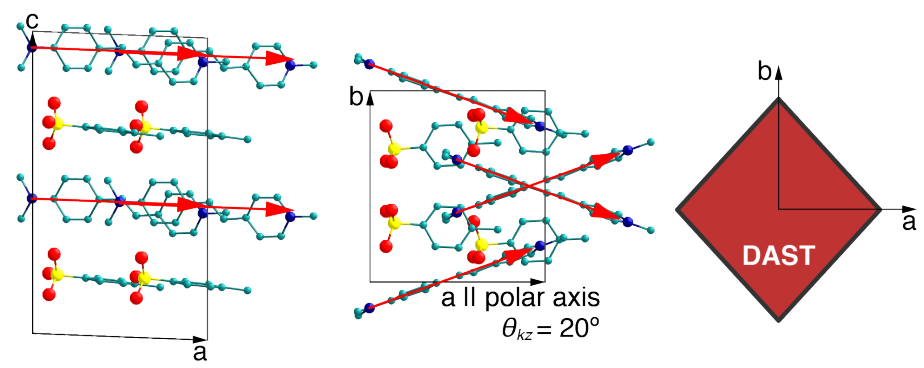

(b)

DSTMS
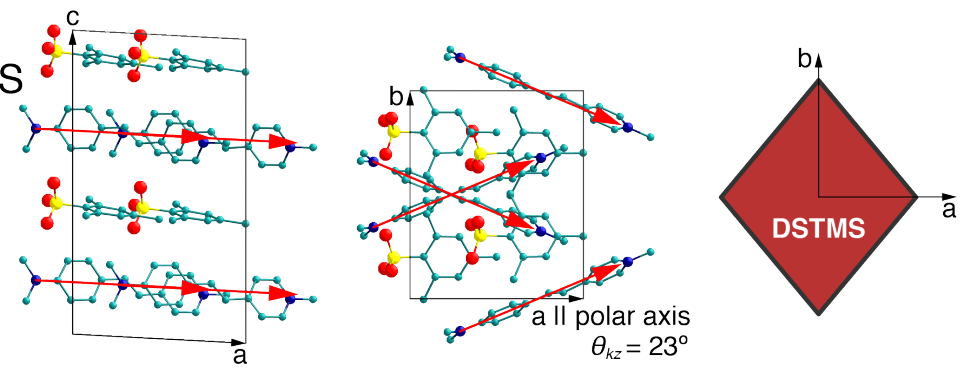

(c)

$\mathrm{OH} 1$
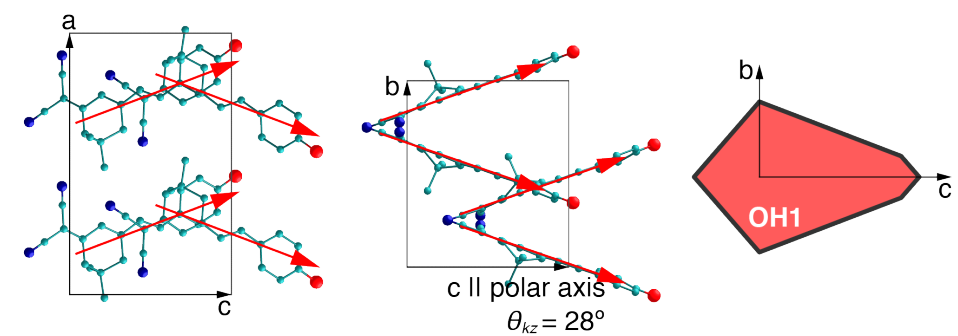

(d)
HMQ-TMS
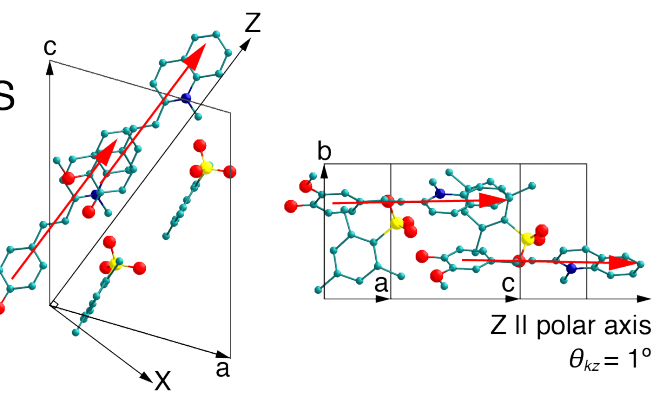

ac-plane

(cleaved)

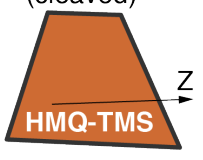

Figure 4. Crystal structures and the dominant sample morphology of some of the most well investigated organic NLO crystals for THz-wave generation during the last decade: the stilbazolium salts DAST (a) [22,41-43] and DSTMS (b) [32,44], the non-ionic configurationally locked polyene crystal OH1 (c) [45,46] and the quinolinium salt HMQ-TMS (d) [47]. The structures are available at the Cambridge Crystallographic Centre (CCDC) for the corresponding publications. The red arrows indicate the direction of the main charge-transfer axis of the NLO chromophores. The typical plate-like sample morphologies used in THz applications (as-grown for DAST, DSTMS, OH1 and cleaved for HMQ-TMS) have been drawn based on the information given in the corresponding references.

\subsubsection{Ionic Organic Crystal DAST}

DAST is the most well known and widely investigated organic electro-optic crystal. DAST was first reported already in 1989 by [41,42] and is still being recognized as one of the best state-of-the-art 
organic NLO crystals. High optical quality and large size DAST crystals can be grown from methanol solution by the slow cooling method $[43,48]$. DAST crystals have high second-order NLO and electro-optic coefficients, being respectively ten times and twice as large as those of the inorganic standard $\mathrm{LiNbO}_{3}$. Due to the relatively low zero-stress relative permittivity compared to inorganic alternatives, as discussed in the previous section, DAST is highly attractive for high-speed electro-optic applications and broadband THz-wave generation [22]. DAST is an organic salt that consists of a positively charged stilbazolium cation and a negatively charged tosylate anion. The stilbazolium cation is one of the most efficient NLO active chromophores that can crystallize in a non-centrosymmetric structure $[15,30]$. The counter ion tosylate is used to promote the non-centrosymmetric crystallization, but its contribution to the macroscopic nonlinearity is negligible compared to the cation [41,49].

Table 1. Crystallographic symmetry, wavelength of absorption maximum $\lambda_{\text {max }}$ in methanol solution, main first hyperpolarizability component $\beta_{z z z}$ (non-resonant) calculated by quantum-chemical calculations, the angle $\theta_{k z}$ between the main charge-transfer axis $z$ of the chromophores and the polar axis $k$ of the crystal, the non-zero elements of the effective first hyperpolarizability tensor $\beta_{i j k}^{\text {eff }}$ (non-resonant; i.e., Kleinman symmetry valid), and the measured electro-optic coefficients $r_{i j k}$ and nonlinear optical (NLO) coefficients for second-harmonic generation $d_{i j k}(\omega)=\frac{1}{2} \chi^{(2)}(-2 \omega ; \omega, \omega)$ at different wavelengths of some of the most well investigated organic NLO crystals for THz-wave generation. The results for first hyperpolarizabilities are given in cgs units because these are most often reported; $10^{-30}$ esu corresponds to $4.18 \cdot 10^{-40} \mathrm{~m}^{4} / \mathrm{V}$ according to the definition of the first hyperpolarizability in Equation (1).

\begin{tabular}{|c|c|c|c|c|c|c|}
\hline $\begin{array}{l}\text { Crystal Name } \\
\text { [Refs.] }\end{array}$ & $\begin{array}{l}\text { Space Group } \\
\text { (Point Group) }\end{array}$ & $\begin{array}{l}\lambda_{\max } \\
(\mathrm{nm})\end{array}$ & $\begin{array}{l}\beta_{z z z} \\
\left(10^{-30} \mathrm{esu}\right)\end{array}$ & $\begin{array}{l}\theta_{k z} \\
\left({ }^{\circ}\right)\end{array}$ & $\begin{array}{l}\beta_{i j k}^{\text {eff }} \\
\left(10^{-30} \text { esu }\right)\end{array}$ & $\begin{array}{l}d_{i j k}, r_{i j k} \\
(\mathrm{pm} / \mathrm{V})\end{array}$ \\
\hline $\begin{array}{l}\text { DAST } \\
{[22,23,41-43,50,51]}\end{array}$ & $\begin{array}{l}C c \\
(m)\end{array}$ & 475 & 194 & 20 & $\begin{array}{l}\beta_{111}^{\text {eff }}=161 \\
\beta_{221}^{\text {eff }}=21 \\
\beta_{331}^{\text {eff }}=-3.2 \\
\beta_{113}^{\text {eff }}=0.0 \\
\beta_{223}^{\text {eff }}=1.0 \\
\beta_{333}^{\text {eff }}=-0.3\end{array}$ & $\begin{array}{l}d_{111}(1318 \mathrm{~nm})=1010 \\
d_{111}(1542 \mathrm{~nm})=290 \\
d_{111}(1907 \mathrm{~nm})=210 \\
d_{122}(1907 \mathrm{~nm})=32 \\
d_{212}(1907 \mathrm{~nm})=25 \\
r_{111}(720 \mathrm{~nm})=92 \\
r_{111}(1313 \mathrm{~nm})=53 \\
r_{111}(1535 \mathrm{~nm})=47 \\
r_{221}(1535 \mathrm{~nm})=21\end{array}$ \\
\hline $\begin{array}{l}\text { DSTMS } \\
{[32,44,50,52]}\end{array}$ & $\begin{array}{l}\mathrm{Cc} \\
(m)\end{array}$ & 475 & - & 23 & - & $\begin{array}{l}d_{111}(1907 \mathrm{~nm})=214 \\
d_{122}(1907 \mathrm{~nm})=31 \\
d_{212}(1907 \mathrm{~nm})=35\end{array}$ \\
\hline $\begin{array}{l}\text { OH1 } \\
{[39,45,46]}\end{array}$ & $\begin{array}{l}P_{n a 2_{1}} \\
(m m 2)\end{array}$ & 426 & 93 & 28 & $\begin{array}{l}\beta_{333}^{\text {eff }}=63 \\
\beta_{223}^{\text {eff }}=7 \\
\beta_{113}^{\text {eff }}=9\end{array}$ & $\begin{array}{l}d_{333}(1907 \mathrm{~nm})=120 \\
r_{333}(633 \mathrm{~nm})=109 \\
r_{333}(1319 \mathrm{~nm})=52 \\
r_{223}(1319 \mathrm{~nm})=30 \\
r_{113}(1319 \mathrm{~nm})=6.8\end{array}$ \\
\hline $\begin{array}{l}\text { HMQ-TMS } \\
{[47,53,54]}\end{array}$ & $\begin{array}{l}P n \\
(m)\end{array}$ & 439 & 185 & 1 & $\begin{array}{l}\beta_{333}^{\text {eff }}=185 \\
\beta_{223}^{\text {eff }}=0.7 \\
\beta_{113}^{\text {eff }}=-0.8 \\
\beta_{111}^{\text {eff }}=0.7 \\
\beta_{221}^{\text {eff }}=0.3 \\
\beta_{331}^{\text {eff }}=0.0\end{array}$ & $r_{333}(633 \mathrm{~nm}) \gtrsim 50$ \\
\hline $\begin{array}{l}\text { BNA } \\
{[55-59]}\end{array}$ & $\begin{array}{l}P_{n a 2_{1}} \\
(m m 2)\end{array}$ & 383 & - & 33 & - & $\begin{array}{l}d_{333}(1064 \mathrm{~nm})=234 \\
d_{322}(1064 \mathrm{~nm})=15.6 \\
d_{223}(1349 \mathrm{~nm})=4.5\end{array}$ \\
\hline
\end{tabular}


The structure of DAST is shown in Figure 4a. The chromophores are packed with their main charge transfer axis oriented at about $\theta_{k z}=20^{\circ}$ with respect to the polar axis $k=a$, resulting in a high diagonal acentric order parameter of $\cos ^{3} \theta_{k z}=0.83$ (see also Figure 1f), which is close to optimal for the electro-optic and $\mathrm{THz}$ photonics applications. Due to the high diagonal acentric order parameter, DAST crystals are strongly anisotropic with a refractive index difference $n_{1}-n_{2}>0.5$ in the visible and infrared wavelength range $[22,23,48]$. The dispersion of the refractive index is essential for phase-matched THz-wave generation and scales with the absorption band (electronic resonances) of materials according to the general Kramers-Kronig relation. DAST crystals are well suited in particular for applications at around telecommunications wavelengths, with a material absorption that is smaller than $0.1 \mathrm{~mm}^{-1}$ at $1300 \mathrm{~nm}$ and $1550 \mathrm{~nm}$ (see Figure $5 \mathrm{~b}$ ). As discussed later, this range is also best suited as pump wavelength range for THz-wave generation and detection with DAST.

DAST has very high NLO coefficients and electro-optic coefficients, as listed in Table 1 . The highest low-frequency electro-optic tensor components $r_{i j k}$ measured in DAST are shown in Figure 6a. The measured dispersion has been modeled by the two-level model according to Equation (9) and is represented by solid or dashed curves in Figure 6a [23]; the deviation at shorter wavelengths is due to the limits of the simple two-level model close to the absorption edge as discussed below Equation (10). The particular orientation of the NLO stilbazolium chromophores shown in Figure 4a defines the main diagonal and non-diagonal components of the macroscopic NLO susceptibility.
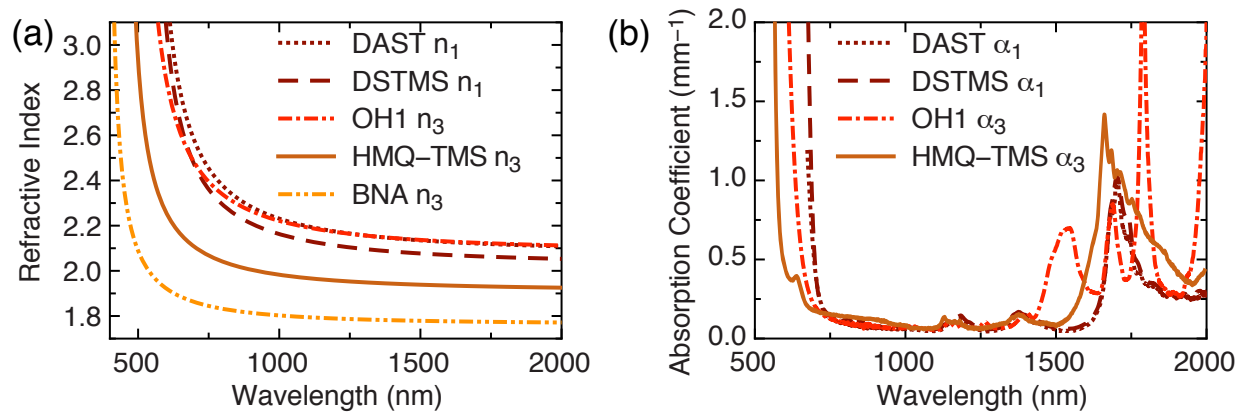

Figure 5. (a) refractive index and (b) absorption coefficient as a function of wavelength for some of the state-of-the-art organic crystals for light polarized along the polar axis of the particular crystal: DAST [22,23], DSTMS [52], OH1 [39], HMQ-TMS [53] and BNA [56].
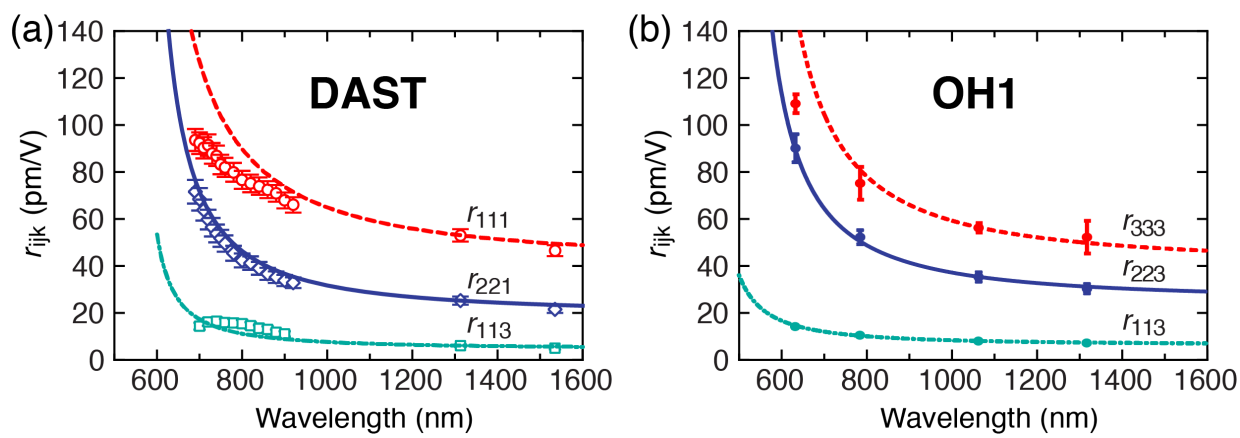

Figure 6. Dispersion of the largest electro-optic coefficients in (a) DAST [22,23] and (b) OH1 [39].

\subsubsection{Alternative Ionic Organic NLO Crystals}

Theoretical evaluations additionally show that the upper limits of NLO susceptibilities in organic crystals have not been reached yet $[11,31]$. This is one of the reasons that the research and development of novel NLO organic crystals is continuing. Another reason for the reinforced development during the last couple of years is a requirement for different optical and $\mathrm{THz}$ properties that would allow applications in different wavelength regimes, as discussed in details in Section 3.4. Several salt-type organic crystals, i.e., with NLO cation and a counter-anion that promotes non-centrosymmetric 
packing, have been identified with similar or even superior NLO properties than DAST [32,49,50,60-65]. For photonics applications, not only high nonlinear and appropriate linear optical properties are required. Many of the novel crystals may be very challenging to grow into suitable samples for applications or even simple characterization. Therefore, it is also important to develop organic NLO crystals with easier crystal growth procedures $[44,66]$ or better crystal processing possibilities. For example, a possibility for cleaving as-grown crystals to produce samples with a desired thickness and optical-quality surfaces is very attractive because the expensive and time-consuming cutting and polishing may not be necessary anymore $[47,67]$. In addition, various crystal engineering approaches are being developed that can lead to crystals with a desired final morphology [68-71]. Two of the most successful organic NLO salt crystals developed during the last decade, considering their properties and growth/processing possibilities as well as reports on applications, are DSTMS and HMQ-TMS, which are discussed in the following subsections.

DSTMS [44] is structurally very similar to DAST: it has the same NLO cation chromophore as DAST, but the counter-anion is slightly modified (from 4-methylbenzenesulfonate to 2,4,6-trimethylbenzenesulfonate). The additional methyl groups on the anion of DSTMS do not considerably affect both the crystal structure and the nonlinear optical properties (see Figure 4 and Table 1). However, DSTMS has an important advantage for the crystal growth because the solubility in methanol is twice as large as in DAST [44]. In addition, the strongest THz phonon absorption peak at about $1 \mathrm{THz}$ is in DSTMS only about half as that of DAST [72]. DSTMS has been employed by several research groups for $\mathrm{THz}$ photonics applications [72-81].

Another ionic crystal HMQ-TMS has a quinolinium-based ionic-cation core structure. The quinolinium derivatives have been recently identified as very attractive for NLO applications, resulting in non-centrosymmetric crystals with a relatively high probability [15]. HMQ-TMS has been first reported in 2013 [47] and has been already employed in different $\mathrm{THz}$ photonics schemes $[53,54,71,82-84]$. The quinolinium chromophores in HMQ-TMS are oriented almost perfectly parallel (see Figure 4d) and therefore the diagonal NLO response is maximized. Although the NLO properties of HMQ-TMS may be comparable or slightly lower compared to DAST (see Table 1 [15,54]), a big advantage of this crystal is a possibility to obtain high optical-quality samples by a simple cleaving method [47]. HMQ-TMS has a blue-shifted absorption edge (see Figure 5) and different optical and THz properties compared to DAST and DSTMS, so it may be preferred for specific applications.

\subsubsection{Non-Ionic Organic NLO Crystals}

Besides organic salt crystals, the non-ionic organic NLO crystals have also gained considerable attention during the last decade for integrated electro-optic applications and $\mathrm{THz}$ photonics. The configurationally locked polyene crystal $\mathrm{OH} 1$ [45] has become very attractive due to the electro-optic coefficients as high as for ionic DAST, combined with a blue-shifted absorption edge and different $\mathrm{THz}$ properties $[39,85]$. $\mathrm{OH} 1$ exhibits a relatively high crystal symmetry (orthorhombic, see Figure 4c) compared to the majority of other highly nonlinear organic crystals (monoclinic or even triclinic), which simplifies the handling and characterization. $\mathrm{OH} 1$ has very good crystal processing possibilities from solution, including high optical quality bulk crystals $[46,68,71]$ and large-area single crystalline thin films on substrates [86]. High-quality OH1 thin films can be also grown by the physical vapor deposition [87]. $\mathrm{OH} 1$ has been found particularly effective for THz-wave applications [85,88-98].

Another non-ionic NLO organic crystal used for THz-wave generation by several groups is BNA (N-Benzyl-2-methyl-4-nitroaniline) [55]. BNA is based on the nitroaniline derivatives developed in the early days of organic NLO research [99]. Because of the yellow color of these crystals, due to the considerably blue-shifted absorption maximum (see Table 1 and Figure 5a), considerably lower NLO coefficients compared to the red-colored materials such as DAST are expected, based on the common nonlinearity-transparency trade-off (see Equation (11)). However, the measured NLO coefficients of BNA are surprisingly high, see Table 1, with the measured diagonal NLO coefficient of $d_{333}=234 \mathrm{pm} / \mathrm{V}$ at the fundamental wavelength of $1064 \mathrm{~nm}$ [56], which is however resonantly 
enhanced. From the measured dispersion of the refractive indices and using a two-level dispersion model according to Equation (10), one can estimate $d_{333} \approx 92 \mathrm{pm} / \mathrm{V}$ and $d_{333} \approx 72 \mathrm{pm} / \mathrm{V}$ for BNA at 1500-nm and 1900-nm fundamental wavelength, respectively. Considering the two-level dispersion model for both the NLO and the electro-optic coefficient, we can estimate the highest electro-optic coefficient of BNA is $r_{333}=24 \mathrm{pm} / \mathrm{V}$ at $1538 \mathrm{~nm}$ [100]. Although some experimental results suggest different values of the corresponding NLO and electro-optic coefficients $[58,59,100]$, BNA crystal has been very successfully used for THz-wave generation applications, in particular in the pump wavelength regime at around $800 \mathrm{~nm}$ that is less optimal for high-nonlinearity red materials [101-108].

Several research groups worldwide identified many other interesting groups of ionic and non-ionic crystals recently, but have not yet been extensively used for $\mathrm{THz}$ applications. Some more crystal types are mentioned in Section 4, where we discuss different organic THz sources. For recent reviews on the development of novel organic materials see $[15,30]$. On the other hand, also some older organic NLO crystals with a lower nonlinearity may be interesting for $\mathrm{THz}$ photonics, which was not considered at the time of their development more than two decades ago. For a comprehensive review of these crystals, see, e.g., Ref. [11]. Only a few of these crystals, e.g., COANP (2-cyclooctylamino-5-nitropyridine) [109], DANPH (4-dimethylaminobenzaldehyde-4-nitrophenylhydrazone), MNBA (4'-nitrobenzylidene-3acetamino-4-methoxyaniline), MTTNPH (5-(methylthio)-thiophenecarboxaldehyde-4-nitrophenyl hydrazone), PNP (2-(N-prolinol)-5-nitropyridine) have been investigated for their THz properties [110].

The natural morphology of the crystals is also very important for applications because the NLO response critically depends on the orientation of the interacting fields with respect to the crystal axes and therefore an appropriate sample orientation is essential. For crystals with a natural morphology that does not allow a direct use for applications, a very cumbersome and low-yield cutting procedure is most often needed. A big part of the success encountered by DAST, DSTMS and OH1 crystals is due to the very favorable natural morphology $[43,44,46]$ that allows for direct use in THz-applications, because of the plate-like morphology with the largest plane containing the polar axis, as illustrated in Figure 4. Another interesting case is the one of the HMQ-TMS crystal illustrated in Figure 4d, where a natural cleavage plane allows for relatively simple cleaving of samples with a desired orientation and thickness, which additionally results in high optical-quality parallel surfaces of the samples, without the need for additional polishing [47].

\section{THz-Wave Generation and Detection via NLO Processes}

In the early years of organic NLO crystals development, the most promising application considered was second-harmonic generation from the infrared radiation emitted e.g., from widely available infrared diode lasers to the visible radiation, motivated in particular by the very large NLO coefficients of organic materials, one order of magnitude larger compared to inorganic alternatives. Due to the huge progress in visible lasers development and the problems with photostability for second-harmonic generation, since the generated second-harmonic wavelengths are typically close to the absorption edge of these crystals, the interest has been moved to high-speed electro-optic applications $[6,25,26]$ and in particular to $\mathrm{THz}$ photonics applications discussed in this review, for which photostability is usually high enough even for commercial applications. In this section, we summarize the theoretical basis of THz-wave generation relevant for organic crystals, the difference-frequency generation and the optical rectification and then present some examples of organic $\mathrm{THz}$ sources in Section 4.

\subsection{Difference-Frequency Generation}

To induce a nonlinear polarization oscillating at a THz (angular) frequency $\omega_{\mathrm{THz}}$ based on difference-frequency generation, the interacting electric fields $\mathbf{E}\left(\omega_{1}\right)$ and $\mathbf{E}\left(\omega_{2}\right)$ should oscillate at closely spaced frequencies $\omega_{1}$ and $\omega_{2}$, so that their difference $\omega_{3}=\omega_{\mathrm{THz}}=\omega_{1}-\omega_{2}$ is in the $\mathrm{THz}$ 
frequency range. In order to efficiently generate $\omega_{\mathrm{THz}}$, the phase matching condition $\Delta \mathbf{k} \approx 0$ should be possibly well satisfied

$$
\Delta \mathbf{k}=\mathbf{k}_{\mathrm{THz}}-\left(\mathbf{k}_{1}-\mathbf{k}_{2}\right),
$$

where $\mathbf{k}_{\mathrm{THz}}$ is the wave vector of the THz-wave with the magnitude of $\left|\mathbf{k}_{\mathrm{THz}}\right|=2 \pi n_{\mathrm{THz}} / \lambda_{\mathrm{THz}}$ and $\mathbf{k}_{i}$ $(i=1,2)$ are the wave vectors of the interacting optical fields with the magnitude of $\left|\mathbf{k}_{i}\right|=2 \pi n_{i} / \lambda_{i}$. $n_{\mathrm{THz}}$ is the refractive index of the NLO material at the wavelength $\lambda_{\mathrm{THz}}$ of the generated THz field, while $n_{i}(i=1,2)$ are the refractive indices of the NLO material at the wavelengths $\lambda_{i}(i=1,2)$ of the two interacting fields $\mathbf{E}\left(\omega_{1}\right)$ and $\mathbf{E}\left(\omega_{2}\right)$, respectively. The phase matching condition is often quantified by the so-called coherence length $l_{c}$, which defines the maximum length of the NLO material, over which the generated field increases with the thickness

$$
l_{c}=\frac{\pi}{|\Delta \mathbf{k}|} .
$$

In the simplest case of the collinear difference-frequency generation, the three interacting waves propagate in the same direction and the corresponding wave vectors are all parallel. The resulting coherence length is then given by

$$
l_{c}=\frac{1}{2}\left(\frac{n_{\mathrm{THz}}}{\lambda_{\mathrm{THz}}}-\frac{n_{1}}{\lambda_{1}}+\frac{n_{2}}{\lambda_{2}}\right)^{-1}
$$

This expression can be additionally simplified for relatively small differences in frequencies of the two pump fields (up to several $\mathrm{THz}$ ) and in case the dispersion of the refractive index is relatively small, e.g., like in the infrared range for the organic crystals shown in Figure 5a. In this case, the dispersion in the optical range can be considered as $n_{2}=n_{1}+(\partial n / \partial \lambda)\left(\lambda_{2}-\lambda_{1}\right)$. This leads to the following coherence length for $\mathrm{THz}$-wave generation

$$
l_{c}=\frac{\lambda_{\mathrm{THz}}}{2\left(n_{\mathrm{THz}}-n_{g}\right)}
$$

where $n_{g}=n_{1}-(\partial n / \partial \lambda) \lambda_{1}$ is the group index of the optical wave.

For efficient generation of $\mathrm{THz}$ waves by difference-frequency generation, besides a high $\chi^{(2)}$ material susceptibility, the most important parameter is the low refractive index mismatch $\Delta n=n_{\mathrm{THz}}-n_{g}$ between the generated $\mathrm{THz}$ and the pump optical waves. Organic materials have an important advantage compared to standard inorganic NLO materials such as $\mathrm{LiNbO}_{3}$ because of the relatively weak dispersion of the refractive index between the optical and the $\mathrm{THz}$ frequency range, as discussed in Section 2.1. The phase-matching condition for THz-wave generation is in organics almost naturally satisfied, while for inorganic $\mathrm{NLO}$ crystals such as $\mathrm{LiNbO}_{3}$, more complex interacting-beam orientations are needed, e.g., tilted-pulse-front configuration [111]. The particular coherence lengths $l_{c}$ for organic NLO crystals are discussed in Section 3.4.

For difference-frequency generation in case of perfect phase-matching and neglecting the pump-light absorption, the visible-to-THz conversion efficiency is given by [20]

$$
\eta_{\mathrm{THz}}=\frac{\omega_{\mathrm{THz}}^{2} d_{\mathrm{THz}}^{2} L^{2} I_{0}}{2 \epsilon_{0} c^{3} n_{o}^{2} n_{\mathrm{THz}}} \exp \left(-\alpha_{\mathrm{THz}} L / 2\right)\left(\frac{\sinh \left(\alpha_{\mathrm{THz}} L / 4\right)}{\left(\alpha_{\mathrm{THz}} L / 4\right)}\right)^{2}
$$

where

$$
d_{\mathrm{THz}}=\frac{1}{4} n_{o}^{4} r
$$

is the NLO coefficient for THz-wave generation that scales with the electro-optic coefficient $r, L$ the length of the NLO material, $I_{0}$ the pump intensity, $\alpha_{\mathrm{THz}}$ the absorption coefficient at the $\mathrm{THz}$ frequency, $n_{o}$ and $n_{\mathrm{THz}}$ the refractive indices at the pump optical and the generated $\mathrm{THz}$ frequencies, 
respectively. Equation (16) shows that, besides phase-matching and minimal $\mathrm{THz}$ absorption $\alpha_{\mathrm{THz}}$ the main material figure of merit for $\mathrm{THz}$-wave generation $\mathrm{FM}_{\mathrm{THz}}$ can be defined as [6]

$$
\mathrm{FM}_{\mathrm{THz}}=\frac{d_{\mathrm{THz}}^{2}}{n_{o}^{2} n_{\mathrm{THz}}}=\frac{n_{o}^{6} r^{2}}{16 n_{\mathrm{THz}}} .
$$

Table 2 shows these parameters for several inorganic and organic NLO crystals at a wavelength that is as close as possible to phase matching. Conventional inorganic semiconducting crystals used for $\mathrm{THz}$-wave generation and detection such as ZnTe and GaP have much lower electro-optic coefficients compared to best inorganic electro-optic crystals such as $\mathrm{LiNbO}_{3}$, but are anyway widely used because of the possibility for phase matching that is not directly possible with $\mathrm{LiNbO}_{3}$ and other inorganic crystals with a high electro-optic coefficient. The organic crystals DAST, DSTMS, and OH1 show the largest figures of merit and they can be phase matched using pump lasers at telecommunication wavelengths in the range of $1300-1560 \mathrm{~nm}$. The particular phase matching properties at different pump wavelengths and the generated $\mathrm{THz}$ frequencies are discussed for these crystals in details in Section 3.4 and examples of $\mathrm{THz}$ sources based on difference-frequency generation in organic crystals are given in Section 4.1.

Table 2. Selection of organic and inorganic NLO materials that have been investigated for THz-wave generation and their most relevant parameters. Where possible, the parameters are given close to the optimal phase matching range: refractive index $n_{0}$ and the group index $n_{g}$ at the pump optical wavelength $\lambda$; refractive index $n_{\mathrm{THz}}$ in the THz range; the electro-optic coefficient $r$; the NLO coefficient $d_{\mathrm{THz}}$ for THz-wave generation according to Equation (17); the figure of merit $\mathrm{FM}_{\mathrm{THz}}$ for $\mathrm{THz}$-wave generation according to Equation (18).

\begin{tabular}{cccccccc}
\hline Material & $n_{\boldsymbol{o}}$ & $n_{\boldsymbol{g}}$ & $\boldsymbol{n}_{\mathrm{THz}}$ & $\boldsymbol{r}(\mathbf{p m} / \mathbf{V})$ & $\boldsymbol{d}_{\mathbf{T H z}}(\mathbf{p m} / \mathbf{V})$ & $\mathbf{F M}_{\mathbf{T H z}}(\mathbf{p m} / \mathbf{V})^{\mathbf{2}}$ & $\lambda(\mathbf{n m})$ \\
\hline $\mathrm{DAST}$ & 2.13 & 2.26 & $\sim 2.3$ & 47 & 240 & 5600 & 1500 \\
$\mathrm{DSTMS}$ & 2.08 & 2.19 & $\sim 2.2$ & 50 & 230 & 5800 & 1500 \\
$\mathrm{OH} 1$ & 2.16 & 2.33 & $\sim 2.3$ & 52 & 280 & 7500 & 1300 \\
$\mathrm{ZnTe}$ & 2.85 & 3.2 & $\sim 3.2$ & 4 & 66 & 170 & 800 \\
$\mathrm{GaP}$ & 3.12 & 3.36 & $\sim 3.35$ & 1 & 24 & 17 & 1000 \\
$\mathrm{LiNbO}_{3}$ & 2.16 & 2.22 & $\sim 5.0$ & 30 & 160 & 1100 & 1000 \\
\hline
\end{tabular}

\subsection{Optical Rectification}

While for difference-frequency generation two pump-optical beams at closely spaced narrow single frequencies are used, THz-wave generation by optical rectification employs a single pump pulse of a very short pulse length. An ultrashort laser pulse in the femtosecond range induces a quasi-static polarization in $\chi^{(2)}$-NLO materials through optical rectification, which acts as a source of a THz field. A short laser pulse has an intrinsically broad bandwidth, i.e., a transform-limited laser beam with a pulse length of $10 \mathrm{fs}$ or $200 \mathrm{fs}$ has a bandwidth of roughly $40 \mathrm{THz}$ or $2 \mathrm{THz}$, respectively. Different frequency components in such a pulse can mix with each other in an NLO material, producing a broadband $\mathrm{THz}$ pulse. In first approximation, considering non-depleted pump, negligible optical and $\mathrm{THz}$ absorption, plane-wave approximation and at perfect phase matching, the intensity of the generated $\mathrm{THz}$ wave is in frequency domain given by $[6,112]$

$$
\begin{aligned}
I\left(\omega_{\mathrm{THz}}, L\right) & =\frac{1}{2} \epsilon_{0} c n_{\mathrm{THz}}\left|E\left(\omega_{\mathrm{THz}}, L\right)\right|^{2} \\
& \approx \frac{d_{\mathrm{THz}}^{2} I_{0}^{2} \omega_{\mathrm{THz}}^{2} L^{2}}{2 \epsilon_{0} c^{3} n_{0}^{2} n_{\mathrm{THz}}}
\end{aligned}
$$


and the conversion efficiency by

$$
\eta=\frac{I\left(\omega_{\mathrm{THz}}, L\right)}{I_{0}}=\frac{d_{\mathrm{THz}}^{2} I_{0} \omega_{\mathrm{THz}}^{2} L^{2}}{2 \epsilon_{0} c^{3} n_{o}^{2} n_{\mathrm{THz}}}=\frac{1}{2 \epsilon_{0} c^{3}} \mathrm{FM}_{\mathrm{THz}} \omega_{\mathrm{THz}}^{2} I_{0} L^{2},
$$

where the same figure of merit $\mathrm{FM}_{\mathrm{THz}}$ as the one defined for difference-frequency generation in Equation (18) and listed in Table 2 is relevant. An analytical solution has been also derived for a more general case, considering both optical and THz-wave absorption and non-perfect phase matching, but still considering a non-depleted pump approximation [112,113]. In the plane-wave approximation and neglecting surface contributions, the generated electric field $E_{\mathrm{THz}}(\omega, \lambda, L)$ at the angular $\mathrm{THz}$ frequency $\omega_{\mathrm{THz}}=\omega$ pumped by an optical pulse with the central wavelength $\lambda$ is given by $[112,113]$

$$
E_{\mathrm{THz}}(\omega, \lambda, L)=\frac{2 d_{\mathrm{THz}} \omega^{2} I(\omega)}{\left(\frac{\omega}{c}\left(n_{\mathrm{THz}}+n_{g}\right)+i\left(\alpha_{\mathrm{THz}} / 2+\alpha\right)\right) n \epsilon_{0} c^{3}} \cdot \frac{\mathrm{e}^{i\left(\frac{\omega}{c} n_{\mathrm{THz}}+i \alpha_{\mathrm{THz}} / 2\right) L}-\mathrm{e}^{i\left(\frac{\omega}{c} n_{g}+i \alpha\right) L}}{\frac{\omega}{c}\left(n_{\mathrm{THz}}-n_{g}\right)+i\left(\alpha_{\mathrm{THz}} / 2-\alpha\right)},
$$

where $I(\omega)$ is the Fourier transform of the pump optical pulse $I(t)$ and $L$ the material thickness. Besides the NLO coefficient for THz-wave generation $d_{\mathrm{THz}}$, the following NLO material parameters are important: the refractive index $n_{\mathrm{THz}}$ and the absorption coefficient $\alpha_{\mathrm{THz}}$ at the THz angular frequency $\omega$, as well as the refractive index $n$, the optical group index $n_{g}$ and the absorption coefficient $\alpha$ at the optical wavelength $\lambda$. The second term in Equation (22) has a unit of length and its maximum is reached at exact phase matching (also called velocity matching) $n_{\mathrm{THz}}=n_{g}$ in case of negligible absorption. Its absolute value is referred to as the effective generation length $L_{\text {gen }}(\omega, \lambda, L)$ and is a useful parameter to analyze the thickness dependence of the generated $\mathrm{THz}$ spectrum, which strongly depends on the material dispersion [112]. A simpler figure of merit resulting from the expression (22), which however neglects the optical and $\mathrm{THz}$ absorption effects, is again the coherence length $l_{c}$, which is given by the same Equation (15) as for difference-frequency generation. The particular phase-matching properties at different pump wavelengths and the generated $\mathrm{THz}$ frequencies for organic NLO crystals are discussed in detail in Section 3.4 and examples of THz sources based on optical rectification in organic materials are given in Section 4.2.

The most common THz-wave generation scheme based on optical rectification employs a short fs pump pulse to produce a broadband $\mathrm{THz}$ pulse. Often, however, a single frequency $\mathrm{THz}$ source is desired, such as produced by difference-frequency generation for example. A tunable narrowband $\mathrm{THz}$ source can be also obtained by using a beating signal of two linearly chirped fs pulses with a variable delay as pump on a THz generator [114]. By controlling the length of the chirped pulse and the delay between the beating pulses, one can tune the frequency and the bandwidth of the generated narrowband $\mathrm{THz}$ pulses [114]. This method has been recently also demonstrated using organic $\mathrm{THz}$ sources $[78,82,115]$.

\section{3. $\mathrm{THz}$-Wave Detection}

The detection of the generated $\mathrm{THz}$ waves is often as challenging as $\mathrm{THz}$ generation. There are several possibilities used for $\mathrm{THz}$-wave detection, which also depend on the type of the $\mathrm{THz}$ source. We mention here the approaches that are most often used for organic crystalline $\mathrm{THz}$ sources. For difference-frequency generation systems that produce narrowband $\mathrm{THz}$ pulses, most often incoherent detectors such as Golay cells, bolometers and pyroelectric detectors are used. These detectors are commercially available and they can be directly used to determine the intensity of the generated $\mathrm{THz}$ radiation. For systems based on optical rectification, one can additionally use coherent detection methods, which are able to detect the amplitude and the phase of the oscillating $\mathrm{THz}$ field, i.e., the exact time dependence of the generated electric field $E_{\mathrm{THz}}(t)$. The coherent detection methods can give a lot of additional information and are therefore very attractive for applications. We briefly describe the most common methods that are used for coherent detection of $\mathrm{THz}$ fields 
produced by optical rectification in organic NLO crystals. Some of the often employed coherent detection methods for the optical rectification systems are illustrated in Figure 7.

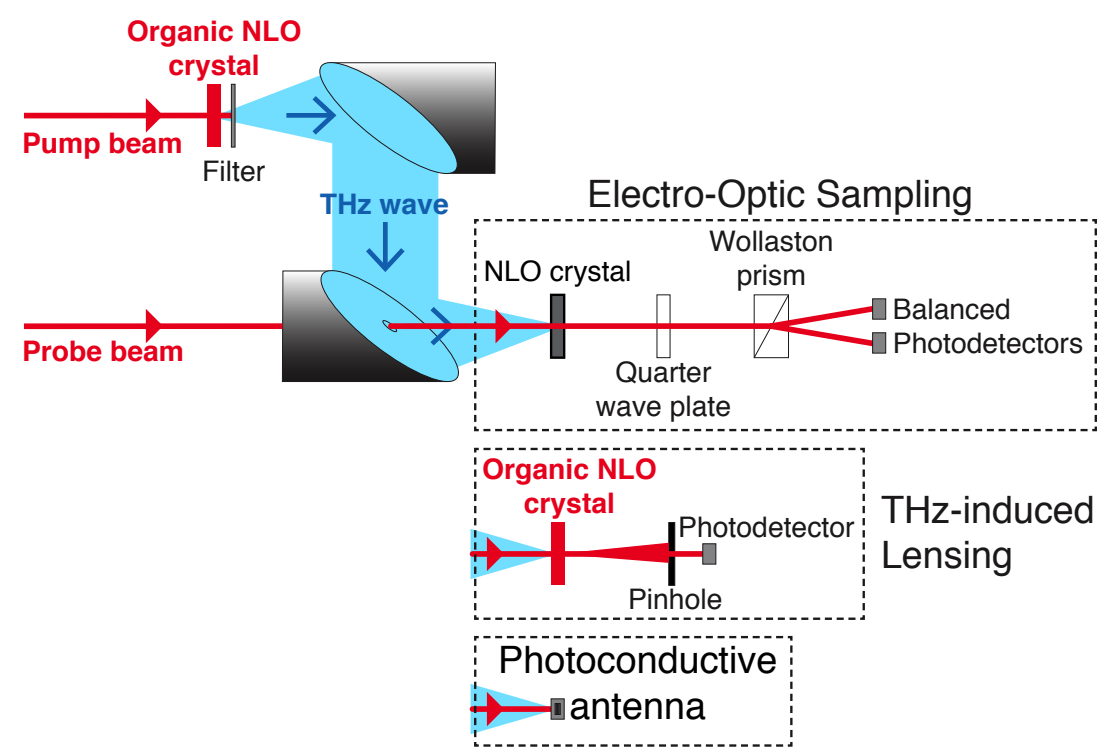

Figure 7. Schematics of a possible experimental geometry for THz-wave generation based on optical rectification with an organic electro-optic crystal as $\mathrm{THz}$ generator, indicating some of the possible coherent detection approaches: electro-optic sampling (EOS) with inorganic electro-optic crystals such as $\mathrm{ZnTe}$ or GaP, THz-induced lensing with organic electro-optic crystals, or detection with a photoconductive antenna. The pump and the probe fs beams can be of the same or of a different wavelength, but they have to be synchronized. The signal, proportional to the $\mathrm{THz}$ electric field $E_{\mathrm{THz}}(t)$, is measured as a function of the delay between pump and probe pulses.

Photoconductive antennas measure the transient photoconductivity induced by the probe optical field. When the generated THz electric field overlaps with the probe optical beam on a photoconductive antenna, a current is induced across the electrodes of the antenna and its magnitude and sign are proportional to the magnitude and the sign of the $\mathrm{THz}$ field. By delaying the probe pulse with respect to the $\mathrm{THz}$ pulse, a time-domain trace of the $\mathrm{THz}$ electric field $E_{\mathrm{THz}}(t)$ is obtained. Most commonly, the response of the photoconductive antennas is limited to a few $\mathrm{THz}$ only, but, using special antenna designs, broadband detection has also been demonstrated [116].

When fs optical pulses with relatively high pulse energies (in the order of $1 \mathrm{~mJ}$ ) are available, the detection is even possible in air by the so-called air-breakdown-coherent-detection (ABCD) [117,118].

Most commonly used technique for detecting the THz waves based on NLO crystals is the electro-optic sampling. This method employs electro-optic crystals with zero birefringence such as $\mathrm{ZnTe}$ or GaP [119]. When the THz field illuminates the detection electro-optic crystal, it induces a birefringence through the conventional electro-optic effect according to Equation (3). The induced birefringence changes the polarization state of the probe beam, which is usually detected using a combination of a quarter-wave plate, Wollaston prism, and two balanced photodiodes [119] (see Figure 7).

For highly birefringent materials such as organic NLO crystals, conventional electro-optic sampling detection cannot be used. Several alternative techniques have been proposed for birefringent materials [80,120-123]. A relatively simple approach is the so-called THz-induced lensing technique, which also relies on the electro-optic effect [121]. In this method, the refractive index changes induced by a focused $\mathrm{THz}$ electric field through the electro-optic effect are exploited. The spatially varying (focused) THz field induces a spatially varying refractive index change

$$
\Delta n(x, y, t)=-\frac{1}{2} n^{3} r E_{\mathrm{THz}}(x, y, t) .
$$


This time-varying refractive index profile acts as a time-varying optical lens and leads to focusing/defocusing of the probe beam, which can be detected by various common optical detection techniques $[121,124]$.

For the above mentioned electro-optic-based detection methods, the electro-optic sampling and the $\mathrm{THz}$-induced lensing, the same phase-matching considerations are valid as for $\mathrm{THz}$-wave generation with the corresponding material, considering the $\mathrm{THz}$ frequency limitations and the probe beam wavelength; i.e., for efficient detection, the $\mathrm{THz}$ refractive index $n_{\mathrm{THz}}$ and the optical group index $n_{g}$ for the detection pulse should match. In the same way as for detecting with the photoconductive antennas, by delaying the probe pulse with respect to the $\mathrm{THz}$ pulse, a time-domain trace of the $\mathrm{THz}$ electric field $E_{\mathrm{THz}}(t)$ is obtained by electro-optic sampling or THz-induced lensing (see Figure 7). Examples of the measured $\mathrm{THz}$ signals using different detection principles in systems based on optical rectification in organic crystals are presented in Section 4.2.

\subsection{Phase-Matching Characteristics for THz-Wave Generation in Organic NLO Crystals}

For efficient THz-wave generation by NLO processes (such as difference-frequency generation and optical rectification), it is essential to achieve phase matching (velocity matching) $n_{\mathrm{THz}} \simeq n_{g}$ between the fundamental optical and the generated THz waves, as discussed in Section 3. Because of the dispersion of the refractive indices in both ranges, the phase matching condition is strongly dependent on the wavelength $\lambda$ of the pump optical beam(s) and the frequencies $\omega$ of the generated $\mathrm{THz}$ beams. The dispersion of the optical group indices $n_{g}=n-(\partial n / \partial \lambda) \lambda$ is shown in Figure 8a next to the dispersion of the refractive indices $n(\omega)$ in the THz range (see Figure $8 b$ ) for selected organic NLO crystals.

For different materials, there are different ranges where the velocity matching condition $n_{\mathrm{THz}} \simeq n_{g}$ is reasonably well satisfied and is roughly marked by the shaded thick lines in Figure 8. DAST for example has a stronger phonon resonance near $1.1 \mathrm{THz}$ resulting in roughly two different regions: good velocity matching can be realized either for pump wavelengths between ca. $1000 \mathrm{~nm}$ and $1200 \mathrm{~nm}$, yielding $\mathrm{THz}$ waves between $0.2 \mathrm{THz}$ and $1 \mathrm{THz}$, or for pump waves with wavelengths between $1200 \mathrm{~nm}$ and $1700 \mathrm{~nm}$ for the generation of THz waves above $1.5 \mathrm{THz}$ [112,125]. For crystals with a blue shifted visible absorption edge such as HMQ-TMS, the best velocity matching ranges are also blue shifted, which is very important when selecting an organic $\mathrm{THz}$ generator that should be optimized for a certain pump laser source. The optical phonon resonances and molecular vibrations that occur in the THz frequency range (see Figure 3 ) induce some absorption of the generated $\mathrm{THz}$ radiation in organic NLO crystals (see the graphs for $\alpha_{\mathrm{THz}}(f)$ in Figure $8 \mathbf{b}$ ), which is also very important when optimizing these crystals for different desired frequency ranges. Note also that the properties in Figure 8 are only given along the polar axis of the selected NLO crystals. Several crystals also have notable off-diagonal $\chi^{(2)}$ components (see Table 1 ). Although these coefficients may be lower compared to the diagonal susceptibility coefficients (employed when both optical and THz beams are polarized along the polar axis), they may allow other velocity matching ranges. For DAST, for example, using the pump beams polarized along the crystalline $b$ axis (see Figure 4) generates $\mathrm{THz}$ radiation along the polar $a$ axis based on the off-diagonal $\chi_{122}^{(2)}$ susceptibility, which is velocity matched for the pump wavelengths close to $800 \mathrm{~nm}[75,112]$, an important wavelength of available high-power Ti:Sapphire fs laser sources.

Best conditions for velocity matching can be straightforwardly evaluated if the relevant optical parameters given in Figure 8 are known. The simplest figure-of-merit for the velocity matching is the coherence length $l_{c}(\lambda, f)$ given by Equation (15) and is shown in Figure 9 for selected organic materials obtained using the parameters in Figure 8 as a function of the pump optical wavelength $\lambda$ and the generated THz frequency $f=c / \lambda_{\mathrm{THz}}$. 
(a)
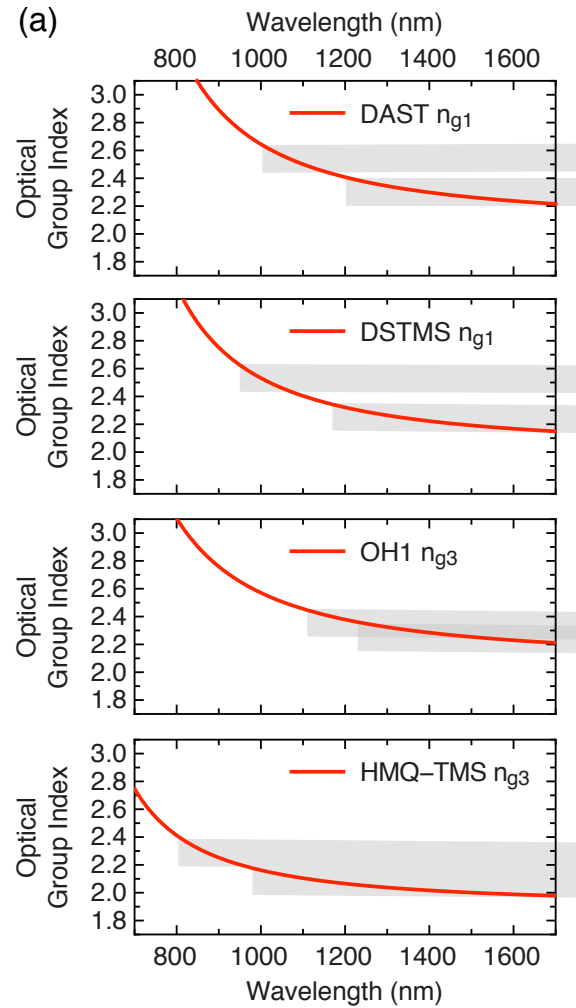

(b)
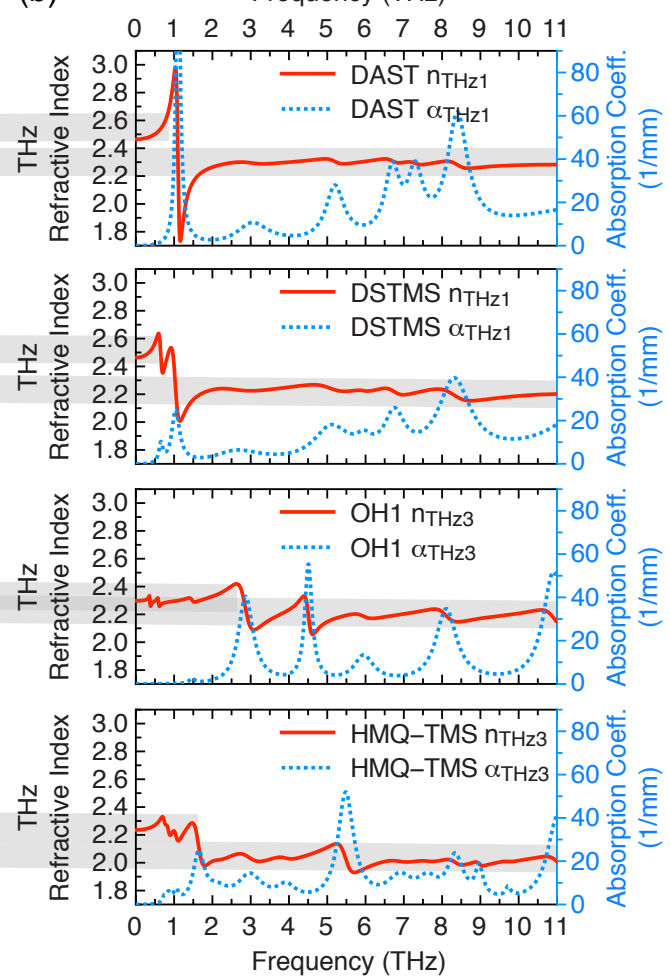

Figure 8. (a) optical group index $n_{g}(\lambda)$ as a function of wavelength for selected organic crystals; (b) optical properties in the $\mathrm{THz}$ range: the refractive index $n_{\mathrm{THz}}(f)$ (red solid curves) and the absorption coefficient $\alpha_{\mathrm{THz}}(f)$ (blue dotted curves, right scale) as a function of frequency $f=\omega /(2 \pi)$. The corresponding properties are given for selected organic crystals along their polar axis, which is most often employed in THz applications. The $n_{g}(\lambda)$ are obtained from the dispersions $n(\lambda)$ shown in Figure 5a. The THz properties $n_{\mathrm{THz}}(f)$ and $\alpha_{\mathrm{THz}}(f)$ are according to the following references: DAST [126-128], DSTMS [129], OH1 [91], HMQ-TMS [53,83].

For highly nonlinear organic NLO crystals DAST and DSTMS presented in Figure 9, the best pump wavelength range is at around telecommunication wavelengths, which is very interesting given the availability of very compact and relatively low-cost fs fiber lasers [125]. The generation of THz waves with frequencies at around $1.1 \mathrm{THz}$ is in DAST limited due to a strong optical phonon vibration [130]. In DSTMS (DAST derivative with an isomorphic structure), the optical phonon absorption near $1 \mathrm{THz}$ is suppressed (see Figure $8 b$ ), most probably due to a heavier counter anion, and therefore this material is superior to DAST within this frequency range [72]. In a broader THz frequency range up to about $10 \mathrm{THz}$ (for which the $\mathrm{THz}$ refractive index data is available), DSTMS shows similar $\mathrm{THz}$ velocity-matching properties compared to DAST.

$\mathrm{OH} 1$ crystal is a non-ionic crystal and has even higher figure of merit for THz-wave generation (see Table 2) compared to the stilbazolium salts DAST and DSTMS. OH1 has optimum velocity-matching between $1200 \mathrm{~nm}$ and $1460 \mathrm{~nm}$ for $0.3-2.5 \mathrm{THz}$, and has no absorption at about $1 \mathrm{THz}$ but at about $3 \mathrm{THz}$ [85]. Above $3 \mathrm{THz}$, the optimal phase matching conditions for $\mathrm{OH} 1$ are shifted to longer wavelengths, which is less optimal considering the available pump laser sources. Laser sources at around $1550 \mathrm{~nm}$ are not best suitable for $\mathrm{OH} 1$ due to a smaller absorption band in the optical range at this wavelength (see Figure $5 b$ ). In any case, $\mathrm{OH} 1$ has the highest efficiency among presently known organic crystals, at least in the lower $\mathrm{THz}$ frequency range below $3 \mathrm{THz}$ [85]. 

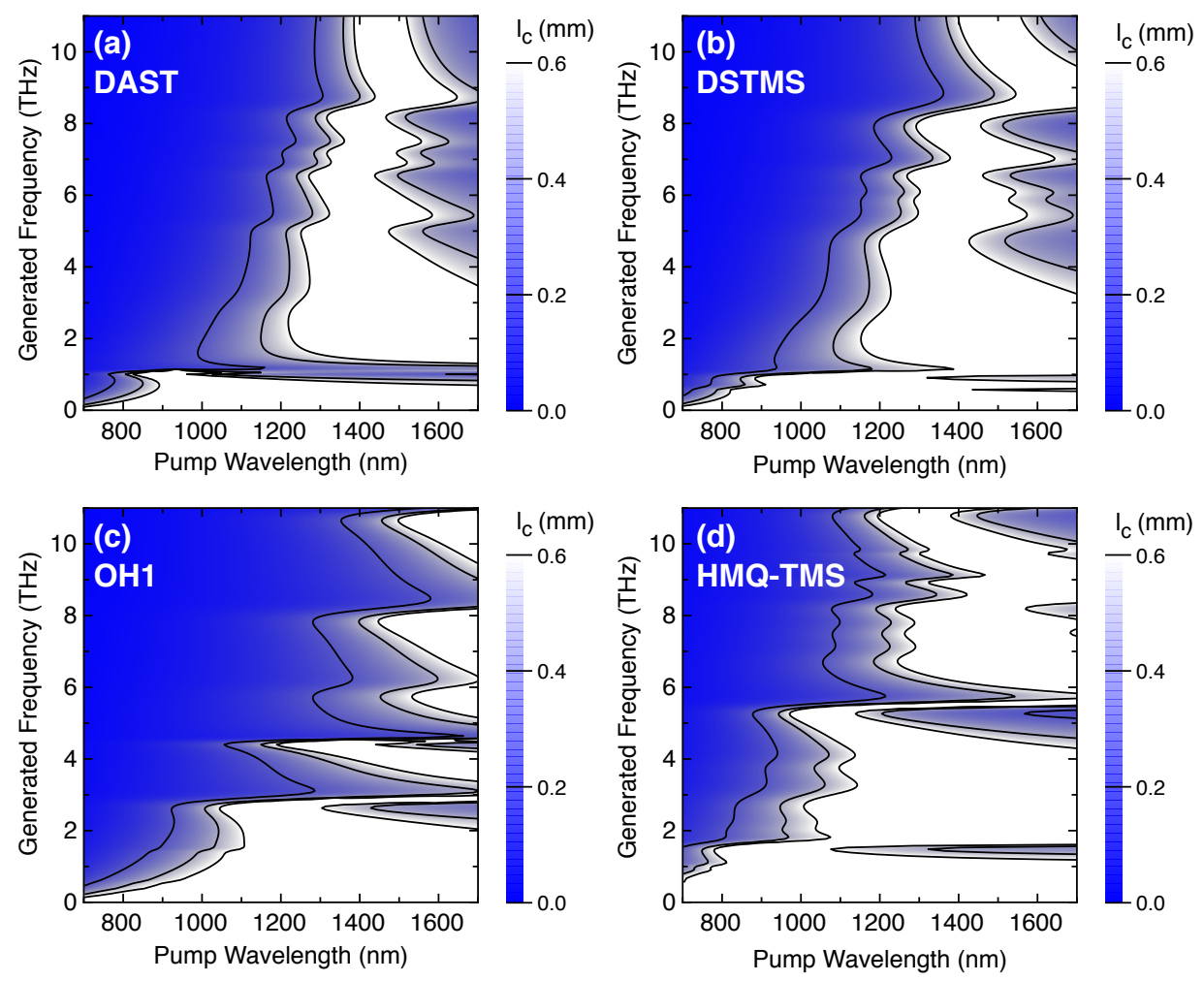

Figure 9. Coherence length $l_{c}$ for THz-wave generation in (a) DAST; (b) DSTMS; (c) OH1; and (d) HMQ-TMS as a function of the pump optical wavelength $\lambda$ and the generated THz frequency $f$. The white area represents coherence lengths larger than $0.6 \mathrm{~mm}$ and therefore best velocity matching.

For the quinolinium salt HMQ-TMS, see Figure 9d, the best phase-matching range is shifted towards shorter wavelengths compared to DAST and DSTMS, which is an advantage for using certain widely pump-wavelength sources at around $1000 \mathrm{~nm}[53,83,84]$.

Note that the measurements of material parameters required for evaluating $l_{c}$ may be very time consuming and often also have a limited precision, therefore these figures only give a first approximation for the coherence length and are only available for a few organic NLO crystals. Some other organic NLO crystals, for which phase matching has been studied in more detail, are OHQ-T [131], BNA [103] and COANP [109]. BNA and COANP are, for example, interesting, although having somewhat lower nonlinearity, because their absorption is considerably blue shifted (see Figure 5a for BNA) and therefore they allow for velocity matching at around the Ti:Sapphire laser wavelength of $800 \mathrm{~nm}[103,107,109]$.

The velocity-matching evaluation based on the coherence length $l_{c}$ as presented in Figure 9 does not take into account the influence of the optical and the THz-wave absorption. Based on the analytical solution for the THz electric field given by Equation (22), an equivalent parameter $l_{\text {opt }}$ has been defined, the so-called optimum crystal length $l_{\text {opt }}$ [112], which considers both optical/THz absorption and optical/THz group/refractive indices. The corresponding plots of $l_{\text {opt }}$ as a function of $f$ and $\lambda$ for DAST, DSTMS, OH1 and HMQ-TMS can be found in Ref. [15]. The main conclusions about the best velocity-matching ranges remain, however, very similar to those resulting from Figure 9.

It is usually assumed that the NLO coefficient for THz-wave generation $d_{\mathrm{THz}}$, which is important for the THz-wave generation efficiency beside phase matching (see Equations (16) and (22)), is approximately constant in the $\mathrm{THz}$ frequency range. This may not be the case due to the phonon and vibrational modes in this range and the NLO susceptibility in this range can be also considerably frequency dependent similarly to the linear optical properties shown in Figure 8b. A recent experiment shows that $d_{\mathrm{THz}}$ may be even strongly enhanced at certain $\mathrm{THz}$ frequencies [132], which then additionally affects the final features in the generated $\mathrm{THz}$ spectrum in addition to phase matching. 
There is a strong need to increase the variety of different NLO crystals that would allow for phase matching for various combinations of available pump optical and the desired generated $\mathrm{THz}$ frequency ranges. This is one of the reasons that the development of novel organic NLO crystals is being continued $[15,30]$.

\section{Organic Crystalline THz Sources}

Over the last two decades, the interest in organic THz sources has been steadily increasing due to their high THz-wave generation efficiency and the ability to achieve ultra-high bandwidths as well as high THz electric fields. Zhang et al. [133] were the first to demonstrate the generation of THz-waves by optical rectification in organic DAST crystal. His group obtained results of one and two orders of magnitude larger than that from $\mathrm{GaAs}$ and $\mathrm{LiTaO}_{3}$ inorganic crystals, respectively. This work triggered other researchers around the globe and in the following years, there were many reports of new achievements with organic THz sources. In this section, we briefly describe some of the most relevant research results, mostly from the past decade in the area of ultra-high bandwidth and high electric field THz-wave generation with organic crystals. Some of them involve new recently developed materials, while others employ well known organic crystals used in new studies.

\subsection{THZ Sources Based on Difference-Frequency Generation}

By using the process of difference-frequency generation, narrowband and in most cases frequency-tunable $\mathrm{THz}$ wave generation in various NLO crystals has been demonstrated. While the accessible $\mathrm{THz}$ wavelength range is intrinsically limited by the absorption of the NLO material employed, it is difficult to generate waves above $5 \mathrm{THz}$ efficiently in inorganic crystals due to strong optical phonon resonances. The $\mathrm{THz}$ sources based on difference-frequency generation in organic NLO crystals are characterized by a wide tunability range, often beyond $20 \mathrm{THz}$ (see Figure 10), a narrow single frequency output with linewidths that may be below $5 \mathrm{GHz}$ [134] and high $\mathrm{THz}$ pulse energies beyond $500 \mathrm{~nJ}$ [97]. The typical dynamic range of such systems is about $30 \mathrm{~dB}$ (see Figure 10), limited by incoherent $\mathrm{THz}$ detection techniques employed (see Section 3.3).

The most often used dual-wavelength source is a nanosecond (in some cases picosecond) optical parametric oscillator that generates two closely spaced pump wavelengths that are possibly in the phase-matching range of a particular NLO crystal employed. In many of the reported investigations, DAST has been used as a THz-wave generation crystal [106,134-151], while more recently other organic NLO crystals such as BNA[103,106], DSTMS [73] and OH1 [89,91,95,97,152] have been employed, with different benefits and differences in particular with respect to the achievable efficiencies, $\mathrm{THz}$ frequency ranges and optimal pump wavelengths of the dual-wavelength source.

A slightly non-collinear difference-frequency generation geometry may be used to achieve exact phase-matching conditions and improve the efficiency in a desired THz range [144]. Specially cut crystals may be used to optimize the phase matching at other pump wavelengths $[147,148]$. The possibility of quasi-phase matching has also been demonstrated in case the available dual-wavelength source is out of the best phase-matching conditions of a particular crystal [91]. Intrinsic gaps in the achievable spectrum are observed due to optical phonon and molecular vibrations (see Figure $8 b$ ), as shown in Figure 10a,b [106]. An interesting possibility to reduce these gaps is to combine the output generated by difference-frequency generation in different organic NLO crystals to obtain a more homogeneous $\mathrm{THz}$ spectrum, as demonstrated for DAST and BNA (see Figure 10c [106]).

In general, it is relatively straightforward to produce narrowband $\mathrm{THz}$ waves by differencefrequency generation in organic NLO crystals, which may be most often phase matched without the need for a special crystal cut at certain pump-wavelength ranges [135-146]. It is more challenging to develop an appropriate pump-beam pair with a desired separation in the $\mathrm{THz}$ frequency range. These systems are presently mostly based on high-power optical parametric oscillators and therefore relatively bulky and sensitive. More compact generation systems can be achieved by using optical rectification discussed in the following section. 

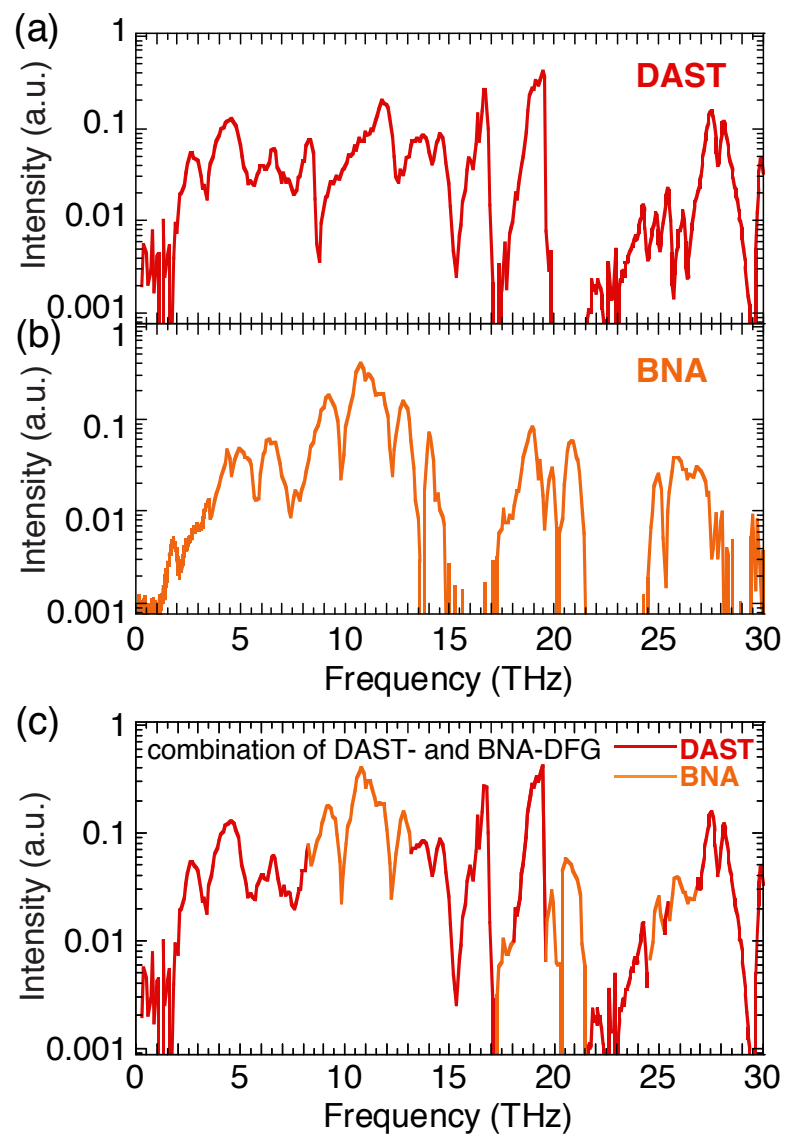

Figure 10. An example of THz-wave generation by difference-frequency generation in (a) DAST (0.3 mm thick) and (b) BNA (0.3 mm thick) using a dual-wavelength optical parametric oscillator emitting in the wavelength range 1200-1600 nm for DAST and 800-1300 nm for BNA; (c) an improved broadband spectrum achieved by using a different crystal at different $\mathrm{THz}$ frequencies. Reprinted from Ref. [106] with permission from The Optical Society (OSA).

\section{2. $\mathrm{THz}$ Sources Based on Optical Rectification}

The $\mathrm{THz}$ sources based on optical rectification in organic crystals are presently more widely employed compared to the sources based on difference-frequency generation. The $\mathrm{THz}$ electric field strength and its spectrum achieved by these sources depend on the characteristic properties of a particular THz generator, its nonlinearity and phase matching (see Section 3.4), as well as on the characteristics of the particular pump laser source through the source term $I(\omega)$ in Equation (22). The shorter the pump pulses, the broader the $\mathrm{THz}$ spectrum that can be achieved by optical rectification. For example, using 17-fs pump pulses, it was possible to generate a spectrum extending from 0.1 to beyond $25 \mathrm{THz}$ in DAST crystal by optical rectification [153], and using 5-fs short pulses, the spectrum extended even up to $200 \mathrm{THz}$ [154].

THz-wave generation by optical rectification in organic crystals has been first demonstrated using DAST in 1992 [133], followed by investigations in many different groups, initially only in DAST (see, e.g., Refs. [93,112,120,130,143,147,153-159]), and later extended to other organic crystals, such as DSTMS (see, e.g., Refs. [72,76,79,81,93,94,160,161]), OH1 (see, e.g., Refs. [85,93,94,96-98]), HMQ-TMS (see, e.g., Refs. $[53,83,84]$ ), BNA $[103,107]$ and many others (see some references from the last year only [132,162-167]). The variety of publications on different materials used for THz-wave generation is also because optical rectification has been recently often used as a useful technique to evaluate the potential of newly developed NLO organic crystals for THz photonics.

The final system specifications for applications such as e.g., needed for $\mathrm{THz}$ spectroscopy also strongly depend on the employed detection technique. A simplified example of a possible experimental 
set-up based on optical rectification is illustrated in Figure 7. The incoming pump laser pulse is split into a pump beam and a probe beam. The pump beam is used to generate a THz pulse in an organic NLO crystal and is then blocked by a filter transparent to $\mathrm{THz}$ radiation. For the optics to guide $\mathrm{THz}$ beams, parabolic or ellipsoidal mirrors are mostly used and different mirror combinations can be used to optimize the system for different $\mathrm{THz}$ photonics applications. The $\mathrm{THz}$ beam can be measured directly using incoherent $\mathrm{THz}$ detectors or coherently by overlapping $\mathrm{THz}$ pulses and probe optical pulses in a detector material, for which several different approaches are commonly used (see Section 3.3). The coherent detection, i.e., the measurement of the $\mathrm{THz}$ electric field as a function of time $E_{\mathrm{THz}}(t)$, is most often achieved by varying the relative path length of pump with respect to probe pulses with a computer-controlled optical delay line. In some cases, e.g., when different materials are used for generation and detection with different optimal operation wavelength, probe pulses with a different wavelength can be used, which can be achieved e.g., by using a second-harmonic of the pump beam or a direct output of a Ti:Sapphire laser in case an additional optical parametric amplifier is used for the pump beam.

For table-top THz sources, particularly for those aiming at high-power $\mathrm{THz}$ pulses that can be used for nonlinear THz photonics (see Section 5.1), conventional femtosecond Ti:Sapphire lasers, combined with high-power optical parametric amplifier systems, are most often employed. Conventional Ti:Sapphire lasers emit at the wavelength of around $800 \mathrm{~nm}$ and they can be directly used only for organic crystals that allow for phase matching at this wavelength, such as BNA [107,108], COANP [109] and some other recently developed organic crystals, e.g., crystals based on the 2-(4-hydroxystyryl)-1-methylquinolinium (OHQ) chromophore [168] or the 4-(4-hydroxystyryl)-1methyl-pyridinium (OHP) chromophore [167]. For highly NLO crystals such as DAST, DSTMS (see Figure 9), wavelengths of above $1200 \mathrm{~nm}$ are optimal, which is why an additional high-power optical parametric amplifier seeded by a Ti:Sapphire laser is used for these sources to convert the output to longer wavelengths. With such a Ti:Sapphire-based pump system, THz electric fields exceeding $150 \mathrm{MV} / \mathrm{m}$ [93] or even beyond $8 \mathrm{GV} / \mathrm{m}$ using special focusing optimization techniques have been demonstrated [94]. Figure 11a shows time domain traces achieved with such a system for DSTMS and $\mathrm{OH} 1$, where the pump wavelength has been chosen as the optimum for each of the crystals, $1500 \mathrm{~nm}$ and $1350 \mathrm{~nm}$, respectively. The spectral shape (see Figure 11b) such as the gaps in the spectrum for $\mathrm{OH} 1$, are due to the phonon and vibrational modes in this crystal, which corresponds well to the measured $\mathrm{THz}$ properties shown in Figure $8 \mathrm{~b}$ and the theoretical calculations of phonon modes in OH1 [169].

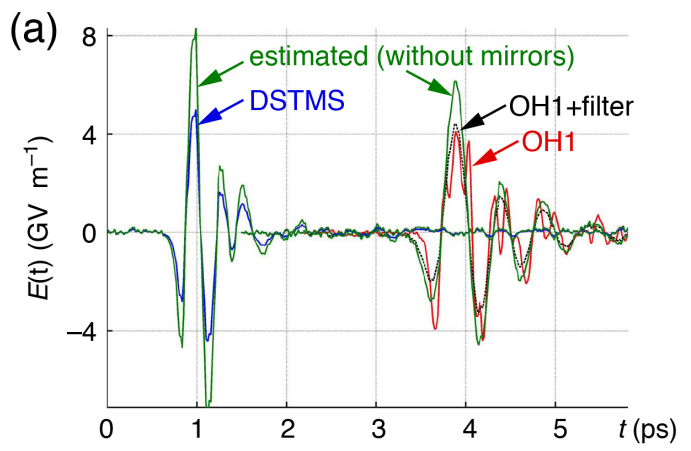

(b)

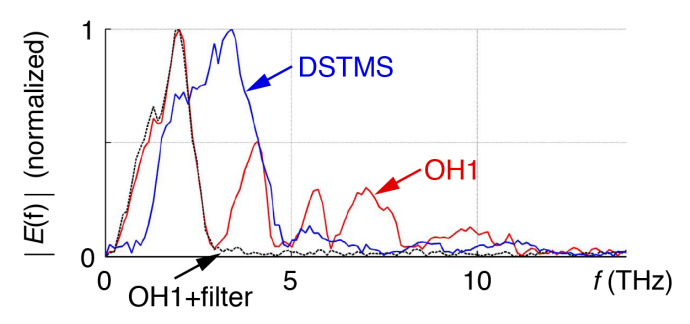

Figure 11. Intense THz-wave generation using a high-power Ti:Sapphire/optical parametric amplifier system (65 fs pulse duration, $100 \mathrm{~Hz}$ repetition rate) in DSTMS (0.44-mm thick, $1500 \mathrm{~nm}$ pump wavelength and $3.8 \mathrm{~mJ}$ pump energy, blue curves) and in $\mathrm{OH} 1$ (0.48-mm thick, $1350 \mathrm{~nm}$ pump wavelength and $3.5 \mathrm{~mJ}$ pump energy, red curves without and black dotted curves with a $3-\mathrm{THz}$ low-pass filter): (a) THz time-domain signals detected by air-breakdown-coherent-detection and (b) the corresponding amplitude spectra. The green curves in (a) represent the estimated generated electric field $E_{\mathrm{THz}}(t)$ considering the mirror losses, with a maximum of $8.3 \mathrm{GV} / \mathrm{m}$ for DSTMS and $6.2 \mathrm{GV} / \mathrm{m}$ for $\mathrm{OH} 1$ [94]. Reprinted from Ref. [94] with permission from Springer Nature. 
One interesting alternative to Ti-Sapphire-based pump systems is a high-energy Cr:Forsterite laser emitting at $1250 \mathrm{~nm}$, which is still in the optimal pump range for the state-of-the-art organic NLO crystals (see Figure 9). With Cr:Forsterite femtosecond pump, THz electric fields higher than $1 \mathrm{GV} / \mathrm{m}$ in organic crystals DAST, DSTMS and OH1 have been generated [74,170,171].

With the variety of organic NLO crystals that are being developed, the possibility for phase matching should be also soon available for many other available high-power pump fs laser systems. For example, an ytterbium laser operating at $1030 \mathrm{~nm}$ [84] has been successfully used in combination with HMQ-TMS crystal, for which the best pump wavelength range is blue-shifted compared to DAST, DSTMS and OH1 (see Figure 9), also yielding high THz electric fields with a peak of about $20 \mathrm{MV} / \mathrm{m}$ [84].

In addition to high-power systems, relatively compact and ultra-broadband THz systems based on optical rectification in organic NLO crystals can be realized based on compact and relatively low-cost femtosecond laser sources operating at around $1560 \mathrm{~nm}$, which is about the best phase-matching range for the state-of-the-art organic crystals such as DAST and DSTMS. Since the available pump pulse energies of these fs lasers are relatively small (in the nJ-range, which is six orders of magnitude smaller in comparison with the values up to several $\mathrm{mJ}$ for the high-power systems, see, e.g., the values in the caption of Figure 11), these systems do not provide an intense $\mathrm{THz}$ power, but they are still very interesting in combination with a sensitive detection technique, providing a high dynamic range needed e.g., for THz time-domain spectroscopy applications. Two of such examples based on a DSTMS organic crystal as $\mathrm{THz}$ generator are presented in Figures 12 and 13. In the first example, a highly sensitive photoconductive antenna optimized at $1560 \mathrm{~nm}$ wavelength has been used and in the second example, another DSTMS crystal has been used for the THz-induced lensing detection. Using organic crystals as both $\mathrm{THz}$ generation and detection materials enables achieving an ultra-broad $\mathrm{THz}$ spectral bandwidth beyond $15 \mathrm{THz}$, profiting from the phase-matching on both generation and detection part.

(a)

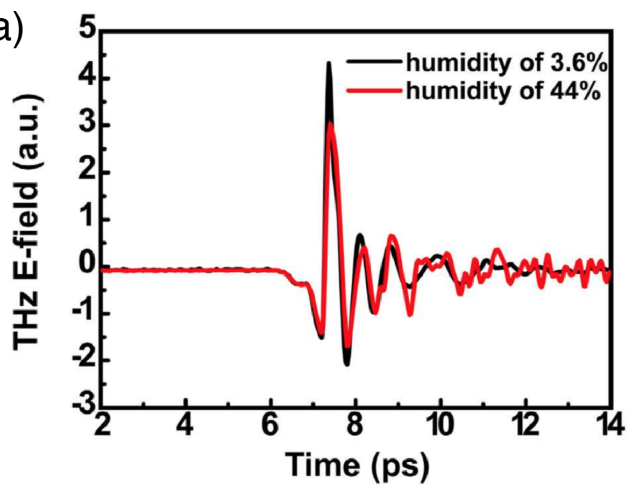

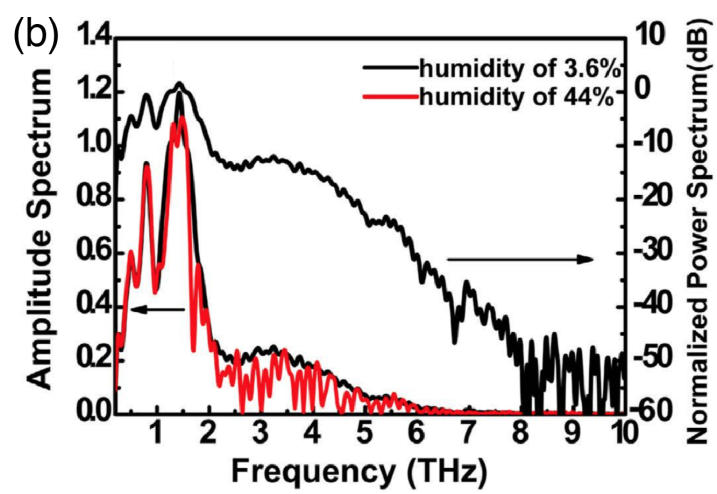

Figure 12. (a) THz time-domain signal and (b) the corresponding amplitude and power spectrum achieved in a compact terahertz setup based on DSTMS (0.3 mm thick) as THz generator and photoconductive antenna as $\mathrm{THz}$ detector and a pump laser with pulse length of $<100 \mathrm{fs},>200 \mathrm{~mW}$ average power, $80 \mathrm{MHz}$ repetition rate and $1560 \mathrm{~nm}$ central wavelength [79]. The red lines represent signals measured in air with a humidity of $44 \%$ and black lines are those obtained in dry-air environment. Reprinted from Ref. [79]. Licensed by Creative Commons CC BY 4.0.

One drawback of broadband systems based on optical rectification in organic NLO crystals is the modulation of the resulting $\mathrm{THz}$ spectra (see, e.g., the spectrum in Figure $11 \mathrm{~b}$ for $\mathrm{OH} 1$ and in Figure $13 \mathrm{~b}$ for DSTMS). This is due to the intrinsic phonon and vibrational modes of the generating materials, resulting in $\mathrm{THz}$ absorption peaks as shown in Figure 8b, leading to the absorption of the generated $\mathrm{THz}$ waves and varying phase-matching figures. To avoid gaps in the spectrum, a solution based on exchanging between different crystals at different $\mathrm{THz}$ frequencies as proposed for the systems based on difference-frequency generation (see Figure 10c) is here not applicable because the whole spectrum is generated and detected simultaneously through the time-domain traces $E_{\mathrm{THz}}(t)$. 
Therefore, one important challenge for future development of novel organic NLO crystals for $\mathrm{THz}$ photonics is to reduce the strength of these vibrations. This can be done through molecular engineering, but this is unfortunately still extremely difficult to control because even a slight chemical change of the constituting molecules can considerably change the crystalline packing and the crystal growth ability [172]. Another recently proposed approach combines two complementary organic NLO crystals in a combined structure as a tandem THz generator [54]. Using a tandem consisting of HMQ-TMS and $\mathrm{OH} 1$, it was shown that a certain compensation of phonon-absorption gaps in the generated $\mathrm{THz}$ spectra is possible for optimized crystal combinations [54].
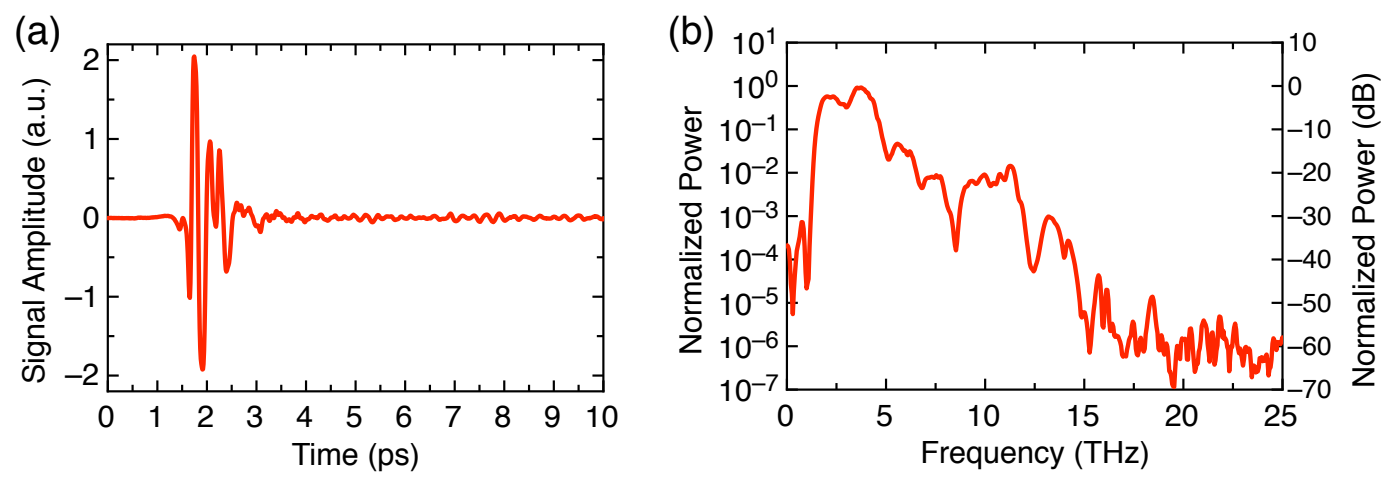

Figure 13. (a) THz time-domain signal and (b) the corresponding power spectrum achieved in dry-air environment using a compact terahertz setup based on a DSTMS crystal as THz generator and another DSTMS crystal as THz detector (each crystal was about $0.6 \mathrm{~mm}$ thick) and a pump laser with pulse length of $40 \mathrm{fs}, 190 \mathrm{~mW}$ average power, $100 \mathrm{MHz}$ repetition rate and $1560 \mathrm{~nm}$ central wavelength [173].

\section{Applications of Organic THz Sources}

During the last decade, $\mathrm{THz}$ systems based on organic single crystalline $\mathrm{THz}$ sources reached many areas of applications from fundamental science to real-world applications. The research and practical applications such as $\mathrm{THz}$ spectroscopy and $\mathrm{THz}$ imaging in laboratories worldwide performed by organic NLO crystals are still more exceptional in comparison with competitive $\mathrm{THz}$ systems based on photoconductive antennas or inorganic NLO crystals that are more common, also because photoconductive antennas and inorganic NLO crystals are much more widely available. Nevertheless, the use of organic crystalline $\mathrm{THz}$ sources in fundamental science and development is moving to other interdisciplinary fields [174-179], which indicates a great potential for the future transfer of organic-crystal based $\mathrm{THz}$ spectroscopic and imaging systems into industrial applications. According to the THz science and technology roadmap prepared by Dhillon et al. [1], one can expect that organic $\mathrm{THz}$ sources will enter other fields of applications similarly as $\mathrm{THz}$ systems based on photoconductive antennas or inorganic NLO crystals. Furthermore, the last experiments showed that organic NLO crystals in a combination with photoconductive antennas provide good performance suitable for various applications [79]. Due to the possibility to reach very high $\mathrm{THz}$ electric fields with organic NLO crystals, several demonstrations of fundamental THz nonlinear photonics have been possible (see Section 5.1) using these sources, especially interesting due to the possibility to control matter with $\mathrm{THz}$ beams. The following sections summarize the past and current directions of use as well as provide an opinion on the future challenges and opportunities of organic crystalline $\mathrm{THz}$ sources.

\subsection{THz Nonlinear Photonics}

Recent progress in generation of intense $\mathrm{THz}$ pulses opened a range of new possibilities to study nonlinear physical phenomena. Interaction between mater and electric field produces various linear and nonlinear effects that can modify material properties. When a high external field is applied, the induced polarization inside the medium is a sum of the linear and nonlinear components (similarly as in the optical range described by Equation (2)), giving rise to many nonlinear effects $[180,181]$, such as 
coherent $\mathrm{THz}$ manipulation of quantum states, high-order harmonic generation, nonlinear optical processes, and nonlinear transport phenomena in solids [182]. Various $\mathrm{THz}$ generation techniques are employed to achieve intense $\mathrm{THz}$ fields needed for nonlinear effects and most of them are based on optical rectification where either $\mathrm{LiNbO}_{3}$ [182-186] or organic NLO crystals are used. Here we summarize some of the reported examples of nonlinear $\mathrm{THz}$ research based on organic crystalline THz sources.

DSTMS THz emitter crystal was used for generating THz pulses with a high peak electric field of about $300 \mathrm{kV} / \mathrm{cm}$ for a system enabling the THz Kerr effect spectroscopy and two-dimensional terahertz-terahertz-Raman spectroscopy $[77,187,188]$, demonstrating a 2D experimental technique for measuring the nonlinear response of liquids in the $\mathrm{THz}$ field. The terahertz-terahertz-Raman pulse sequences that are sensitive to intermolecular modes (e.g., molecular orientational alignment) were employed to initiate intramolecular vibrational modes of liquids (see Figure 14). Vibrational coherence arising from intramolecular modes were observed in liquids such as diiodomethane at $3.66 \mathrm{THz}$ and in simple halogenated liquid carbon tetrachloride at $6.50 \mathrm{THz}$ [187]. These experiments open the door to $\mathrm{THz}$ spectroscopy that can study the dynamics of condensed-phase molecular systems such as the ability of liquids to solvate different compounds, the dynamics of biological macromolecules such as polypeptides, protein folding and DNA internal conversion as well as for amorphous solids and hydrogen-bonded liquids.

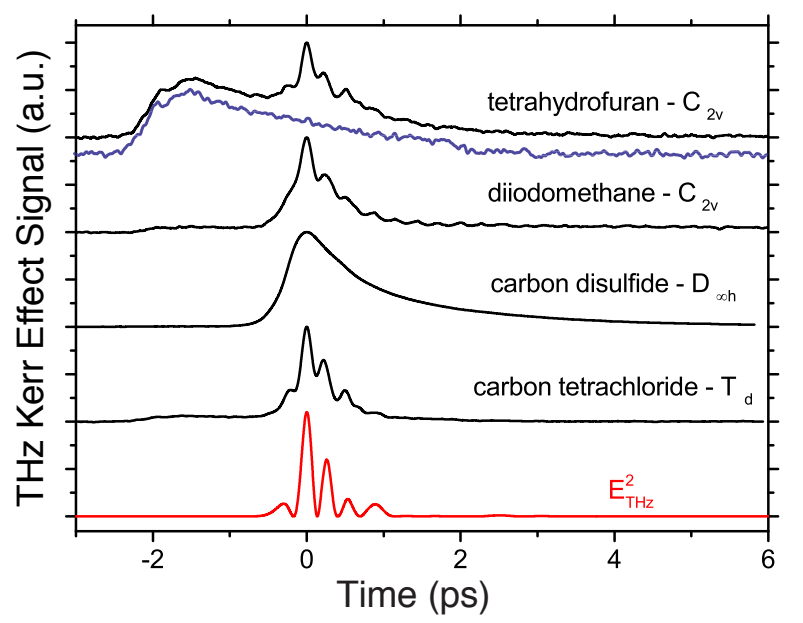

Figure 14. Measured $\mathrm{THz}$ Kerr-effect signals, generated by a terahertz-terahertz-Raman pulse sequence, of various liquids (black curves) [187]. Vibrational coherences are visible as oscillations in the measured signal. The bottom red trace is the square of the applied THz electric field and the blue trace below the tetrahydrofuran data shows the empty cuvette background response. Reprinted from Ref. [187] with permission from AIP Publishing.

Instantaneous Kerr nonlinearity and the retarded alignment of air molecules $\mathrm{CO}_{2}, \mathrm{~N}_{2}$, and $\mathrm{O}_{2}$ when triggered by intense $\mathrm{THz}$ pulses have been demonstrated by Shalaby et al [189], for which intense single-cycle THz pulses generated in a DSTMS crystal have been employed [94]. The same group demonstrated the visualization of broadband $\mathrm{THz}$ radiation in the $5-13 \mathrm{THz}$ frequency range, based on nonlinear $\mathrm{THz}$ absorption mechanism in the standard silicon charge-coupled (CCD) sensor [190]. Intense $\mathrm{THz}$ pulses have been shown to induce heat-induced demagnetization in the ferromagnetic nickel [191] and to change dielectric properties and simultaneous electronic and magnetic response in a cobalt thin film [192]. Chefonov et al. investigated n-doped silicon with high-field $\mathrm{THz}$ transmission experiment where they observed huge transmittance enhancement of approximately 90 times under high THz field strengths generated by a DSTMS crystal [161]. The observable high increase in the transmission of $\mathrm{n}$-doped silicon in the $\mathrm{THz}$ range by almost two orders can lead to the development of novel THz absorbers and THz field controlled electronic devices [161]. An ultrafast THz-induced optical modulator for pulse switching in a thin diamond window has also been demonstrated [193]. 
Vicario et al. reported on extreme laser spectral broadening and a change in optical transmission in GaP induced by a strong THz field [194]. DSTMS crystals were used in the experiment with thin aluminum and nickel films, where damage produced by a single and multiple $\mathrm{THz}$ pulses was investigated $[171,195]$. The observed damage resulted in ablation and through holes as well as delaminations and cracking near the damage threshold. The real-time room-temperature $\mathrm{THz}$ imaging developed by Fan et al. [196] was demonstrated on an aluminum foil by nonlinear frequency up-conversion in a DAST crystal. The results showed higher sensitivity in comparison to a commercial THz camera. Furthermore, analytical and numerical models [197] showed that the rapid heating of aluminum film with thicknesses of several tens of nanometers by the high-energy $\mathrm{THz}$ pulse causes a significant rise of effective electron collision frequency. Consequently, the increase in nonlinear transmission of the thin film as well as noticeable changes in reflectivity can also be observed. All of these recent examples demonstrate a very high potential of intense organic $\mathrm{THz}$ sources for nonlinear THz photonics.

\subsection{General THz Spectroscopy and Imaging}

Most often employed $\mathrm{THz}$ systems for $\mathrm{THz}$ spectroscopy and imaging applications are the so-called THz-TDS (THz time-domain spectroscopy) systems, which are based on optical rectification and can simultaneously measure the amplitude and the phase of the $\mathrm{THz}$ electric field and therefore, for example, the absorption coefficient and the refractive index in the THz range. THz-TDS systems can be also used for non-destructive sample-thickness or coating-thickness measurements [198]. One of the additional features of THz-TDS systems is the possibility of terahertz pulsed imaging. Terahertz pulsed imaging can be performed in transmission or in reflection system geometry by raster-scanning of the sample at the focal plane of a THz-TDS system. In the time-domain, terahertz pulsed imaging can provide 2D amplitude images based on the maximum amplitude or the peak-to-peak amplitude of the transmitted (or reflected) THz electric field as well as 2D phase images by considering the arrival time of the waveform. THz spectroscopic images are obtained by the Fourier transform of time-domain signals at each sample position. Spectroscopic THz imaging and spatial distribution mapping provide visualization and localization of individual chemical compounds within heterogeneous samples, e.g., pharmaceutical pellets. Thus, the complete spectroscopic information about each pixel is obtained, which allows besides detection also identification as well as classification of materials. The chemical analysis is possible at frequencies higher than $500 \mathrm{GHz}$ [199], where $\mathrm{THz}$ spectral responses express significant characteristics that can be assigned to inter and intramolecular vibrations of individual chemical substances. Additionally, data of spectroscopic $\mathrm{THz}$ imaging can be further enriched by the use of different chemometrics methods. Among them, the most used is principal component analysis, where a series of spectra are simultaneously compared through measuring the covariance [200]. The method is also known as chemical mapping and is often applied in the pharmaceutical industry [201,202].

There have been already many examples of broadband $\mathrm{THz}$ spectroscopy measured with $\mathrm{THz}$ system based on organic NLO crystals. For example, optical properties in the $\mathrm{THz}$ range of organic NLO crystals themselves as those presented in Figure 8b and many other crystals have been measured by organic-based THz-TDS systems $[53,83,91,109,126,129]$. The refractive index and absorption coefficients have been also measured using $\mathrm{THz}$ systems based on difference-frequency generation in organic crystals $[127,169]$.

A system based on organic NLO crystal DSTMS as a THz emitter and a photoconductive antenna as a $\mathrm{THz}$ receiver operating in the $0.2-8 \mathrm{THz}$ range [79] was used for measuring a lactose film and two metamaterials, U-shape split-ring resonators and rectangle hole arrays. The obtained results coincide with previous results measured by Fourier-transform infrared spectroscopy or inorganic-based $\mathrm{THz}$ systems.

The refractive indices and the absorption of single crystals of aluminum nitride were measured in the 1-8 THz by THz-TDS based on DSTMS crystals [203]. The selected semiconductor has promising 
optical and optoelectronic properties that can be exploited for optical applications from the far infrared to deep ultraviolet spectral region.

Kawase et al. [204] performed several exploratory experiments with high-resolution time-of-flight $\mathrm{THz}$ tomography using DAST crystal for $\mathrm{THz}$ waves generation. They measured the thickness of Teflon films as a function of actual thickness. THz tomography images of multilayered structures of semiconductor device sample were recorded where GaAs layer was approximately $2 \mu \mathrm{m}$ thick. This method should be valuable for car paint inspection. By using a metallic mesh, they tried to achieve imaging with high sensitivity in the $\mathrm{THz}$ region. They were able to detect $1 \mu \mathrm{m}$ thickness differences in ultrathin PET film which is about 300 times thinner than the wavelength in the $\mathrm{THz}$ frequency range [204].

In the following subsections, we present further examples of $\mathrm{THz}$ spectroscopy and imaging using organic NLO crystals as THz sources in specific industrial sectors.

\subsection{Pharmaceutical and Chemical Industry}

The potential of various $\mathrm{THz}$ systems in the pharmaceutical industry was explored by several studies. For pharmaceutical use, mainly molecular structure, polymorphic forms [205], cocrystals [202], hydrates [206], dehydration processes [207], tablet coating thickness [208], concentration of active pharmaceutical ingredient (API) [178], spatial density distribution $[209,210]$ and impurities [211] were analyzed. THz spectroscopic analysis based on organic NLO crystals was performed to distinguish between the absorption spectra of polymorphs I, II, and V of piroxicam, particularly in the regions below approximately $2 \mathrm{THz}$ and above approximately $3.8 \mathrm{THz}$ [177]. The organic compounds like piroxicam can come in different polymorphic forms that typically differ in their physicochemical properties, which also importantly affect their pharmaceutical performance. Since $\mathrm{THz}$ waves are sensitive to both intermolecular and intramolecular vibrations, THz-TDS can be used to investigate the crystalline state in different chemical species e.g., polymorphism. The same system based on DSTMS crystals was used to analyze hormone-based drug melatonin and its pharmaceutical product Circadin in the $\mathrm{THz}$ frequency range between $1.5 \mathrm{THz}$ and $4.5 \mathrm{THz}$ [178]. In the selected spectral range, melatonin showed characteristic spectral features at $3.21 \mathrm{THz}$ and a weaker one at $4.20 \mathrm{THz}$ allowing a quantitative analysis within the final product. Furthermore, spectroscopic $\mathrm{THz}$ imaging of different concentrations of Circadin and melatonin as an API within pellets was also performed, in order to verify the spatial recognition of different substances [178]. Another study with a DSTMS-based THz-TDS system was performed on paracetamol, where commercial pellets from two manufacturers were compared [212]. In both samples, the same characteristic spectral peaks were observed at $2.1 \mathrm{THz}$, $3.0 \mathrm{THz}, 3.6 \mathrm{THz}$ and $4.5 \mathrm{THz}$. The studies indicated that $\mathrm{THz}$ spectroscopy and imaging using organic $\mathrm{THz}$ sources could be a complementary tool to Raman and Fourier transform infrared spectroscopies, especially in applications of identification and quality control in the pharmaceutical industry.

In different chemical industries ranging from construction materials, paints, glasses, ceramics, adhesives, composites, paper products, polymers and plastics to animal feeds, food ingredients, pharmaceuticals, and cosmetics, powders are main process entering material used as fillers, pigments or additives [213]. Its usefulness and effectiveness in manufacturing processes are defined by several physicochemical properties such as granulation, particle size distribution, hardness, morphology, specific surface area, polymorphism and chemical purity. One of the most abundant raw material calcium carbonate $\left(\mathrm{CaCO}_{3}\right)$ was characterized by $\mathrm{THz}$ spectroscopic and imaging system operating with organic NLO crystals (see Figure 15) [174]. Several parameters such as $\mathrm{CaCO}_{3}$ concentration in a polymer matrix, its granulation, and chemical treatment were analyzed based on the analysis of peak absorbance amplitude at around $3.2 \mathrm{THz}$, the appearance of additional spectral features such as shoulder and small peaks on the first derivative of absorbance spectra, and the frequency position and shift. In the last experiment, the calcite samples were treated by various chemicals. In that case, the frequency position of the characteristic spectral peak at $3.2 \mathrm{THz}$ was shifted to higher frequencies although the powder granulation was the same. The less treated sample, i.e., natural 
calcite, had the most similar peak position as the pure calcite sample, whereas the samples with additives that modified the particle surface have the highest peak shift in comparison to the pure chemical. In addition, $\mathrm{THz}$-TDS and amplitude $\mathrm{THz}$ pulsed imaging were also applied to distinguish $\mathrm{CaCO}_{3}$ from other two calcium compounds, calcium oxide and calcium hydroxide, which appear during the carbonation/decarbonation production process. With spectroscopic $\mathrm{THz}$ imaging, a spatial distribution map was obtained, indicating regions with a various concentration of $\mathrm{CaCO}_{3}$ in a polymer matrix. The results of this study demonstrated that the THz system based on DSTMS crystals has great potential as a rapid analytical tool for qualitative and quantitative analysis of powdered materials.
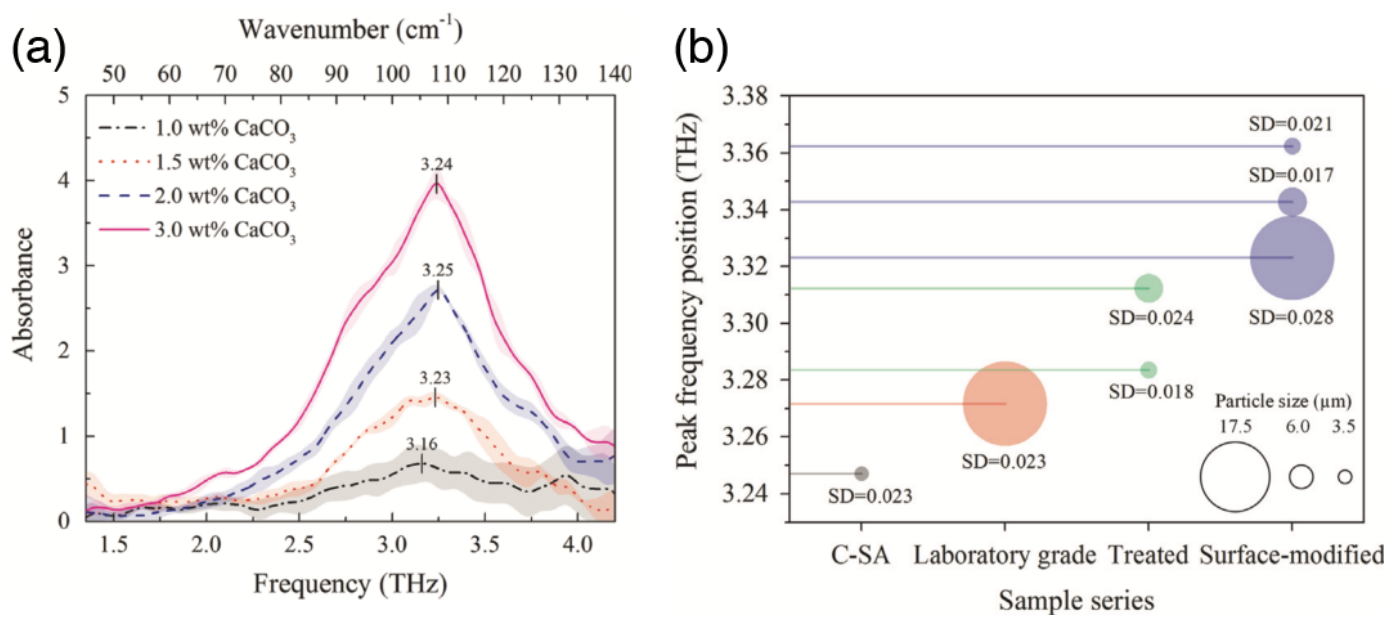

Figure 15. (a) $\mathrm{THz}$ spectra of various concentrations of $\mathrm{CaCO}_{3}$ in a polyethylene (PE) matrix; (b) peak frequency position of pure calcite (C-SA) in comparison with natural calcite of laboratory grade, calcite treated with additives and surface-modified calcite powders. The different size of particles is presented by circles of varying diameter and the error in the peak position is presented by a value of standard deviation (SD) next to each circle [174]. Reprinted from Ref. [174] with permission from Elsevier.

\subsection{Defense and Security}

Terahertz waves can, at least partially and at certain $\mathrm{THz}$ frequencies, penetrate various non-metallic materials such as fabrics, wood, paper, cardboard and plastics. Therefore, they can be used in defense and security screening to uncover the concealed illicit drugs or explosives within packages. Since many dangerous materials have unique spectral fingerprints in the $\mathrm{THz}$ range, it is possible to combine both, detection by $\mathrm{THz}$ imaging and subsequent $\mathrm{THz}$ spectral identification with THz-TDS. Organic crystalline THz sources can be used in the same way as inorganic sources for close-by-inspection of envelopes or suspicious powders hidden under clothes. THz time-domain spectroscopy and imaging system based on DSTMS was used to study the effects of various hiding techniques with different paper and textile barriers based on analyzing the spectral features for drugs and explosive simulants [214]. Besides deposited and impregnated powder samples, mixtures with polyethylene in a form of a pellet were also analyzed hidden behind or within textile or paper barriers simulant. The study confirmed that rapid detection and identification of concealed simulants is possible in the frequency range from $1.5 \mathrm{THz}$ to $4.0 \mathrm{THz}$ by using the spectral peak analysis method and a priori known characteristic spectral features from the database [214]. The DSTMS-based THz system was also used in a comprehensive study where different $\mathrm{THz}$ technologies were compared for detection and identification of 18 explosives in a pellet and powder forms [215]. The selected DSTMS crystals allowed access to higher frequencies compared to the inorganic-based systems (see Figure 16). In some cases, the results showed that minor absorption peaks, bands and shoulders that are visible in the respective frequency region in the Fourier-transform infrared spectroscopy data can appear more prominent in THz-TDS measurements [215]. 


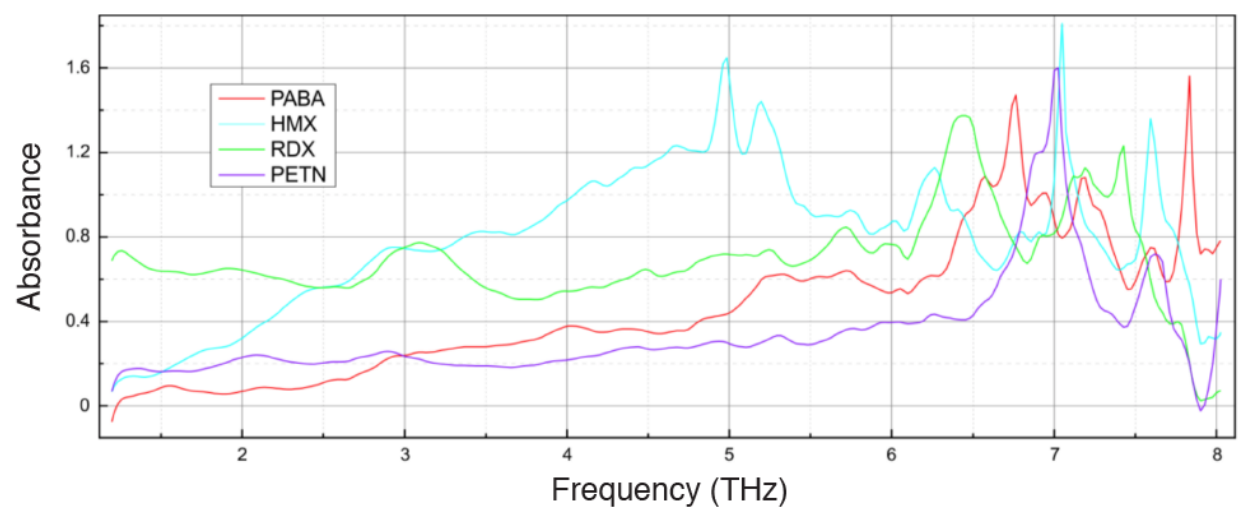

Figure 16. $\mathrm{THz}$ absorption spectra of a reference material PABA (para-aminobenzoic acid) and several explosives (HMX, RDX, PETN) measured with a DSTMS-based THz-TDS system [215]. Copyright 2014 Society of Photo Optical Instrumentation Engineers (SPIE).

\subsection{Food and Agriculture}

$\mathrm{THz}$ waves offer a non-ionizing non-destructive testing method to detect foreign bodies within food products with low water content such as chocolate or powdered eatables, e.g., sugar or flour. In addition, the chemical analysis of food samples such as edible oils is also possible with $\mathrm{THz}$ spectroscopy. THz interaction with food samples provides intramolecular information linked with the existence of certain characteristic bonds indicating the presence of certain molecules or groups of molecules as well as intermolecular information such as hydrogen bonding, alignment of molecules, amorphous or crystalline structure [216]. Because $\mathrm{THz}$ waves are very sensitive to water molecules, THz-TDS is an excellent tool to determine the water content within the food samples such as butter, vegetable oils or flour. A DSTMS-based THz spectroscopic system was used to obtain the absorption coefficient of five different edible oils from different plants in the frequency range from $1.5 \mathrm{THz}$ to $3.5 \mathrm{THz}$ [179]. By using additional chemometrics, also distinguishing between different edible oils was possible. The use of chemicals such as additives and hormones in food production is becoming a critical food safety issue not only for regulatory agencies but also for consumers. Some studies on hormones have already been performed using THz spectroscopy. One such example performed by a DSTMS-based THz system investigated the melatonin [178], which can be used as a dietary supplement. The experiment confirmed that the melatonin has a unique spectral feature at around $3.2 \mathrm{THz}$, which can be used to detect and quantify it within the dietary samples in order to control its authentication. Similarly, other chemicals such as pesticides and antibiotics can also be analyzed. Since pesticides have characteristic spectral response in the THz frequency range, the THz-TDS method can be used to detect pesticides residue in crop samples [216]. Furthermore, qualitative and quantitative analysis of food additives or antibiotics due to veterinary drugs can be performed by THz-TDS.

\subsection{Building and Construction Sector}

Although the reports on possible applications of $\mathrm{THz}$ technology in different sectors constantly increase, the construction and building industry lags behind them. However, several studies demonstrated that $\mathrm{THz}$ spectroscopy and imaging have promising potential and provide many opportunities for applications in construction and building materials characterization [217]. For instance, structural analyses of various thermal building insulation materials such as polymer foams, an aerogel composite, and a microencapsulated phase-change material by using a DSTMS-based THz-TDS and THz pulsed imaging were performed $[175,176,218]$. The insulating materials ensure low thermal conductivity and thermal transmittance to buildings. These characteristics are highly dependent on the thickness and structural properties of materials (e.g., homogeneity, porosity, humidity, density, inclusions, defects, etc.). Studies revealed that the absorption coefficient of polymer foams in the $\mathrm{THz}$ range is inversely related to the thermal conductivity of these foams [175]. The obtained $\mathrm{THz}$ 
amplitude images (see Figure 17) clearly visualized different manufacturing imperfections and internal structures such as beads structure, solvent distribution, air gaps and fibre direction in layers of aerogel blanket $[175,176]$. Based on the observation of additional spectral features during the solidification and liquefying of a phase-change material, $\mathrm{THz}$ spectroscopy was demonstrated to be a suitable method to characterize specific properties related to the freeze-thaw process [175]. This is especially important when phase-change materials are incorporated within a polymer matrix in order to increase the energy efficiency of insulation materials. In addition, a periodical fibrous structure of an aerogel composite was also observable in $\mathrm{THz}$ spectra, where several spectral peaks were present separated by a distance corresponding to the diameter size of fibers [218]. These examples show that THz-TDS and THz pulsed imaging based on organic $\mathrm{THz}$ sources have a potential to become a suitable non-destructive testing method when evaluating the existing building materials as well as when developing novel high-performance fibrous, cellular or granular materials. THz-based methods can provide important construction-material information, especially when combined with other non-destructive testing techniques using ultrasound, microwaves, infrared waves or X-rays.

(a)

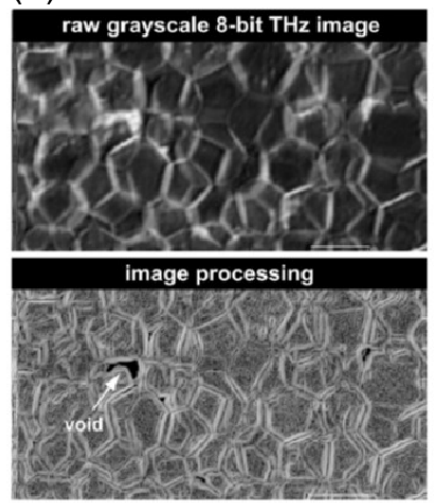

(b)

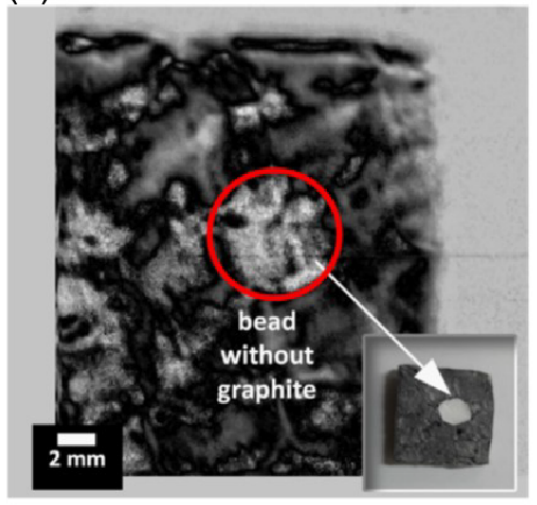

(c)

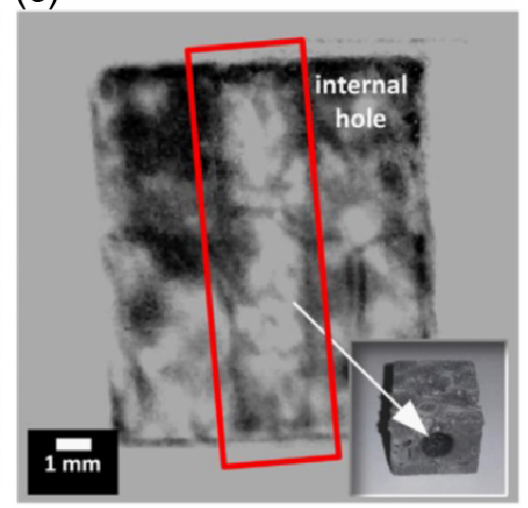

Figure 17. (a) signal processing for particle analysis of $\mathrm{THz}$ image determining the voids within a polymer foam [176]. Detection of imperfections (b) and internal structures (c) by THz imaging [175]. Reprinted from Ref. [176] and Ref. [175] with permission from Elsevier.

\subsection{Future Challenges and Opportunities for Practical Applications}

In the last decade, the rapid development of novel $\mathrm{THz}$ sources and detectors has opened additional possibilities of creating innovative imaging and sensing systems, allowing in situ measurements and real-time analysis of different materials. Among these, THz systems based on organic NLO crystals are very interesting due to their high THz-generation efficiencies, the possibility for high $\mathrm{THz}$ electric fields and a uniquely ultra-broadband nature. We can expect that the recent advances in the field will encourage new research in various disciplines with these systems. Taking into account also other studies performed by inorganic-based $\mathrm{THz}$ systems, we can soon expect some important studies in the field of biological and medical applications [219] because healthy and diseased tissue can be distinguished by $\mathrm{THz}$ waves due to the changes in water content, bacteria presence, structural and molecular changes. Therefore, $\mathrm{THz}$ spectroscopic and imaging analysis of small and macro biomolecules as well as cells and tissues is definitely one of the upcoming research opportunities for organic-crystals based systems. In addition, environmental applications are also an opportunity for laboratories operating with broadband $\mathrm{THz}$ systems such as based on organic NLO crystals. THz waves can penetrate even through rough environmental conditions like aerosol and heavily sooted flames [220]. Since $\mathrm{THz}$ radiation has the ability to gain vibrational information due to inter- and intra-molecular modes and rotational modes, the obtained information can be valuable for gaseous molecules quantification and identification especially in cases of air pollutants detection and atmospheric monitoring. One should also not forget other fields such as art conservation and archeology [221], where $\mathrm{THz}$ technology can play a key role in diagnostics before restoration of 
historical paintings and archaeological objects, and wood products industry [222]. Besides solids, which are already well explored in the $\mathrm{THz}$ region, more attention could also be given to liquids and aqueous solutions as well as gas spectroscopy.

\section{Conclusions and Outlook}

Organic NLO crystals have attracted a considerable interest within the fast-growing field of $\mathrm{THz}$ photonics. In this paper, we reviewed their unique properties that allow on the one hand for achieving state-of-the-art highly-intense $\mathrm{THz}$ fields and on the other hand can cover an ultrabroad $\mathrm{THz}$ frequency range of beyond $20 \mathrm{THz}$. These achievements give organic NLO sources an important advantage for the exciting fundamental and applied studies of $\mathrm{THz}$ light-matter interactions. We presented the basic material properties and requirements for optimized organic NLO crystals for $\mathrm{THz}$ photonics. These materials are composed of highly asymmetric electron-rich molecules with an ultra-fast response to the applied field, packed in a non-centrosymmetric crystalline lattice. Organic NLO crystals feature high long-term temporal as well as the required photochemical stability for high-optical-power applications. Few organic NLO crystals with record-high optical nonlinearities can be successfully grown and can be prepared with sufficient size, orientation, and optical quality for $\mathrm{THz}$ photonics applications. The development of novel materials is however being continued and is highly motivated by the wish of high $\mathrm{THz}$ power over a broad $\mathrm{THz}$ range without any gaps, which still present a challenge due to intrinsic phonon-mode resonances of NLO generation materials themselves. There is also a wish to increase the variety of organic NLO crystals optimized for certain available pump laser sources or compact high-power laser sources being currently developed, considering the optimal pump wavelength range and the desired $\mathrm{THz}$ frequency range. In parallel to the development of novel organic NLO crystals, there has been a significant progress in record high-power and ultra-broadband $\mathrm{THz}$ sources reported during the last few years. Organic crystalline $\mathrm{THz}$ sources became important in the development of the recently emerged field of nonlinear $\mathrm{THz}$ photonics, studying new fundamental aspects of matter enabled by intense $\mathrm{THz}$ waves. We also presented several examples of conventional $\mathrm{THz}$ spectroscopy and $\mathrm{THz}$ imaging based on organic crystalline $\mathrm{THz}$ sources, which show great potential for various scientific and industrial applications. We can therefore expect exciting further progress in research and applications of organic NLO crystals for THz photonics in the near future.

Author Contributions: Coordination, M.J.; Writing and proofreading, M.J., U.P., A.A. and A.Z.

Funding: We acknowledge the support by the Swiss National Science Foundation (SNSF) Grant No. IZKSZ2_162129, InnoSuisse Grant No. 19281.2 PFNM-NM and the Slovenian Research Agency Grant P2-0348.

Conflicts of Interest: The authors declare no conflict of interest.

\section{References}

1. Dhillon, S.S.; Vitiello, M.S.; Linfield, E.H.; Davies, A.G.; Hoffmann, M.C.; Booske, J.; Paoloni, C.; Gensch, M.; Weightman, P.; Williams, G.P.; et al. The 2017 terahertz science and technology roadmap. J. Phys. D Appl. Phys. 2017, 50, 043001. [CrossRef]

2. Du, S.; Yoshida, K.; Zhang, Y.; Hamada, I.; Hirakawa, K. Terahertz dynamics of electron-vibron coupling in single molecules with tunable electrostatic potential. Nat. Photonics 2018, 12, 608. [CrossRef]

3. Bosshard, C. Third-Order Nonlinear Optics in Polar Materials. In Nonlinear Optical Effects and Materials; Günter, P., Ed.; Springer Series in Optical Science; Springer: Berlin/Heidelberg, Germany; New York, NY, USA, 2000; Volume 72, pp. 7-161.

4. Willetts, A.; Rice, J.E.; Burland, D.M.; Shelton, D.P. Problems in the comparison of theoretical and experimental hyperpolarizabilities. J. Chem. Phys. 1992, 97, 7590-7599. [CrossRef]

5. Reis, H. Problems in the comparison of theoretical and experimental hyperpolarizabilities revisited. J. Chem. Phys. 2006, 125, 014506. [CrossRef] [PubMed]

6. Dalton, L.; Gunter, P.; Jazbinsek, M.; Kwon, O.P.; Sullivan, P. Nonlinear Optical Properties of Organic Molecules and Crystals; Cambridge University Press: Cambridge, UK, 2015. 
7. Dalton, L.R.; Sullivan, P.A.; Bale, D.H. Electric Field Poled Organic Electro-optic Materials: State of the Art and Future Prospects. Chem. Rev. 2010, 110, 25-55. [CrossRef] [PubMed]

8. Zyss, J. (Ed.) Molecular Nonlinear Optics: Materials, Physics, Devices; Academic Press: Boston, MA, USA, 1994.

9. Bosshard, C.; Sutter, K.; Prêtre, P.; Hulliger, J.; Flörsheimer, M.; Kaatz, P.; Günter, P. Organic Nonlinear Optical Materials; Gordon and Breach Science Publishers: Amsterdam, The Netherlands, 1995.

10. Bosshard, C.; Gunter, P. Electro-Optic Effects in Organic Molecules and Polymers. In Nonlinear Optics of Organic Molecules and Polymers; Nalwa, H.S., Miyata, S., Eds.; CRC Press: Boca Raton, FL, USA, 1997; p. 391.

11. Bosshard, C.; Bösch, M.; Liakatas, I.; Jäger, M.; Günter, P. Second-Order Nonlinear Optical Organic Materials: Recent Developments. In Nonlinear Optical Effects and Materials; Günter, P., Ed.; Springer Series in Optical Science; Springer: Berlin/Heidelberg, Germany; New York, NY, USA, 2000; Volume 72, pp. 163-300.

12. Kuzyk, M.G. Physical limits on electronic nonlinear molecular susceptibilities. Phys. Rev. Lett. 2000, 85, 1218. [CrossRef] [PubMed]

13. Clays, K.; Coe, B.J. Design strategies versus limiting theory for engineering large second-order nonlinear optical polarizabilities in charged organic molecules. Chem. Mater. 2003, 15, 642. [CrossRef]

14. Zyss, J.; Oudar, J.L. Relations between microscopic and macroscopic lowest-order optical nonlinearities of molecular-crystals with one-dimensional or two-dimensional units. Phys. Rev. A 1982, 26, 2028. [CrossRef]

15. Lee, S.H.; Jazbinsek, M.; Hauri, C.P.; Kwo, O.P. Recent progress in acentric core structures for highly efficient nonlinear optical crystals and their supramolecular interactions and terahertz applications. CrystEngComm 2016, 18, 7180-7203. [CrossRef]

16. Levine, B.F.; Bethea, C.G. Second and third order hyperpolarizabilities of organic molecules. J. Chem. Phys. 1975, 63, 2666-2682. [CrossRef]

17. Goovaerts, E.; Wenseleers, W.E.; Garcia, M.H.; Cross, G.H. Chapter 3-Design and characterization of organic and organometallic molecules for second order nonlinear optics. In Handbook of Advanced Electronic and Photonic Materials and Devices; Nalwa, H.S., Ed.; Academic Press: Burlington, NJ, USA, 2001; pp. 127-191. [CrossRef]

18. Butcher, P.N.; Cotter, D. The Elements of Nonlinear Optics; Cambridge University Press: Cambridge, UK, 1990.

19. Boyd, R.W. Nonlinear Optics; Elsevier: Amsterdam, The Netherlands, 2003.

20. Sutherland, R.L. Handbook of Nonlinear Optics; Dekker: New York, NY, USA, 2003.

21. Bosshard, C.; Spreiter, R.; Degiorgi, L.; Gunter, P. Infrared and Raman spectroscopy of the organic crystal DAST: Polarization dependence and contribution of molecular vibrations to the linear electro-optic effect. Phys. Rev. B 2002, 66, 205107. [CrossRef]

22. Jazbinsek, M.; Mutter, L.; Guenter, P. Photonic Applications With the Organic Nonlinear Optical Crystal DAST. IEEE J. Sel. Top. Quantum Electron. 2008, 14, 1298-1311. [CrossRef]

23. Pan, F.; Knopfle, G.; Bosshard, C.; Follonier, S.; Spreiter, R.; Wong, M.S.; Gunter, P. Electro-optic properties of the organic salt 4-N,N-dimethylamino-4'-N'-methyl-stilbazolium tosylate. Appl. Phys. Lett. 1996, 69, $13-15$. [CrossRef]

24. Jazbinsek, M.; Zgonik, M. Material tensor parameters of LiNbO3 relevant for electro- and elasto-optics. Appl. Phys. B 2002, 74, 407-414.

25. Jazbinsek, M.; Günter, P. Organic Electro-Optic Crystal Modulators. In Broadband Optical Modulators; CRC Press: Boca Raton, FL, USA, 2011; pp. 281-306. [CrossRef]

26. Leuthold, J.; Koos, C.; Freude, W.; Alloatti, L.; Palmer, R.; Korn, D.; Pfeifle, J.; Lauermann, M.; Dinu, R.; Wehrli, S.; et al. Silicon-Organic Hybrid Electro-Optical Devices. IEEE J. Sel. Top. Quantum Electron. 2013, 19, 3401413. [CrossRef]

27. Zheng, X.M.; McLaughlin, C.V.; Cunningham, P.; Hayden, L.M. Organic broadband terahertz sources and sensors. J. Nanoelectron. Optoelectron. 2007, 2, 58-76. [CrossRef]

28. Lee, S.H.; Yoo, B.W.; Jazbinsek, M.; Kang, B.J.; Rotermund, F.; Kwon, O.P. Organic ionic electro-optic crystals grown by specific interactions on templates for THz wave photonics. Crystengcomm 2015, 17, 4781-4786. [CrossRef]

29. Rezzonico, D.; Kwon, S.; Figi, H.; Kwon, O.; Jazbinsek, M.; Gunter, P. Photochemical stability of nonlinear optical chromophores in polymeric and crystalline materials. J. Chem. Phys. 2008, 128, 124713:1-124713:6. [CrossRef] [PubMed]

30. Liu, X.; Yang, Z.; Wang, D.; Cao, H. Molecular Structures and Second-Order Nonlinear Optical Properties of Ionic Organic Crystal Materials. Crystals 2016, 6, 158. [CrossRef] 
31. Cole, J.M.; Lin, T.C.; Edwards, A.J.; Piltz, R.O.; Depotter, G.; Clays, K.; Lee, S.C.; Kwon, O.P. Concerted Mitigation of $\mathrm{O} \cdots \mathrm{H}$ and $\mathrm{C}(\pi) \cdots \mathrm{H}$ Interactions Prospects Sixfold Gain in Optical Non linearity of Ionic Stilbazolium Derivatives. ACS Appl. Mater. Interfaces 2015, 7, 4693-4698. [CrossRef] [PubMed]

32. Yang, Z.; Jazbinsek, M.; Ruiz, B.; Aravazhi, S.; Gramlich, V.; Gunter, P. Molecular engineering of stilbazolium derivatives for second-order nonlinear optics. Chem. Mater. 2007, 19, 3512-3518. [CrossRef]

33. Oudar, J.L.; Chemla, D.S. Hyperpolarizabilities of nitroanilines and their relations to excited-state dipole-moment. J. Chem. Phys. 1977, 66, 2664. [CrossRef]

34. Wang, C.H. Effects of dephasing and vibronic structure on the first hyperpolarizability of strongly charge-transfer molecules. J. Chem. Phys. 2000, 112, 1917-1924. [CrossRef]

35. Berkovic, G.; Meshulam, G.; Kotler, Z. Measurement and analysis of molecular hyperpolarizability in the two-photon resonance regime. J. Chem. Phys. 2000, 112, 3997-4003. [CrossRef]

36. Campo, J.; Wenseleers, W.; Goovaerts, E.; Szablewski, M.; Cross, G.H. Accurate Determination and Modeling of the Dispersion of the First Hyperpolarizability of an Efficient Zwitterionic Nonlinear Optical Chromophore by Tunable Wavelength Hyper-Rayleigh Scattering. J. Phys. Chem. C 2008, 112, 287-296. [CrossRef]

37. Maker, P.D.; Terhune, R.W.; Nisenoff, M.; Savage, C.M. Effects of Dispersion and Focusing on the Production of Optical Harmonics. Phys. Rev. Lett. 1962, 8, 21-22. [CrossRef]

38. Herman, W.N.; Hayden, L.M. Maker Fringes Revisited-2nd-harmonic Generation from Birefringent or Absorbing Materials. J. Opt. Soc. Am. B-Opt. Phys. 1995, 12, 416-427. [CrossRef]

39. Hunziker, C.; Kwon, S.J.; Figi, H.; Juvalta, F.; Kwon, O.P.; Jazbinsek, M.; Gunter, P. Configurationally locked, phenolic polyene organic crystal 2-3-(4-hydroxystyryl)-5,5-dimethylcyclohex-2-enylidenemalononitrile: Linear and nonlinear optical properties. J. Opt. Soc. Am. B 2008, 25, 1678-1683. [CrossRef]

40. Kurtz, S.K.; Perry, T.T. A powder technique for evaluation of nonlinear optical materials. J. Appl. Phys. 1968, 39, 3798. [CrossRef]

41. Marder, S.R.; Perry, J.W.; Schaefer, W.P. Synthesis Of Organic Salts With Large 2nd-Order Optical Nonlinearities. Science 1989, 245, 626-628. [CrossRef] [PubMed]

42. Nakanishi, H.; Matsuda, H.; Okada, S.; Kato, M. Organic and polymeric ion-complexes for nonlinear optics. In Materials Research Society International Meeting on Advanced Materials; Doyama, M., Somiya, S., Chang, R.P.H., Eds.; Materials Research Society: Pittsburgh, PA, USA, 1989; pp. 97-104.

43. Ruiz, B.; Jazbinsek, M.; Gunter, P. Crystal Growth of DAST. Cryst. Growth Des. 2008, 8, 4173-4184. [CrossRef]

44. Yang, Z.; Mutter, L.; Stillhart, M.; Ruiz, B.; Aravazhi, S.; Jazbinsek, M.; Schneider, A.; Gramlich, V.; Guenter, P. Large-size bulk and thin-film stilbazolium-salt single crystals for nonlinear optics and $\mathrm{THz}$ generation. Adv. Funct. Mater. 2007, 17, 2018-2023. [CrossRef]

45. Kwon, O.P.; Kwon, S.J.; Jazbinsek, M.; Brunner, F.D.J.; Seo, J.I.; Hunziker, C.; Schneider, A.; Yun, H.; Lee, Y.S.; Gunter, P. Organic Phenolic Configurationally Locked Polyene Single Crystals for Electro-optic and Terahertz Wave Applications. Adv. Funct. Mater. 2008, 18, 3242-3250. [CrossRef]

46. Kwon, S.J.; Jazbinsek, M.; Kwon, O.P.; Guenter, P. Crystal Growth and Morphology Control of OH1 Organic Electrooptic Crystals. Cryst. Growth Des. 2010, 10, 1552-1558. [CrossRef]

47. Jeong, J.H.; Kang, B.J.; Kim, J.S.; Jazbinsek, M.; Lee, S.H.; Lee, S.C.; Baek, I.H.; Yun, H.; Kim, J.; Lee, Y.S.; et al. High-power Broadband Organic THz Generator. Sci. Rep. 2013, 3, 3200. [CrossRef] [PubMed]

48. Pan, F.; Wong, M.S.; Bosshard, C.; Gunter, P. Crystal growth and characterization of the organic salt 4-N,N-dimethylamino-4'-N'-methyl-stilbazolium tosylate (DAST). Adv. Mater. 1996, 8, 592-595. [CrossRef]

49. Marder, S.R.; Perry, J.W.; Yakymyshyn, C.P. Organic Salts With Large 2nd-Order Optical Nonlinearities. Chem. Mater. 1994, 6, 1137-1147. [CrossRef]

50. Kim, P.J.; Jeong, J.H.; Jazbinsek, M.; Kwon, S.J.; Yun, H.; Kim, J.T.; Lee, Y.S.; Baek, I.H.; Rotermund, F.; Gunter, P.; et al. Acentric nonlinear optical N-benzyl stilbazolium crystals with high environmental stability and enhanced molecular nonlinearity in solid state. CrystEngComm 2011, 13, 444-451. [CrossRef]

51. Meier, U.; Bosch, M.; Bosshard, C.; Pan, F.; Gunter, P. Parametric interactions in the organic salt 4-N,N-dimethylamino-4'-N'-methyl-stilbazolium tosylate at telecommunication wavelengths. J. Appl. Phys. 1998, 83, 3486. [CrossRef]

52. Mutter, L.; Brunner, F.D.J.; Yang, Z.; Jazbinsek, M.; Guenter, P. Linear and nonlinear optical properties of the organic crystal DSTMS. J. Opt. Soc. Am. B-Opt. Phys. 2007, 24, 2556-2561. [CrossRef]

53. Brunner, F.D.J.; Lee, S.H.; Kwon, O.P.; Feurer, T. THz generation by optical rectification of near-infrared laser pulses in the organic nonlinear optical crystal HMQ-TMS. Opt. Mater. Express 2014, 4, 1586-1592. [CrossRef] 
54. Kang, B.J.; Lee, S.H.; Kim, W.T.; Lee, S.C.; Lee, K.; Benacchio, G.; Montemezzani, G.; Jazbinsek, M.; Kwon, O.P.; Rotermund, F. New Class of Efficient Terahertz Generators: Effective Terahertz Spectral Filling by Complementary Tandem Configuration of Nonlinear Organic Crystals. Adv. Funct. Mater. 2018, 28, 1707195. [CrossRef]

55. Hashimoto, H.; Okada, Y.; Fujimura, H.; Morioka, M.; Sugihara, O.; Okamoto, N.; Matsushima, R. Second-harmonic generation from single crystals of N-substituted 4-nitroanilines. Jpn. J. Appl. Phys. Part 1 1997, 36, 6754-6760. [CrossRef]

56. Fujiwara, M.; Maruyama, M.; Sugisaki, M.; Takahashi, H.; Aoshima, S.I.; Cogdell, R.J.; Hashimoto, H. Determination of the d-tensor components of a single crystal of N-benzyl-2-methyl-4-nitroaniline. Jpn. J. Appl. Phys. Part 1 2007, 46, 1528-1530. [CrossRef]

57. Piela, K.; Turowska-Tyrk, I.; Drozd, M.; Szostak, M.M. Polymorphism and cold crystallization in optically nonlinear N-benzyl-2-methyl-4-nitroaniline crystal studied by X-ray diffraction, calorimetry and Raman spectroscopy. J. Mol. Struct. 2011, 991, 42-49. [CrossRef]

58. Wang, Z.; Sun, W.; Chen, A.; Kosilkin, I.; Bale, D.; Dalton, L.R. Organic electro-optic thin films by simultaneous vacuum deposition and laser-assisted poling. Opt. Lett. 2011, 36, 2853-2855. [CrossRef] [PubMed]

59. Bernerd, C.; Segonds, P.; Debray, J.; Notake, T.; Koyama, M.; Minamide, H.; Ito, H.; Boulanger, B. Quadratic nonlinear optical properties of the organic $N$-benzyl-2-methyl-4-nitroaniline (BNA) biaxial crystal. Opt. Lett. 2018, 43, 1818-1821. [CrossRef] [PubMed]

60. Glavcheva, Z.; Umezawa, H.; Mineno, Y.; Odani, T.; Okada, S.; Ikeda, S.; Taniuchi, T.; Nakanishi, H. Synthesis and properties of i-methyl-4-2-[4-(dimethylamino)phenyl]ethenylpyridinium p-toluenesulfonate derivatives with isomorphous crystal structure. Jpn. J. Appl. Phys. Part 1 2005, 44, 5231-5235. [CrossRef]

61. Coe, B.J.; Harris, J.A.; Asselberghs, I.; Clays, K.; Olbrechts, G.; Persoons, A.; Hupp, J.T.; Johnson, R.C.; Coles, S.J.; Hursthouse, M.B.; et al. Quadratic nonlinear optical properties of N-aryl stilbazolium dyes. Adv. Funct. Mater. 2002, 12, 110. [CrossRef]

62. Figi, H.; Mutter, L.; Hunziker, C.; Jazbinsek, M.; Gunter, P.; Coe, B.J. Extremely large nonresonant second-order nonlinear optical response in crystals of the stilbazolium salt DAPSH. J. Opt. Soc. Am. B 2008, 25, 1786-1793. [CrossRef]

63. Kim, P.J.; Jeong, J.H.; Jazbinsek, M.; Choi, S.B.; Baek, I.H.; Kim, J.T.; Rotermund, F.; Yun, H.; Lee, Y.S.; Gunter, P.; et al. Highly Efficient Organic THz Generator Pumped at Near-Infrared: Quinolinium Single Crystals. Adv. Funct. Mater. 2012, 22, 200-209. [CrossRef]

64. Yin, J.; Li, L.; Yang, Z.; Jazbinsek, M.; Tao, X.; Guenter, P.; Yang, H. A new stilbazolium salt with perfectly

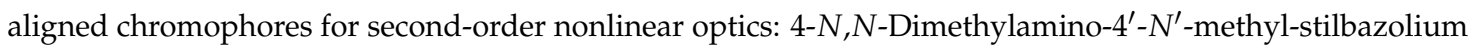
3-carboxy-4-hydroxybenzenesulfonate. Dyes Pigments 2012, 94, 120-126. [CrossRef]

65. Chen, H.; Ma, O.; Zhou, Y.; Yang, Z.; Jazbinsek, M.; Bian, Y.; Ye, N.; Wang, D.; Cao, H.; He, W. Engineering of Organic Chromophores with Large Second-Order Optical Nonlinearity and Superior Crystal Growth Ability. Cryst. Growth Des. 2015, 15, 5560-5567. [CrossRef]

66. Ogawa, J.; Okada, S.; Glavcheva, Z.; Nakanishi, H. Preparation, properties and structures of 1-methyl-4-2-[4(dimethylamino)phenyl]ethenylpyridinium crystals with various counter anions. J. Cryst. Growth 2008, 310, 836-842. [CrossRef]

67. Lee, S.H.; Lu, J.; Lee, S.J.; Han, J.H.; Jeong, C.U.; Lee, S.C.; Li, X.; Jazbinsek, M.; Yoon, W.; Yun, H.; et al. Benzothiazolium Single Crystals: A New Class of Nonlinear Optical Crystals with Efficient THz Wave Generation. Adv. Mater. 2017, 29. [CrossRef] [PubMed]

68. Lee, S.H.; Lee, G.H.; Lee, K.H.; Jazbinsek, M.; Kang, B.J.; Rotermund, F.; Kwon, O.P. In Situ Tailor-Made Additives for Molecular Crystals: A Simple Route to Morphological Crystal Engineering. Cryst. Growth Des. 2016, 16, 3555-3561. [CrossRef]

69. Kim, J.Y.; Lee, S.H.; Choi, I.Y.; Choi, J.Y.; Lee, S.C.; Jazbinsek, M.; Kim, W.S.; Kwak, S.K.; Huh, Y.S.; Kang, J.W.; et al. Stereoselective Inhibitors Based on Nonpolar Hydrocarbons for Polar Organic Crystals. Cryst. Growth Des. 2016, 16, 6514-6521. [CrossRef]

70. Choi, J.Y.; Lee, S.J.; Lee, S.C.; Jeong, C.U.; Jazbinsek, M.; Yun, H.; Kang, B.J.; Rotermundd, F.; Kwon, O.P. Quinolinium single crystals with a high optical nonlinearity and unusual out-of-plane polar axis. J. Mater. Chem. C 2017, 5, 12602-12609. [CrossRef] 
71. Lee, S.H.; Lee, S.J.; Jazbinsek, M.; Kang, B.J.; Rotermund, F.; Kwon, O.P. Electro-optic crystals grown in confined geometry with optimal crystal characteristics for THz photonic applications. CrystEngComm 2016, 18, 7311-7318. [CrossRef]

72. Stillhart, M.; Schneider, A.; Guenter, P. Optical properties of 4-N,N-dimethylamino- $4^{\prime}-N^{\prime}$-methyl-stilbazolium 2,4,6-trimethylbenzenesulfonate crystals at terahertz frequencies. J. Opt. Soc. Am. B-Opt. Phys. 2008, 25, 1914-1919. [CrossRef]

73. Liu, P.; Xu, D.; Li, Y.; Zhang, X.; Wang, Y.; Yao, J.; Wu, Y. Widely tunable and monochromatic terahertz difference frequency generation with organic crystal DSTMS. EPL 2014, 106, 60001. [CrossRef]

74. Vicario, C.; Jazbinsek, M.; Ovchinnikov, A.V.; Chefonov, O.V.; Ashitkov, S.I.; Agranat, M.B.; Hauri, C.P. High efficiency THz generation in DSTMS, DAST and OH1 pumped by Cr:forsterite laser. Opt. Express 2015, 23, 4573-4580. [CrossRef] [PubMed]

75. Monoszlai, B.; Vicario, C.; Jazbinsek, M.; Hauri, C.P. High-energy terahertz pulses from organic crystals: DAST and DSTMS pumped at Ti:sapphire wavelength. Opt. Lett. 2013, 38, 5106-5109. [CrossRef] [PubMed]

76. Somma, C.; Folpini, G.; Gupta, J.; Reimann, K.; Woerner, M.; Elsaesser, T. Ultra-broadband terahertz pulses generated in the organic crystal DSTMS. Opt. Lett. 2015, 40, 3404-3407. [CrossRef] [PubMed]

77. Finneran, I.A.; Welsch, R.; Allodi, M.A.; Miller, T.F.; Blake, G.A. Coherent two-dimensional terahertz-terahertzRaman spectroscopy. Proc. Natl. Acad. Sci. USA 2016, 113, 6857-6861. [CrossRef] [PubMed]

78. Liu, B.; Bromberger, H.; Cartella, A.; Gebert, T.; Foerst, M.; Cavalleri, A. Generation of narrowband, high-intensity, carrier-envelope phase-stable pulses tunable between 4 and $18 \mathrm{THz}$. Opt. Lett. 2017, 42, 129-131. [CrossRef] [PubMed]

79. Zhang, Y.; Zhang, X.; Li, S.; Gu, J.; Li, Y.; Tian, Z.; Ouyang, C.; He, M.; Han, J.; Zhang, W. A Broadband THz-TDS System Based on DSTMS Emitter and LTG InGaAs/InAlAs Photoconductive Antenna Detector. Sci. Rep. 2016, 6. [CrossRef] [PubMed]

80. Ilyakov, I.E.; Kitaeva, G.K.; Shishkin, B.V.; Akhmedzhanov, R.A. The use of DSTMS crystal for broadband terahertz electro-optic sampling based on laser pulse amplitude changes. Laser Phys. Lett. 2018, 15, 125401. [CrossRef]

81. Chai, X.; Ropagnol, X.; Ovchinnikov, A.; Chefonov, O.; Ushakov, A.; Garcia-Rosas, C.M.; Isgandarov, E.; Agranat, M.; Ozaki, T.; Savelév, A. Observation of crossover from intraband to interband nonlinear terahertz optics. Opt. Lett. 2018, 43, 5463. [CrossRef] [PubMed]

82. Lu, J.; Hwang, H.Y.; Li, X.; Lee, S.H.; Kwon, O.P.; Nelson, K.A. Tunable multi-cycle THz generation in organic crystal HMQ-TMS. Opt. Express 2015, 23, 22723-22729. [CrossRef] [PubMed]

83. Vicario, C.; Monoszlai, B.; Jazbinsek, M.; Lee, S.H.; Kwon, O.P.; Hauri, C.P. Intense, carrier frequency and bandwidth tunable quasi single-cycle pulses from an organic emitter covering the Terahertz frequency gap. Sci. Rep. 2015, 5, 14394. [CrossRef] [PubMed]

84. Rovere, A.; Jeong, Y.G.; Piccoli, R.; Lee, S.H.; Lee, S.C.; Kwon, O.P.; Jazbinsek, M.; Morandotti, R.; Razzari, L. Generation of high-field terahertz pulses in an HMQ-TMS organic crystal pumped by an ytterbium laser at $1030 \mathrm{~nm}$. Opt. Express 2018, 26, 2509-2516. [CrossRef] [PubMed]

85. Brunner, F.D.J.; Kwon, O.P.; Kwon, S.J.; Jazbinsek, M.; Schneider, A.; Gunter, P. A hydrogen-bonded organic nonlinear optical crystal for high-efficiency terahertz generation and detection. Opt. Express 2008, 16, 16496-16508. [CrossRef] [PubMed]

86. Kwon, S.J.; Hunziker, C.; Kwon, O.P.; Jazbinsek, M.; Gunter, P. Large-Area Organic Electro-optic Single Crystalline Thin Films Grown by Evaporation-Induced Local Supersaturation with Surface Interactions. Cryst. Growth Des. 2009, 9, 2512-2516. [CrossRef]

87. Uchida, H.; Yamazaki, R.; Oota, K.; Okimura, K.; Minami, T.; Takeya, K.; Kawase, K. Organic Nonlinear Optical Single-Crystalline Thin Film Grown by Physical Vapor Deposition for Terahertz Generation. Cryst. Growth Des. 2018, 18, 4029-4036. [CrossRef]

88. Ruchert, C.; Vicario, C.; Hauri, C.P. Scaling submillimeter single-cycle transients toward megavolts per centimeter field strength via optical rectification in the organic crystal OH1. Opt. Lett. 2012, 37, 899-901. [CrossRef] [PubMed]

89. Uchida, H.; Tripathi, S.R.; Suizu, K.; Shibuya, T.; Osumi, T.; Kawase, K. Widely tunable broadband terahertz radiation generation using a configurationally locked polyene 2-[3-(4-hydroxystyryl)-5,5-dimethylcyclohex2-enylidene] malononitrile crystal via difference frequency generation. Appl. Phys. B-Lasers Opt. 2013, 111, 489-493. [CrossRef] 
90. Stepanov, A.G.; Ruchert, C.; Levallois, J.; Erny, C.; Hauri, C.P. Generation of broadband THz pulses in organic crystal $\mathrm{OH} 1$ at room temperature and $10 \mathrm{~K}$. Opt. Mater. Express 2014, 4, 870-875. [CrossRef]

91. Majkic, A.; Zgonik, M.; Petelin, A.; Jazbinsek, M.; Ruiz, B.; Medrano, C.; Guenter, P. Terahertz source at 9.4 THz based on a dual-wavelength infrared laser and quasi-phase matching in organic crystals $\mathrm{OH} 1$. Appl. Phys. Lett. 2014, 105, 141115. [CrossRef]

92. Li, Y.; Wu, Z.; Zhang, X.; Wang, L.; Zhang, J.; Wu, Y. Crystal growth and terahertz wave generation of organic NLO crystals: OH1. J. Cryst. Growth 2014, 402, 53-59. [CrossRef]

93. Vicario, C.; Ruchert, C.; Hauri, C.P. High field broadband THz generation in organic materials. J. Mod. Opt. 2015, 62, 1480-1485. [CrossRef]

94. Shalaby, M.; Hauri, C.P. Demonstration of a low-frequency three-dimensional terahertz bullet with extreme brightness. Nat. Commun. 2015, 6, 5976. [CrossRef] [PubMed]

95. Brenier, A. Two-frequency pulsed YLiF4:Nd lasing out of the principal axes and THz generation. Opt. Lett. 2015, 40, 4496-4499. [CrossRef] [PubMed]

96. Ovchinnikov, A.V.; Chefonov, O.V.; Molchanov, V.Y.; Yushkov, K.B.; Vicario, C.; Hauri, C. Generation of frequency-tunable pulsed terahertz radiation by a Cr: Forsterite laser system with an acoustooptical control of the pulse temporal profile. Quantum Electron. 2016, 46, 1149-1153. [CrossRef]

97. Liu, P.; Zhang, X.; Yan, C.; Xu, D.; Li, Y.; Shi, W.; Zhang, G.; Zhang, X.; Yao, J.; Wu, Y. Widely tunable and monochromatic terahertz difference frequency generation with organic crystal 2-(3-(4-hydroxystyryl)-5, 5-dime-thylcyclohex-2-enylidene) malononitrile. Appl. Phys. Lett. 2016, 108, 011104. [CrossRef]

98. Uchida, H.; Oota, K.; Okimura, K.; Kawase, K.; Takeya, K. Single-Cycle Terahertz Pulse Generation from OH1 Crystal via Cherenkov Phase Matching. J. Infrared Millim. Terahertz Waves 2018, 39, 509-513. [CrossRef]

99. Chemla, D.S.; Zyss, J. Organic Nonlinear Optics: Molecules, Polymers and Crystals; Academic Press: Orlando, FL, USA, 1987.

100. Korn, D.; Jazbinsek, M.; Palmer, R.; Baier, M.; Alloatti, L.; Yu, H.; Bogaerts, W.; Lepage, G.; Verheyen, P.; Absil, P.; et al. Electro-Optic Organic Crystal Silicon High-Speed Modulator. IEEE Photonics J. 2014, 6, 2700109. [CrossRef]

101. Kuroyanagi, K.; Fujiwara, M.; Hashimoto, H.; Takahashi, H.; Aoshima, S.I.; Tsuchiya, Y. All organic terahertz electromagnetic wave emission and detection using highly purified $\mathrm{N}$-benzyl-2-methyl-4-nitroaniline crystals. Jpn. J. Appl. Phys. Part 1-Regul. Pap. Brief Commun. Rev. Pap. 2006, 45, 4068-4073. [CrossRef]

102. Miyamoto, K.; Minamide, H.; Fujiwara, M.; Hashimoto, H.; Ito, H. Widely tunable terahertz-wave generation using an N-benzyl-2-methyl-4-nitro aniline crystal. Opt. Lett. 2008, 33, 252-254. [CrossRef] [PubMed]

103. Miyamoto, K.; Ohno, S.; Fujiwara, M.; Minamide, H.; Hashimoto, H.; Ito, H. Optimized terahertz-wave generation using BNA-DFG. Opt. Express 2009, 17, 14832-14838. [CrossRef] [PubMed]

104. Iwaszczuk, K.; Cooke, D.G.; Fujiwara, M.; Hashimoto, H.; Jepsen, P.U. Simultaneous reference and differential waveform acquisition in time-resolved terahertz spectroscopy. Opt. Express 2009, 17, 21969-21976. [CrossRef] [PubMed]

105. Notake, T.; Nawata, K.; Kawamata, H.; Matsukawa, T.; Minamide, H. Solution growth of high-quality organic N-benzyl-2-methyl-4-nitroaniline crystal for ultra-wideband tunable DFG-THz source. Opt. Mater. Express 2012, 2, 119-125. [CrossRef]

106. Notake, T.; Nawata, K.; Kawamata, H.; Matsukawa, T.; Qi, F.; Minamide, H. Development of an ultra-widely tunable DFG-THz source with switching between organic nonlinear crystals pumped with a dual-wavelength BBO optical parametric oscillator. Opt. Express 2012, 20, 25850-25857. [CrossRef] [PubMed]

107. Shalaby, M.; Vicario, C.; Thirupugalmani, K.; Brahadeeswaran, S.; Hauri, C.P. Intense THz source based on BNA organic crystal pumped at Ti:sapphire wavelength. Opt. Lett. 2016, 41, 1777-1780. [CrossRef] 
[CrossRef] [PubMed]

108. Thirupugalmani, K.; Venkatesh, M.; Karthick, S.; Maurya, K.K.; Vijayan, N.; Chaudhary, A.K.; Brahadeeswaran, S. Influence of polar solvents on growth of potentially NLO active organic single crystals of N-benzyl-2-methyl-4-nitroaniline and their efficiency in terahertz generation. Crystengcomm 2017, 19, 2623-2631. [CrossRef]

109. Brunner, F.D.J.; Schneider, A.; Guenter, P. Velocity-matched terahertz generation by optical rectification in an organic nonlinear optical crystal using a Ti:sapphire laser. Appl. Phys. Lett. 2009, 94, 061119. [CrossRef]

110. Brunner, F.D.J. Generation and detection of terahertz pulses in the organic crystals OH1 and COANP. Ph.D. Thesis, ETH Zurich, Zürich, Switzerland, 2009. [CrossRef]

111. Ravi, K.; Huang, W.R.; Carbajo, S.; Nanni, E.A.; Schimpf, D.N.; Ippen, E.P.; Kärtner, F.X. Theory of terahertz generation by optical rectification using tilted-pulse-fronts. Opt. Express 2015, 23, 5253. [CrossRef] [PubMed]

112. Schneider, A.; Neis, M.; Stillhart, M.; Ruiz, B.; Khan, R.U.A.; Gunter, P. Generation of terahertz pulses through optical rectification in organic DAST crystals: theory and experiment. J. Opt. Soc. Am. B 2006, 23, 1822-1835. [CrossRef]

113. Schneider, A. Theory of terahertz pulse generation through optical rectification in a nonlinear optical material with a finite size. Phys. Rev. A 2010, 82, 033825. [CrossRef]

114. Weling, A.S.; Auston, D.H. Novel sources and detectors for coherent tunable narrow-band terahertz radiation in free space. J. Opt. Soc. Am. B 1996, 13, 2783. [CrossRef]

115. Vicario, C.; Ovchinnikov, A.V.; Chefonov, O.V.; Hauri, C.P. Multi-octave spectrally tunable strong-field Terahertz laser. arXiv 2016, arXiv:1608.05319.

116. Burford, N.M.; El-Shenawee, M.O. Review of terahertz photoconductive antenna technology. Opt. Eng. 2017, 56, 010901. [CrossRef]

117. Dai, J.; Xie, X.; Zhang, X.C. Detection of Broadband Terahertz Waves with a Laser-Induced Plasma in Gases. Phys. Rev. Lett. 2006, 97. [CrossRef] [PubMed]

118. Clough, B.; Liu, J.; Zhang, X.C. “All air-plasma” terahertz spectroscopy. Opt. Lett. 2011, 36, 2399. [CrossRef] [PubMed]

119. Wu, Q.; Zhang, X.C. Ultrafast electro-optic field sensors. Appl. Phys. Lett. 1996, 68, 1604-1606. [CrossRef]

120. Han, P.Y.; Tani, M.; Pan, F.; Zhang, X.C. Use of the organic crystal DAST for terahertz beam applications. Opt. Lett. 2000, 25, 675-677. [CrossRef] [PubMed]

121. Schneider, A.; Biaggio, I.; Gunter, P. Terahertz-induced lensing and its use for the detection of terahertz pulses in a birefringent crystal. Appl. Phys. Lett. 2004, 84, 2229-2231. [CrossRef]

122. Martin, M.; Mangeney, J.; Crozat, P.; Mounaix, P. Optical phase detection in a 4-N,N-dimethylamino$4^{\prime}-N^{\prime}$-methyl-stilbazolium tosylate crystal for terahertz time domain spectroscopy system at $1.55 \mu \mathrm{m}$ wavelength. Appl. Phys. Lett. 2010, 97, 111112. [CrossRef]

123. Ilyakov, I.E.; Kitaeva, G.K.; Shishkin, B.V.; Akhmedzhanov, R.A. Laser pulse amplitude changes induced by terahertz waves under linear electro-optic effect. Appl. Phys. Lett. 2014, 104, 151107. [CrossRef]

124. Schneider, A.; Gunter, P. Coherent detection of terahertz pulses based on two-photon absorption in a photodiode. Appl. Phys. Lett. 2007, 90, 121125. [CrossRef]

125. Schneider, A.; Stillhart, M.; Gunter, P. High efficiency generation and detection of terahertz pulses using laser pulses at telecommunication wavelengths. Opt. Express 2006, 14, 5376-5384. [CrossRef] [PubMed]

126. Cunningham, P.D.; Hayden, L.M. Optical properties of DAST in the THz range. Opt. Express 2010, 18, 23620-23625. [CrossRef] [PubMed]

127. Ohno, S.; Miyamoto, K.; Minamide, H.; Ito, H. New method to determine the refractive index and the absorption coefficient of organic nonlinear crystals in the ultra-wideband THz region. Opt. Express 2010, 18, 17306-17312. [CrossRef] [PubMed]

128. Jazbinsek, M.; Günter, P. Molecular crystals and thin films for photonics. In Handbook of Organic Materials for Electronic and Photonic Devices; Elsevier: Amsterdam, The Netherlands, 2019; pp. 177-210 . [CrossRef]

129. Montemezzani, G.; Alonzo, M.; Coda, V.; Jazbinsek, M.; Guenter, P. Running electric field gratings for detection of coherent radiation. J. Opt. Soc. Am. B-Opt. Phys. 2015, 32, 1078-1083. [CrossRef]

130. Walther, M.; Jensby, K.; Keiding, S.R.; Takahashi, H.; Ito, H. Far-infrared properties of DAST. Opt. Lett. 2000, 25, 911-913. [CrossRef] [PubMed] 
131. Kang, B.J.; Baek, I.H.; Lee, S.H.; Kim, W.T.; Lee, S.J.; Jeong, Y.U.; Kwon, O.P.; Rotermund, F. Highly nonlinear organic crystal OHQ-T for efficient ultra-broadband terahertz wave generation beyond $10 \mathrm{THz}$. Opt. Express 2016, 24, 11054. [CrossRef] [PubMed]

132. Savoini, M.; Huber, L.; Cuppen, H.; Abreu, E.; Kubli, M.; Neugebauer, M.J.; Duan, Y.; Beaud, P.; Xu, J.; Rasing, T.; et al. THz Generation and Detection by Fluorenone Based Organic Crystals. ACS Photonics 2018, 5, 671-677. [CrossRef]

133. Zhang, X.C.; Ma, X.F.; Jin, Y.; Lu, T.M.; Boden, E.P.; Phelps, P.D.; Stewart, K.R.; Yakymyshyn, C.P. Terahertz Optical Rectification From A Nonlinear Organic-Crystal. Appl. Phys. Lett. 1992, 61, 3080-3082. [CrossRef]

134. Dolasinski, B.; Powers, P.E.; Haus, J.W.; Cooney, A. Tunable narrow band difference frequency THz wave generation in DAST via dual seed PPLN OPG. Opt. Express 2015, 23, 3669-3680. [CrossRef] [PubMed]

135. Kawase, K.; Mizuno, M.; Sohma, S.; Takahashi, H.; Taniuchi, T.; Urata, Y.; Wada, S.; Tashiro, H.; Ito, H. Difference-frequency terahertz-wave generation from 4-dimethylamino-N-methyl-4-stilbazolium-tosylate by use of an electronically tuned Ti : sapphire laser. Opt. Lett. 1999, 24, 1065-1067. [CrossRef] [PubMed]

136. Kawase, K.; Hatanaka, T.; Takahashi, H.; Nakamura, K.; Taniuchi, T.; Ito, H. Tunable terahertz-wave generation from DAST crystal by dual signal-wave parametric oscillation of periodically poled lithium niobate. Opt. Lett. 2000, 25, 1714-1716. [CrossRef] [PubMed]

137. Taniuchi, T.; Shikata, J.; Ito, H. Tunable terahertz-wave generation in DAST crystal with dual-wavelength KTP optical parametric oscillator. Electron. Lett. 2000, 36, 1414-1416. [CrossRef]

138. Kawase, K.; Shikata, J.; Ito, H. Narrow-linewidth tunable terahertz-wave sources using nonlinear optics. Solid-State Mid-Infrared Laser Sources 2003, 89, 397-423.

139. Taniuchi, T.; Okada, S.; Nakanishi, H. Widely tunable terahertz-wave generation in an organic crystal and its spectroscopic application. J. Appl. Phys. 2004, 95, 5984-5988. [CrossRef]

140. Taniuchi, T.; Okada, S.; Nakanishi, H. Widely-tunable THz-wave generation in 2-20 THz range from DAST crystal by nonlinear difference frequency mixing. Electron. Lett. 2004, 40, 60-62. [CrossRef]

141. Taniuchi, I.; Adachi, H.; Okada, S.; Sasaki, T.; Nakanishi, H. Continuously tunable THz and far-infrared wave generation from DAST crystal. Electron. Lett. 2004, 40, 549-551. [CrossRef]

142. Taniuchi, T.; Ikeda, S.; Mineno, Y.; Okada, S.; Nakanishi, H. Terahertz properties of a new organic crystal, 4'-dimethylamino-N-methyl-4-stilbazolium p-chlorobenzenesulfonate. Jpn. J. Appl. Phys. Part 2 2005, 44, L932-L934. [CrossRef]

143. Takahashi, Y.; Adachi, H.; Taniuchi, T.; Takagi, M.; Hosokawa, Y.; Onzuka, S.; Brahadeeswaran, S.; Yoshimura, M.; Mori, Y.; Masuhara, H.; et al; Nakanishi, H. Organic nonlinear optical DAST crystals for electro-optic measurement and terahertz wave generation. J. Photochem. Photobiol. A-Chem. 2006, 183, 247-252. [CrossRef]

144. Satoh, T.; Toya, Y.; Yamamoto, S.; Shimura, T.; Kuroda, K.; Takahashi, Y.; Yoshimura, M.; Mori, Y.; Sasaki, T.; Ashihara, S. Generation of mid- to far-infrared ultrashort pulses in 4-dimethylamino-N-methyl-4stilbazolium tosylate crystal. J. Opt. Soc. Am. B-Opt. Phys. 2010, 27, 2507-2511. [CrossRef]

145. Tang, M.; Minamide, H.; Wang, Y.; Notake, T.; Ohno, S.; Ito, H. Tunable terahertz-wave generation from DAST crystal pumped by a monolithic dual-wavelength fiber laser. Opt. Express 2011, 19, 779-786. [CrossRef] [PubMed]

146. Koichi, M.; Miyamoto, K.; Ujita, S.; Saito, T.; Ito, H.; Omatsu, T. Dual-frequency picosecond optical parametric generator pumped by a Nd-doped vanadate bounce laser. Opt. Express 2011, 19, 18523-18528. [CrossRef] [PubMed]

147. Liu, J.; Merkt, F. Generation of tunable Fourier-transform-limited terahertz pulses in $4-N, N$-dimethylamino$4^{\prime}-N^{\prime}$-methyl stilbazolium tosylate crystals. Appl. Phys. Lett. 2008, 93, 131105. [CrossRef]

148. Liu, J.; Schmutz, H.; Merkt, F. Generation of widely tunable Fourier-transform-limited terahertz pulses using narrowband near-infrared laser radiation. J. Mol. Spectrosc. 2009, 256. [CrossRef]

149. Qi, F.; Fan, S.; Notake, T.; Nawata, K.; Matsukawa, T.; Takida, Y.; Minamide, H. An ultra-broadband frequency-domain terahertz measurement system based on frequency conversion via DAST crystal with an optimized phase-matching condition. Laser Phys. Lett. 2014, 11, 085403. [CrossRef]

150. Zhong, K.; Mei, J.; Wang, M.; Liu, P.; Xu, D.; Wang, Y.; Shi, W.; Yao, J.; Teng, B.; Xiao, Y. Compact High-Repetition-Rate Monochromatic Terahertz Source Based on Difference Frequency Generation from a Dual-Wavelength Nd:YAG Laser and DAST Crystal. J. Infrared Millim. Terahertz Waves 2017. [CrossRef] 
151. He, Y.; Wang, Y.; Xu, D.; Nie, M.; Yan, C.; Tang, L.; Shi, J.; Feng, J.; Yan, D.; Liu, H.; et al. High-energy and ultra-wideband tunable terahertz source with DAST crystal via difference frequency generation. Appl. Phys. B-Lasers Opt. 2018, 124, 16. [CrossRef]

152. Uchida, H.; Sugiyama, T.; Suizu, K.; Osumi, T.; Kawase, K. Generation of Widely Tunable Terahertz Waves by Difference-Frequency Generation Using a Configurationally Locked Polyene 2-[3-(4-Hydroxystyryl)-5, 5-Dimethylcyclohex-2-Enylidene] Malononitrile Crystal. Terahertz Sci. Technol. 2011, 4, 132-136.

153. Takayanagi, J.; Kanamori, S.; Suizu, K.; Yamashita, M.; Ouchi, T.; Kasai, S.; Ohtake, H.; Uchida, H.; Nishizawa, N.; Kawase, K. Generation and detection of broadband coherent terahertz radiation using 17-fs ultrashort pulse fiber laser. Opt. Express 2008, 16, 12859-12865. [CrossRef] [PubMed]

154. Katayama, I.; Akai, R.; Bito, M.; Shimosato, H.; Miyamoto, K.; Ito, H.; Ashida, M. Ultrabroadband terahertz generation using $4-N, N$-dimethylamino- $4^{\prime}-N^{\prime}$-methyl-stilbazolium tosylate single crystals. Appl. Phys. Lett. 2010, 97, 021105. [CrossRef]

155. Carey, J.J.; Bailey, R.T.; Pugh, D.; Sherwood, J.N.; Cruickshank, F.R.; Wynne, K. Terahertz pulse generation in an organic crystal by optical rectification and resonant excitation of molecular charge transfer RID B-7993-2008. Appl. Phys. Lett. 2002, 81, 4335-4337. [CrossRef]

156. Kuroyanagi, K.; Yanagi, K.; Sugita, A.; Hashimoto, H.; Takahashi, H.; Aoshima, S.; Tsuchiya, Y. Incident light polarization dependence of terahertz emission spectrum of crystalline $4-N, N$-dimethylamino4'-N'-methyl-stilbazolium tosylate. J. Appl. Phys. 2006, 100, 043117. [CrossRef]

157. Kwon, E.; Okada, S.; Nakanishi, H. Relationship between THz energy decay and molecular vibration of 1-methyl-4-2-[4-(dimethylamino)phenyl]ethenylpyridinium p-toluenesulfonate derivatives. Jpn. J. Appl. Phys. Part 2 2007, 46, L46-L48. [CrossRef]

158. Hauri, C.P.; Ruchert, C.; Vicario, C.; Ardana, F. Strong-field single-cycle THz pulses generated in an organic crystal. Appl. Phys. Lett. 2011, 99, 161116. [CrossRef]

159. Uchida, H.; Oota, K.; Minami, T.; Takeya, K.; Kawase, K. Generation of single-cycle terahertz pulse using Cherenkov phase matching with 4-dimethylamino- $N^{\prime}$-methyl-4'-stilbazolium tosylate crystal. Appl. Phys. Express 2017, 10, 062601. [CrossRef]

160. Vicario, C.; Monoszlai, B.; Hauri, C.P. GV/m Single-Cycle Terahertz Fields from a Laser-Driven Large-Size Partitioned Organic Crystal. Phys. Rev. Lett. 2014, 112. [CrossRef]

161. Chefonov, O.V.; Ovchinnikov, A.V.; Romashevskiy, S.A.; Chai, X.; Ozaki, T.; Savel'ev, A.B.; Agranat, M.B.; Fortov, V.E. Giant self-induced transparency of intense few-cycle terahertz pulses in n-doped silicon. Opt. Lett. 2017, 42, 4889-4892. [CrossRef] [PubMed]

162. Valdivia-Berroeta, G.A.; Heki, L.K.; McMurray, E.A.; Foote, L.A.; Nazari, S.H.; Serafin, L.Y.; Smith, S.J.; Michaelis, D.J.; Johnson, J.A. Alkynyl Pyridinium Crystals for Terahertz Generation. Adv. Opt. Mater. 2018, 6, 1800383. [CrossRef]

163. Shin, M.H.; Lee, S.H.; Kang, B.J.; Jazbinšek, M.; Yoon, W.; Yun, H.; Rotermund, F.; Kwon, O.P. Organic Three-Component Single Crystals with Pseudo-Isomorphic Cocrystallization for Nonlinear Optics and $\mathrm{THz}$ Photonics. Adv. Funct. Mater. 2018, 28, 1805257. [CrossRef]

164. Lu, J.; Lee, S.H.; Li, X.; Lee, S.C.; Han, J.H.; Kown, O.P.; Nelson, K.A. Efficient terahertz generation in highly nonlinear organic crystal HMB-TMS. Opt. Express 2018, 26, 30786. [CrossRef] [PubMed]

165. Lee, S.J.; Kang, B.J.; Shin, M.H.; Lee, S.C.; Lee, S.H.; Jazbinsek, M.; Yun, H.; Kim, D.; Rotermund, F.; Kwon, O.P. Efficient Optical-to-THz Conversion Organic Crystals with Simultaneous Electron Withdrawing and Donating Halogen Substituents. Adv. Opt. Mater. 2018, 6, 1700930. [CrossRef]

166. Lee, S.C.; Kang, B.J.; Lee, J.A.; Lee, S.H.; Jazbinšek, M.; Yoon, W.; Yun, H.; Rotermund, F.; Kwon, O.P. Single Crystals Based on Hydrogen-Bonding Mediated Cation-Anion Assembly with Extremely Large Optical Nonlinearity and Their Application for Intense THz Wave Generation. Adv. Opt. Mater. 2018, 6, 1701258. [CrossRef]

167. Jeong, C.U.; Kang, B.J.; Lee, S.H.; Lee, S.C.; Kim, W.T.; Jazbinsek, M.; Yoon, W.; Yun, H.; Kim, D.; Rotermund, F; et al. Yellow-Colored Electro-Optic Crystals as Intense Terahertz Wave Sources. Adv. Funct. Mater. 2018, 28, 1801143. [CrossRef]

168. Lee, S.C.; Kang, B.J.; Koo, M.J.; Lee, S.H.; Han, J.H.; Choi, J.Y.; Kim, W.T.; Jazbinsek, M.; Yun, H.; Kim, D.; et al. New Electro-Optic Salt Crystals for Efficient Terahertz Wave Generation by Direct Pumping at Ti:Sapphire Wavelength. Adv. Opt. Mater. 2017, 5, 1600758. [CrossRef] 
169. Kim, J.; Lee, S.H.; Lee, S.C.; Jazbinsek, M.; Miyamoto, K.; Omatsu, T.; Lee, Y.S.; Kwon, O.P. Terahertz Phonon Modes of Highly Efficient Electro-optic Phenyltriene OH1 Crystals. J. Phys. Chem. C 2016, 120, 24360-24369. [CrossRef]

170. Vicario, C.; Ovchinnikov, A.V.; Ashitkov, S.I.; Agranat, M.B.; Fortov, V.E.; Hauri, C.P. Generation of 09-mJ THz pulses in DSTMS pumped by a Cr:Mg_2SiO_4 laser. Opt. Lett. 2014, 39, 6632. [CrossRef] [PubMed]

171. Agranat, M.; Chefonov, O.; Ovchinnikov, A.; Ashitkov, S.; Fortov, V.; Kondratenko, P. Damage in a Thin Metal Film by High-Power Terahertz Radiation. Phys. Rev. Lett. 2018, 120. [CrossRef] [PubMed]

172. Lee, S.H.; Kang, B.J.; Yoo, B.W.; Lee, S.C.; Lee, S.J.; Jazbinsek, M.; Yun, H.; Rotermund, F.; Kwon, O.P. Terahertz Phonon Mode Engineering of Highly Efficient Organic Terahertz Generators. Adv. Funct. Mater. 2017, 27, 1605583. [CrossRef]

173. Puc, U.; Bach, T.; Krajewski, M.; Medrano, C.; Jazbinsek, M. Ultrabroadband terahertz time domain spectroscopy based on organic crystals. In Proceedings of the 8th International Workshop on Terahertz Technology and Applications, Kaiserslautern, Germany, 20-21 March 2018; Book of Abstracts.

174. Abina, A.; Puc, U.; Jeglič, A.; Prah, J.; Venckevičius, R.; Kašalynas, I.; Valušis, G.; Zidanšek, A. Qualitative and quantitative analysis of calcium-based microfillers using terahertz spectroscopy and imaging. Talanta 2015, 143, 169-177. [CrossRef] [PubMed]

175. Abina, A.; Puc, U.; Jeglič, A.; Zidanšek, A. Structural characterization of thermal building insulation materials using terahertz spectroscopy and terahertz pulsed imaging. NDT E Int. 2016, 77, 11-18. [CrossRef]

176. Abina, A.; Puc, U.; Jeglič, A.; Zidanšek, A. Structural analysis of insulating polymer foams with terahertz spectroscopy and imaging. Polym. Test. 2013, 32, 739-747. [CrossRef]

177. Lavrič, Z.; Pirnat, J.; Lužnik, J.; Puc, U.; Trontelj, Z.; Srčič, S. 14 N Nuclear Quadrupole Resonance Study of Piroxicam: Confirmation of New Polymorphic Form V. J. Pharm. Sci. 2015, 104, 1909-1918. [CrossRef] [PubMed]

178. Puc, U.; Abina, A.; Jeglič, A.; Zidanšek, A.; Kašalynas, I.; Venckevičius, R.; Valušis, G. Spectroscopic Analysis of Melatonin in the Terahertz Frequency Range. Sensors 2018, 18, 4098. [CrossRef] [PubMed]

179. Yin, M.; Tang, S.; Tong, M. Identification of edible oils using terahertz spectroscopy combined with genetic algorithm and partial least squares discriminant analysis. Anal. Methods 2016, 8, 2794-2798. [CrossRef]

180. Woldegeorgis, A.; Kurihara, T.; Beleites, B.; Bossert, J.; Grosse, R.; Paulus, G.G.; Ronneberger, F.; Gopal, A. $\mathrm{THz}$ Induced Nonlinear Effects in Materials at Intensities above $26 \mathrm{GW} / \mathrm{cm} 2$. J. Infrared Millim. Terahz Waves 2018, 39, 667-680. [CrossRef]

181. Nicoletti, D.; Cavalleri, A. Nonlinear light-matter interaction at terahertz frequencies. Adv. Opt. Photonics 2016, 8, 401. [CrossRef]

182. Hirori, H.; Doi, A.; Blanchard, F.; Tanaka, K. Single-cycle terahertz pulses with amplitudes exceeding $1 \mathrm{MV} / \mathrm{cm}$ generated by optical rectification in $\mathrm{LiNbO}_{3}$. Appl. Phys. Lett. 2011, 98, 091106. [CrossRef]

183. Yoshioka, K.; Minami, Y.; Shudo, K.I.; Dao, T.D.; Nagao, T.; Kitajima, M.; Takeda, J.; Katayama, I. Terahertz-Field-Induced Nonlinear Electron Delocalization in Au Nanostructures. Nano Lett. 2015, 15, 1036-1040. [CrossRef] [PubMed]

184. Tarekegne, A.T.; Iwaszczuk, K.; Zalkovskij, M.; Strikwerda, A.C.; Jepsen, P.U. Impact ionization in high resistivity silicon induced by an intense terahertz field enhanced by an antenna array. New J. Phys. 2015, 17, 043002. [CrossRef]

185. Blanchard, F.; Ropagnol, X.; Hafez, H.; Razavipour, H.; Bolduc, M.; Morandotti, R.; Ozaki, T.; Cooke, D.G. Effect of extreme pump pulse reshaping on intense terahertz emission in lithium niobate at multimilliJoule pump energies. Opt. Lett. 2014, 39, 4333. [CrossRef] [PubMed]

186. Dienst, A.; Hoffmann, M.C.; Fausti, D.; Petersen, J.C.; Pyon, S.; Takayama, T.; Takagi, H.; Cavalleri, A. Bi-directional ultrafast electric-field gating of interlayer charge transport in a cuprate superconductor. Nat. Photonics 2011, 5, 485-488. [CrossRef]

187. Allodi, M.A.; Finneran, I.A.; Blake, G.A. Nonlinear terahertz coherent excitation of vibrational modes of liquids. J. Chem. Phys. 2015, 143, 234204. [CrossRef] [PubMed]

188. Lu, J.; Li, X.; Zhang, Y.; Hwang, H.Y.; Ofori-Okai, B.K.; Nelson, K.A. Two-Dimensional Spectroscopy at Terahertz Frequencies. Top. Curr. Chem. 2018, 376. [CrossRef] [PubMed]

189. Shalaby, M.; Hauri, C.P. Air nonlinear dynamics initiated by ultra-intense lambda-cubic terahertz pulses. Appl. Phys. Lett. 2015, 106, 181108. [CrossRef] 
190. Shalaby, M.; Vicario, C.; Hauri, C.P. High-performing nonlinear visualization of terahertz radiation on a silicon charge-coupled device. Nat. Commun. 2015, 6, 8439. [CrossRef] [PubMed]

191. Shalaby, M.; Vicario, C.; Hauri, C.P. Low frequency terahertz-induced demagnetization in ferromagnetic nickel. Appl. Phys. Lett. 2016, 108, 182903. [CrossRef]

192. Shalaby, M.; Vicario, C.; Hauri, C.P. Simultaneous electronic and the magnetic excitation of a ferromagnet by intense THz pulses. New J. Phys. 2016, 18, 013019. [CrossRef]

193. Shalaby, M.; Vicario, C.; Hauri, C.P. Extreme nonlinear terahertz electro-optics in diamond for ultrafast pulse switching. APL Photonics 2017, 2, 036106. [CrossRef]

194. Vicario, C.; Shalaby, M.; Hauri, C.P. Subcycle Extreme Nonlinearities in GaP Induced by an Ultrastrong Terahertz Field. Phys. Rev. Lett. 2017, 118. [CrossRef] [PubMed]

195. V. Chefonov, O.; V. Ovchinnikov, A.; A. Evlashin, S.; B. Agranat, M. Damage Threshold of Ni Thin Film by Terahertz Pulses. J. Infrared Millim. Terahz Waves 2018, 39, 1047-1054. [CrossRef]

196. Fan, S.; Qi, F.; Notake, T.; Nawata, K.; Matsukawa, T.; Takida, Y.; Minamide, H. Real-time terahertz wave imaging by nonlinear optical frequency up-conversion in a 4-dimethylamino- $\mathrm{N}^{\prime}$-methyl-4'-stilbazolium tosylate crystal. Appl. Phys. Lett. 2014, 104, 101106. [CrossRef]

197. Bezhanov, S.G.; Uryupin, S.A. Nonlinear transmission and reflection of a strong terahertz pulse by a metal film. Opt. Lett. 2018, 43, 3069-3072. [CrossRef] [PubMed]

198. Zhong, S. Progress in terahertz nondestructive testing: A review. Front. Mech. Eng. 2018. [CrossRef]

199. Kemp, M.C. Explosives Detection by Terahertz Spectroscopy-A Bridge Too Far? IEEE Trans. Terahertz Sci. Technol. 2011, 1, 282-292. [CrossRef]

200. Haddad, J.E.; Bousquet, B.; Canioni, L.; Mounaix, P. Review in terahertz spectral analysis. Trends Anal. Chem. 2013, 44, 98-105. [CrossRef]

201. Shen, Y.C.; Taday, P.F.; Newnham, D.A.; Pepper, M. Chemical mapping using reflection terahertz pulsed imaging. Semicond. Sci. Technol. 2005, 20, S254-S257. [CrossRef]

202. Charron, D.M.; Ajito, K.; Kim, J.Y.; Ueno, Y. Chemical Mapping of Pharmaceutical Cocrystals Using Terahertz Spectroscopic Imaging. Anal. Chem. 2013, 85, 1980-1984. [CrossRef] [PubMed]

203. Majkić, A.; Puc, U.; Franke, A.; Kirste, R.; Collazo, R.; Sitar, Z.; Zgonik, M. Optical properties of aluminum nitride single crystals in the THz region. Opt. Mater. Express 2015, 5, 2106-2111. [CrossRef]

204. Kawase, K.; Shibuya, T.; Hayashi, S.; Suizu, K. THz imaging techniques for nondestructive inspections. Comptes Rendus Phys. 2010, 11, 510-518. [CrossRef]

205. Ajito, K.; Ueno, Y.; Song, H.J.; Tamechika, E.; Kukutsu, N. Terahertz Spectroscopic Imaging of Polymorphic Forms in Pharmaceutical Crystals. Mol. Cryst. Liq. Cryst. 2011, 538, 33-38. [CrossRef]

206. Liu, H.B.; Chen, Y.; Zhang, X.C. Characterization of Anhydrous and Hydrated Pharmaceutical Materials with THz Time-Domain Spectroscopy. J. Pharm. Sci. 2007, 96, 927-934. [CrossRef] [PubMed]

207. Hisazumi, J.; Suzuki, T.; Wakiyama, N.; Nakagami, H.; Terada, K. Chemical Mapping of Hydration and Dehydration Process of Theophylline in Tablets Using Terahertz Pulsed Imaging. Chem. Pharm. Bull. 2012, 60, 831-836. [CrossRef] [PubMed]

208. Lin, H.; Dong, Y.; Markl, D.; Williams, B.M.; Zheng, Y.; Shen, Y.; Zeitler, J.A. Measurement of the Intertablet Coating Uniformity of a Pharmaceutical Pan Coating Process with Combined Terahertz and Optical Coherence Tomography In-Line Sensing. J. Pharm. Sci. 2017, 106, 1075-1084. [CrossRef] [PubMed]

209. May, R.K.; Su, K.; Han, L.; Zhong, S.; Elliott, J.A.; Gladden, L.F.; Evans, M.; Shen, Y.; Zeitler, J.A. Hardness and Density Distributions of Pharmaceutical Tablets Measured by Terahertz Pulsed Imaging. J. Pharm. Sci. 2013, 102, 2179-2186. [CrossRef] [PubMed]

210. Watanabe, Y.; Kawase, K.; Ikari, T.; Ito, H.; Ishikawa, Y.; Minamide, H. Component spatial pattern analysis of chemicals using terahertz spectroscopic imaging. Appl. Phys. Lett. 2003, 83, 800-802. [CrossRef]

211. Sasaki, T.; Sakamoto, T.; Otsuka, M. Detection of Impurities in Organic Crystals by High-Accuracy Terahertz Absorption Spectroscopy. Anal. Chem. 2018, 90, 1677-1682. [CrossRef] [PubMed]

212. Puc, U.; Abina, A.; Jeglič, A.; Zidanšek, A. Terahertz spectral characterisation of active substance in commercial pharmaceutical tablets. In Proceedings of Abstracts; CAMTP: Maribor, Slovenia, 2012.

213. Boyjoo, Y.; Pareek, V.K.; Liu, J. Synthesis of micro and nano-sized calcium carbonate particles and their applications. J. Mater. Chem. A 2014, 2, 14270-14288. [CrossRef] 
214. Puc, U.; Abina, A.; Rutar, M.; Zidanšek, A.; Jeglič, A.; Valušis, G. Terahertz spectroscopic identification of explosive and drug simulants concealed by various hiding techniques. Appl. Opt. 2015, 54, 4495-4502. [CrossRef] [PubMed]

215. Beigang, R.; Biedron, S.G.; Dyjak, S.; Ellrich, F.; Haakestad, M.W.; Hübsch, D.; Kartaloglu, T.; Ozbay, E.; Ospald, F.; Palka, N.; et al. Comparison of terahertz technologies for detection and identification of explosives. In Terahertz Physics, Devices, and Systems VIII: Advanced Applications in Industry and Defense; Proc. SPIE 2014, 9102, 91020C . [CrossRef]

216. Qin, J.; Ying, Y.; Xie, L. The Detection of Agricultural Products and Food Using Terahertz Spectroscopy: A Review. Appl. Spectrosc. Rev. 2013, 48, 439-457. [CrossRef]

217. Abina, A.; Puc, U.; Jeglič, A.; Zidanšek, A. Applications of Terahertz Spectroscopy in the Field of Construction and Building Materials. Appl. Spectrosc. Rev. 2015, 50, 279-303. [CrossRef]

218. Abina, A.; Puc, U.; Jeglič, A.; Zidanšek, A. Applications of Terahertz Spectroscopy and Imaging for Building Materials Characterization. In Conference Proceedings MIDEM 2015; Society for Microelectronics, Electronic Components and Materials: Bled, Slovenia, 2015.

219. Sun, Q.; He, Y.; Liu, K.; Fan, S.; Parrott, E.P.J.; Pickwell-MacPherson, E. Recent advances in terahertz technology for biomedical applications. Quant. Imaging Med. Surg. 2017, 7, 345-355. [CrossRef] [PubMed]

220. Graber, B.; Kim, C.; Wu, D.H. High SNR single measurements of trace gas phase spectra at THz frequencies. Appl. Phys. Lett. 2017, 111. [CrossRef]

221. Guillet, J.P.; Roux, M.; Wang, K.; Ma, X.; Fauquet, F.; Balacey, H.; Recur, B.; Darracq, F.; Mounaix, P. Art Painting Diagnostic Before Restoration with Terahertz and Millimeter Waves. J. Infrared Millim. Terahz Waves 2017, 38, 369-379. [CrossRef]

222. Reid, M.E.; Hartley, I.D.; Todoruk, T.M. 19-Terahertz applications in the wood products industry. In Handbook of Terahertz Technology for Imaging, Sensing and Communications; Saeedkia, D., Ed.; Woodhead Publishing Series in Electronic and Optical Materials; Woodhead Publishing: Cambridge, UK, 2013; pp. 547-578. [CrossRef]

(C) 2019 by the authors. Licensee MDPI, Basel, Switzerland. This article is an open access article distributed under the terms and conditions of the Creative Commons Attribution (CC BY) license (http:/ / creativecommons.org/licenses/by/4.0/). 